\title{
CONFINED MIXING OF MULTIPLE TRANSVERSE JETS
}

\author{
A Thesis \\ presented to \\ the Faculty of California Polytechnic State University, \\ San Luis Obispo
}

\author{
In Partial Fulfillment \\ of the Requirements for the Degree \\ Master of Science in Aerospace Engineering
}

by

Allen J. Bishop
December 2012 
(C) 2012

Allen J. Bishop

ALL RIGHTS RESERVED 


\section{COMMITTEE MEMBERSHIP}

TITLE:

Confined Mixing of Multiple Transverse Jets

AUTHOR:

Allen J. Bishop

DATE SUBMITTED:

December 2012

COMMITTEE CHAIR:

Dr. Dianne DeTurris, Professor

COMMITTEE MEMBER: Dr. Jin Tso, Professor

COMMITTEE MEMBER: Dr. Kim Shollenberger, Professor

COMMITTEE MEMBER: $\quad$ Dr. David Forliti, Principal Investigator, Project Themis 


\section{ABSTRACT \\ Confined Mixing of Multiple Transverse Jets}

Allen J. Bishop

The mixing performance of multiple transverse jets has been evaluated experimentally.

Measurement techniques included laser Doppler velocimetry and planar laser induced fluorescence. Basic findings are consistent with results presented in literature for single jet mixing behavior. Mixing performance has been compared to literature for the single jet case and the Holdeman parameter has been re-evaluated for effectiveness at low jet numbers. A single jet in a confined crossflow was found to have a local minimum at $B(d / D)=0.721$. Results for two jets indicate monotonically decreasing unmixedness for the range of conditions tested, with no local optimum apparent. Data for three jets indicate a local optimum at $B(d / D)=0.87$ and relatively flat range of mixing performance in the range of $0.75<$ $B(d / D)<1.25$. Six jets indicate a minimum unmixedness near $B(d / D)=0.5$, but exhibited poorer mixing performance than all other configurations at the highest values of $B(d / D)$ tested. The most optimum configuration tested was six jets at $B(d / D)=0.5$, resulting in an unmixedness of 0.0192 . This value was $76 \%$ lower than the next lowest configuration (three jets) at the same $B(d / D)$. Total momentum was found to collapse the data well, as configurations more closely matched a historical correlation for second moment of a single confined jet more closely.

Keywords: jet-in-crossflow, transverse jet, confined jet, mixing performance, unmixedness, multiple confined transverse jets, planar laser induced fluorescence 


\section{ACKNOWLEDGMENTS}

I would like to offer my sincerest thanks to all the great people at the Air Force Research Laboratory. Without them, this thesis would not be possible. Most notably, I would like to thank Dr. Richard Cohn, Dr. David Forliti, Mr. Nils Sedano, Mr. Robert Bernstein, Mr. John Stephens, Dr. Farhad Davoudzadeh, Dr. Alex Schumaker and countless others. Your guidance and patience were essential to my success.

I would also like to thank my thesis committee at Cal Poly. Drs. Dianne DeTurris, Jin Tso and Kim Shollenberger have offered their support and criticism to ensure that my contributions to fluid mechanics are as relevant as possible.

Finally, I would like to thank my family, whose unending patience and support have allowed me to reach the capstone of my education. I offer my sincerest thanks to all of you. 


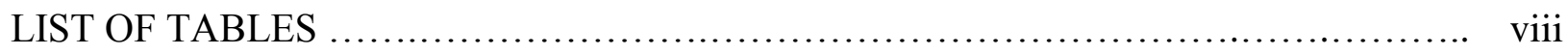

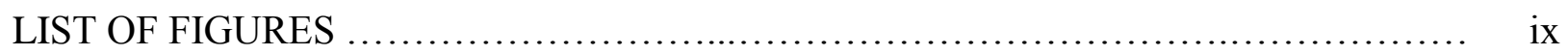

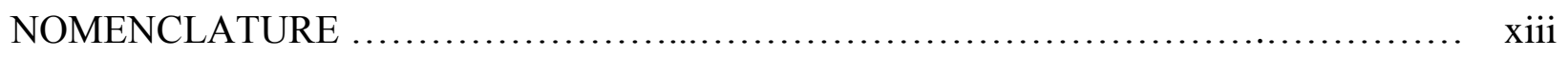

1. INTRODUCTION ......................................................... 1

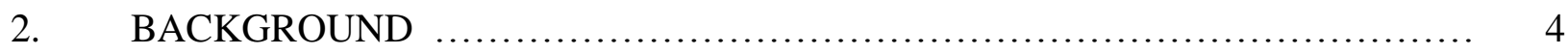

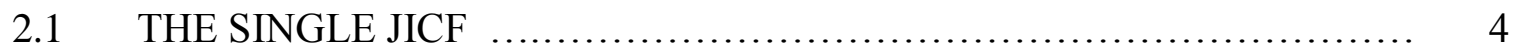

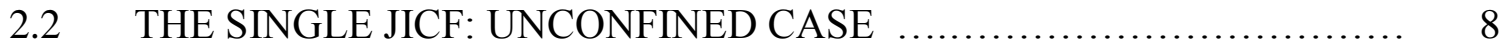

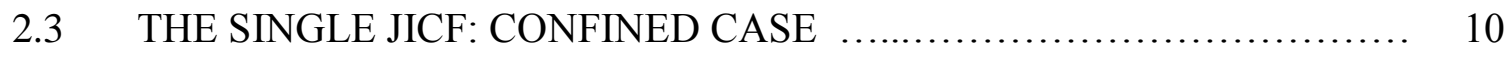

$2.4 \quad$ MULTIPLE CONFINED JICF _...................................... 13

2.5 MEASUREMENT TECHNIQUES _.................................. 21

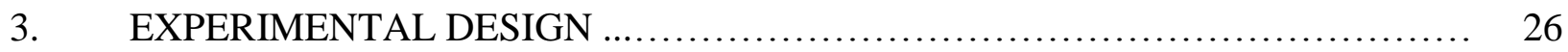

$3.1 \quad$ HISTORICAL CONSIDERATIONS ................................ 26

$3.2 \quad$ APPARATUS CONSIDERATIONS ................................ 30

3.3 OPERATING LIMITS AND TEST POINTS ............................. 32

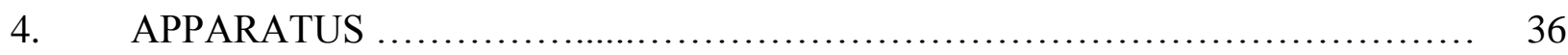

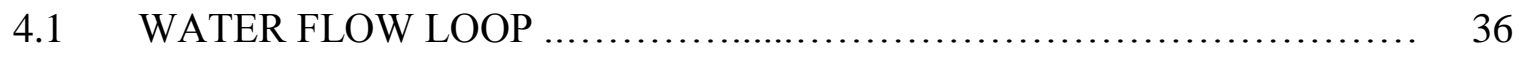

4.1.1 PRIMARY FLOW LOOP ........................................ 36

4.1.2 INJECTION SYSTEM .......................................... 38

4.1.3 TEST SECTION …............................................... 41

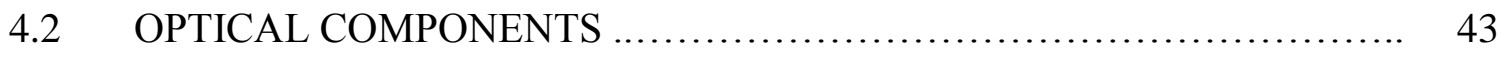

5. VALIDATIONAND CALIBRATION .................................... 44

$5.1 \quad$ VALIDATION OF ANALYSIS CODE ................................ 44

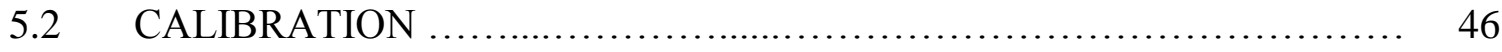

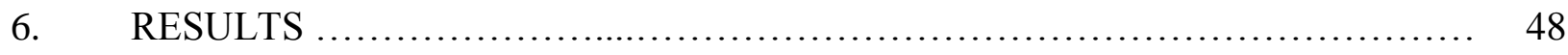


6.1 MIXING PERFORMANCE AS A FUNCTION OF $B(d / D) \ldots \ldots \ldots \ldots \ldots . . \ldots 48$

6.2 MIXING PERFORMANCE AS A FUNCTION OF NUMBER OF JETS ... 52

6.3 MIXING PERFORMANCE AS A FUNCTION OF MOMENTUM RATIO 54

$6.4 \quad$ SUMMARY PLOTS .................................................... 56

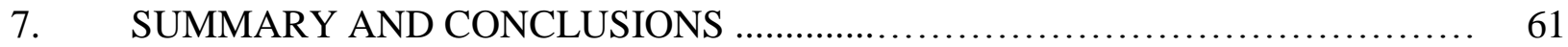

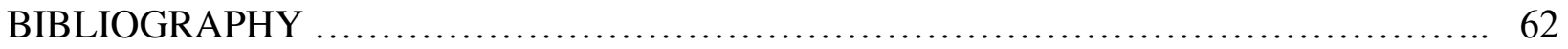

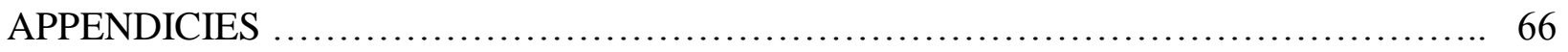

A. LASER DOPPLER VELOCIMETRY .................................. 66

B. DERIVATION OF EXPECTED HOLDEMAN SCALING DEVIATION ... 69

C. COMPLETE SCHEMATIC FOR FLOW LOOP FACILITY ............... 71

D. UNCERTAINTY ANALYSIS ...................................... 73 


\section{LIST OF TABLES}

Table Page

1. Current US launch vehicle propulsion systems $\quad \ldots \ldots \ldots \ldots \ldots \ldots \ldots \ldots \ldots \ldots \ldots \ldots \ldots$

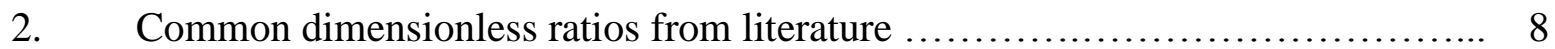

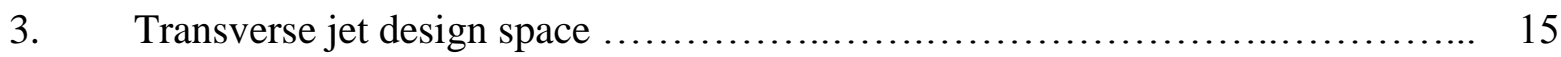




\section{LIST OF FIGURES}

Figure

Page

1. Anatomy of the transverse jet ............................................ 5

2. Evolution of shear layer wrap-up into CVP ................................ 6

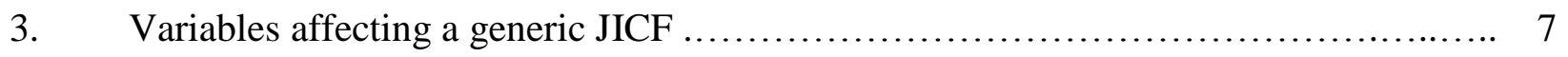

4. Second moment for single jet injection $(d / D=0.071) \ldots \ldots \ldots \ldots \ldots \ldots \ldots \ldots \ldots \ldots \ldots \ldots$

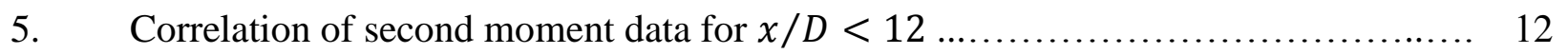

6. Two-dimensional duct configuration for evaluating multiple JICF mixing ........... 14

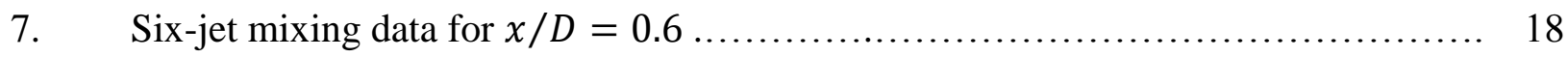

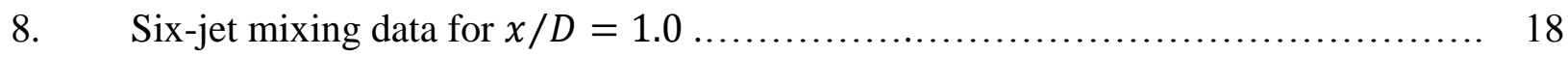

9. Mixing as a function of the number of jets and momentum flux ratio at $x / D=0.6 \quad 19$

10. Mixing as a function of the number of jets and momentum flux ratio at $x / D=1.0 \quad 19$

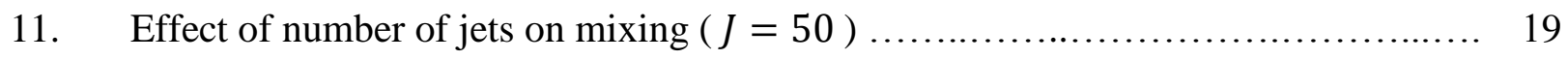

12. Mixing non-uniformity for $n=8$ configuration at $x / D=1.2 \ldots \ldots \ldots \ldots \ldots \ldots \ldots \ldots . . . \ldots 1$

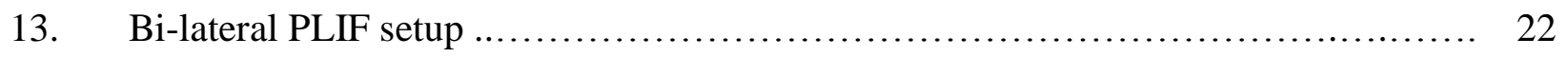

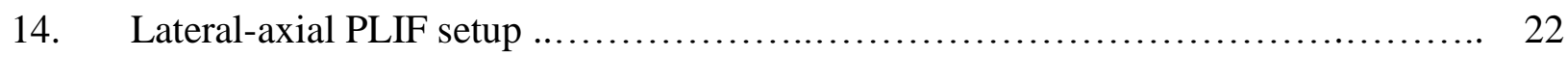

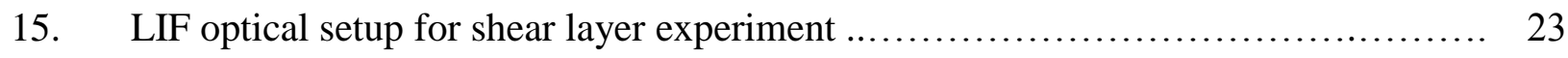


16. Fluorescence intensity as a function of concentration

17. Fluorescence intensity as a function of fluid $\mathrm{pH}$

18. Blowing ratio versus number of jets for historical data

19. Diameter ratio versus number of jets for historical data

20. Cut-away of test section concept

21. Test section concept with jet fluid manifold

22. Linear distribution injection manifold

23. Design space for $=1$

24. Design space for $n=2$

25. Design space for $n=3$

26. Design space for $=6$

27. Flow loop schematic 36

28. Primary flow loop run tank and pump 37

29. Bypass and upstream piping 38

30. Orifice plate, calibration feed line and turbulent run-up ...

31. Jet fluid injection tanks 39

32. Injection manifold and hardware 
33. Primary flow loop piping and injection block

34. Close-up of jet insert $\mathrm{B}(d / D=0.165)$

35. Test section with optical equipment

36. Test section outflow piping

37. Optical shaping components 43

38. Radial step function used to validate image analysis code

39. Convergence of code output parameters using test distribution 45

40. Analysis code output error as a function of image size 46

41. $\mathrm{R}^{2}$ values for calibration data used on one and two jet data 47

42. $\mathrm{R}^{2}$ values for calibration data used on three jets 47

43. $\mathrm{R}^{2}$ values for calibration data used on six jets

44. Unmixedness as a function of $(d / D)$ 49

45. Jet mass fraction for $n=1, B(d / D)=0.749$ 49

46. Jet mass fraction for $n=1, B(d / D)=0.983$

47. Jet mass fraction for $n=1, B(d / D)=1.221$

48. Jet mass fraction for $n=1, B(d / D)=1.468$ 
49. Unmixedness of six jet configurations at various $\mathrm{x} / \mathrm{D}$

50. Calculated optimum momentum flux ratio based upon relative unmixedness

51. Unmixedness as a function of the number of jets

52. Location-adjusted second moment of concentration versus momentum ratio

53. Location-adjusted second moment of concentration versus total momentum ratio

54. Summary plot for single jet configuration

55. Summary plot for two jet configuration

56. Summary plot for three jet configuration

57. Summary plot for six jet configuration

A1. Interference pattern of an LDV setup

A2. Normalized velocity profile for $Q=1.61 \mathrm{GPM}$

A3. Normalized velocity profile for $Q=2.34 \mathrm{GPM}$

A4. Normalized velocity profile for $Q=8.30 \mathrm{GPM}$

A5. Velocity normalization at a sudden axial contraction

B1. Comparative cases for jet injection

B2. Jet distribution within a pipe

C1. Complete piping schematic for flow facility 


\section{NOMENCLATURE}

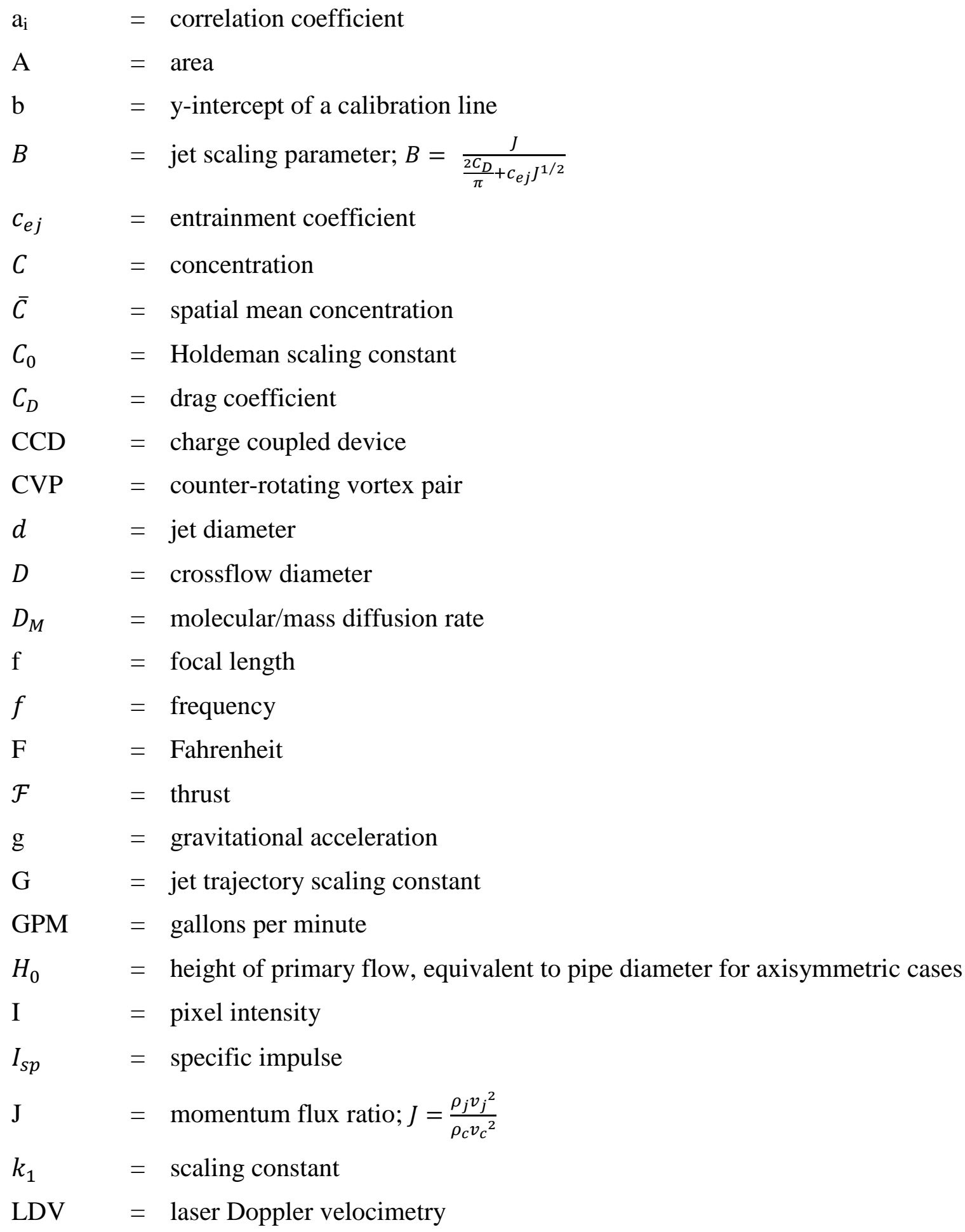




$$
\begin{array}{ll}
\text { LIF } & =\text { laser-induced fluorescence } \\
\mathrm{LSR} & =\text { least scale reading } \\
\mathrm{m} & =\text { slope of a calibration line } \\
\dot{m} & =\text { mass flow rate } \\
M & =\text { second moment of a given variable } \\
M_{0} & =\text { value of second moment at injection location } \\
n & =\text { number of jets } \\
\mathrm{N} & =\text { number of samples } \\
\mathrm{psi} & =\text { pounds per square inch } \\
\mathrm{P} & =\text { pressure } \\
\mathrm{PLIF} & =\text { planar laser-induced fluorescence } \\
Q & =\text { volumetric flow rate } \\
r & =\text { blowing ratio; } r=\sqrt{\frac{\rho_{j} v_{j}^{2}}{\rho_{c} v_{c}^{2}}} \\
\mathrm{rpm} & =\text { revolutions per minute } \\
R_{1 / 2} & =\text { radius which splits the crossflow area into equal areas } \\
\mathrm{S} & =\text { orifice spacing } \\
S c & =\text { Schmidt number; } S c=\frac{\mu}{\rho D_{M}} \\
T & =\text { temperature } \\
\bar{T} & =\text { spatial mean temperature } \\
\mathrm{U} & =\text { unmixedness; } \\
v & =\text { velocity } \\
\mathrm{V} & =\text { voltage } \\
x & =\text { axial distance from jet injection center } \\
y, Y & =\text { vertical distance from jet injection center } \\
z, Z & =\text { normal distance from jet injection center } \\
\bar{Z} & =\text { mean mixture fraction as a function of relative flow rates; } \bar{Z}=\frac{n Q_{j}}{n Q_{j}+Q_{c}} \\
& =\text { momentum ratio; } \beta=\frac{\rho_{j} v_{j}^{2} d^{2}}{\rho_{c} v_{c}^{2} D^{2}} \\
& =
\end{array}
$$




$\begin{array}{ll}\lambda & =\text { wavelength } \\ \mu & =\text { viscosity } \\ \rho & =\text { density } \\ \sigma & =\text { standard deviation }\end{array}$

Subscripts:

$\begin{array}{ll}\operatorname{avg} & =\text { mean average } \\ \mathrm{c} & =\text { crossflow } \\ \mathrm{eq} & =\text { equilibrium } \\ \mathrm{i} & =\text { local value } \\ \text { in } & =\text { inlet } \\ \mathrm{j} & =\text { jet } \\ \text { rms } & =\text { root mean square }\end{array}$




\section{Introduction}

Liquid-propellant rocket engines have been the standard for several generations of launch vehicles from the Saturn family to today's Atlas, Delta and Falcon vehicles. However, these vehicles have all been designed to the mold of Evolved Expendable Launch Vehicles (EELV) with the goal of making launches more affordable via universalizing integration operations for fueling and launch platforms. Development of reusable propulsion systems had also been prohibitively expensive (with one exception being the Space Shuttle Main Engine).

In recent years the Department of Defense and NASA have vested renewed interest in reusable systems, as long-term budgets have tightened in the last decade and will continue to do so. This sparked the Integrated High Payoff Rocket Propulsion Technology (IHPRPT) program, primarily under the direction of Air Force Research Laboratory. The goal of this program is to convene the designers and users of rocket propulsion technology to direct design and production of next generation systems in a realistic and cost-effective manner.

Modern full-scale liquid rocket engines utilize one of two fuels. The first is Rocket Propellant 1 (RP-1) which is a highly refined grade of kerosene. The second is cryogenic liquid hydrogen (LH2). Either of these is then combined with cryogenic liquid oxygen (LOX) and combusted. The effectiveness of a rocket depends highly on its mission, making it difficult to directly compare one launch system to another. This discussion focuses only on the first-stage boosters of the respective systems.

One metric that can be used to compare systems directly is their specific impulse, commonly referred to as $I_{\text {sp }}$ and given by equation 1 as

$$
I_{s p}=\frac{\mathcal{F}}{m g}
$$


where $\mathcal{F}$ is the thrust produced, $\dot{m}$ is the total propellant mass flow rate and $\mathrm{g}$ is the acceleration due to gravity. Specific impulse may be thought of as fuel efficiency for a rocket engine, i.e. how efficiently it produces thrust per unit mass.

The Atlas V first stage uses a single Russian-built RD-180 engine, outputting 860 klbf of thrust with an $\mathrm{I}_{\mathrm{sp}}$ of 311 seconds [1]. Its primary competitor, the Delta IV, uses one RS-68 (U.S., Rocketdyne) outputting 744 klbf of thrust and an $\mathrm{I}_{\mathrm{sp}}$ of 410 seconds [1]. The newest entry to the US launch vehicle market is the Falcon 9, which uses nine Merlin 1D engines each outputting 147 klbf of thrust at an $\mathrm{I}_{\mathrm{sp}}$ of 282 seconds [2]. Table 1 summarizes the current state of the art.

\begin{tabular}{|l|c|c|c|}
\hline Vehicle & Atlas V $^{\mathbf{1}}$ & Delta IV $^{\mathbf{1}}$ & Falcon 9 $^{\mathbf{2}}$ \\
\hline Engine Configuration & One RD-180 & One RS-68 & Nine Merlin 1C \\
\hline Propellants & RP-1/LOX & LH2/LOX & RP-1/LOX \\
\hline Total Thrust (sea level static) & $866,200 \mathrm{lbf}$ & $663,000 \mathrm{lbf}$ & $1,323,000 \mathrm{lbf}$ \\
\hline $\mathrm{I}_{\text {sp }}$ (sea level static) & $312.7 \mathrm{sec}$ & $359 \mathrm{sec}$ & $311 \mathrm{sec}$ \\
\hline Engine Thrust-to-Weight Ratio & 71.2 & 44.6 & 150 \\
\hline
\end{tabular}

Table 1: Current US launch vehicle propulsion systems

One of the challenges associated with the program is the development of the next generation firststage booster system. At low altitudes, thrust is more important to overall system performance than $\mathrm{I}_{\mathrm{sp}}$. However, a designer would always choose the highest $\mathrm{I}_{\mathrm{sp}}$ available within design constraints.

Additionally, RP fuels are preferable to hydrogen first-stage boosters since they require less thermal insulation and are more volumetrically energy-dense. Another important consideration is the engine thrust-to-weight ratio. This metric is important because weight savings in the engine for the same level of thrust allow for increase in the allowable payload weight. Although the RD-180 and Merlin 1C are better than the RS-68 in this respect, their designs have room for improvement with new technologies.

In order to extend the usable life of an engine, typical failure points need to be addressed. One of these failure points is the turbine. A combustion device produces a high enthalpy gas that drives the turbine, consequently driving a shaft attached to a pump for a propellant. Since the turbine is exposed to extreme environments in excess of $1000^{\circ} \mathrm{F}$, it is a common source of failure for all kinds of engines, including aircraft turbojets and industrial gas turbines. When the turbine is exposed to variations in 
conditions (inlet pressure, heat fluxes, density or species) it contributes to low- and high-cycle fatigue, limiting the overall life of the turbine before failure (cracking or blade separation). In order to meet life requirements, high spatial and temporal uniformity is required at the turbine inlet. In order to predict the inlet conditions for the turbine, adequate study must be given to the mixing of propellants introduced in the hot gas source. One method to accomplish this is the jet-in-crossflow, also known as a transverse jet. The motivation for the current effort is to determine mixing behavior in a geometry simulating a combustion device in order to minimize scalar variations seen by the turbine. The variables have been selected by reviewing background literature and identifying the most critical independent parameters that contribute to jet mixing. These include jet diameter ratio, number of jets and momentum flux ratio. An experimental apparatus has been constructed to vary each parameter independently and record appropriate pressures and flow rates. Mixing has been quantified using an optical measurement technique and calculated for the current design space and presented as a function of the design variables. Finally, recommendations have been made with regards to experimental design for future experiments. 


\section{Background}

The jet-in-crossflow (also known as transverse jet or JICF) is one of the most fundamental and elegant three-dimensional flowfields. The JICF represents a configuration that introduces one fluid perpendicular to a bulk fluid for the purpose of mixing the two flows and occurs in both nature and engineering. The applications associated with the JICF are numerous, ranging from thrust vectoring of VSTOL aircraft, dispersion of pollutants from smokestacks, tee mixers for the chemical engineering industry, gas turbine burners in aircraft engines, film cooling and flame holding for generalized combustion devices. Transverse jets have therefore received a substantial amount of research attention for the unconfined case (a single jet issuing into a nominally-infinite bulk crossflow). Single confined transverse jets have also been studied extensively, with most of the efforts focusing on jet trajectory rather than bulk mixing. Studies of multiple confined transverse jets have been limited to a higher number of jets (eight to sixteen or more) as they play more of a role with gas turbine burners. There is consequently a gap in the literature for two to eight jets which constrains the design space for propulsion mixing devices. It is therefore the goal of the current effort to eliminate the gap and provide enhanced flexibility for future designs.

\section{$2.1 \quad$ The Single JICF}

Figure 1, originally developed by Fric and Roshko [3], details the macro-level flowfield features of the JICF. The most comprehensively studied flow feature is the counter-rotating vortex pair (CVP). This is due to the fact that it is credited as the method by which the jet fluid entrains crossflow fluid, absorbs its streamwise momentum and adjusts the jet trajectory. This relationship is true for the far field mixing (x/D >> 1) and also in the near field for jets of a low velocity ratio. The CVP maintains its structure for up to hundreds of jet diameters in the streamwise direction if otherwise unperturbed. The CVP has also been named the "kidney" vortex, as the shape of the vortex resembles a kidney when viewed in the plane perpendicular to the jet trajectory as illustrated in Fig. 2. The mechanism by which the CVP forms has been a subject of debate over years of research. According to Hasselbrink and Mungal [4], the shear layer 
between the jet and the crossflow becomes distorted and reoriented, "wrapping up" into the macro structure of the CVP and can be explained by momentum conservation.

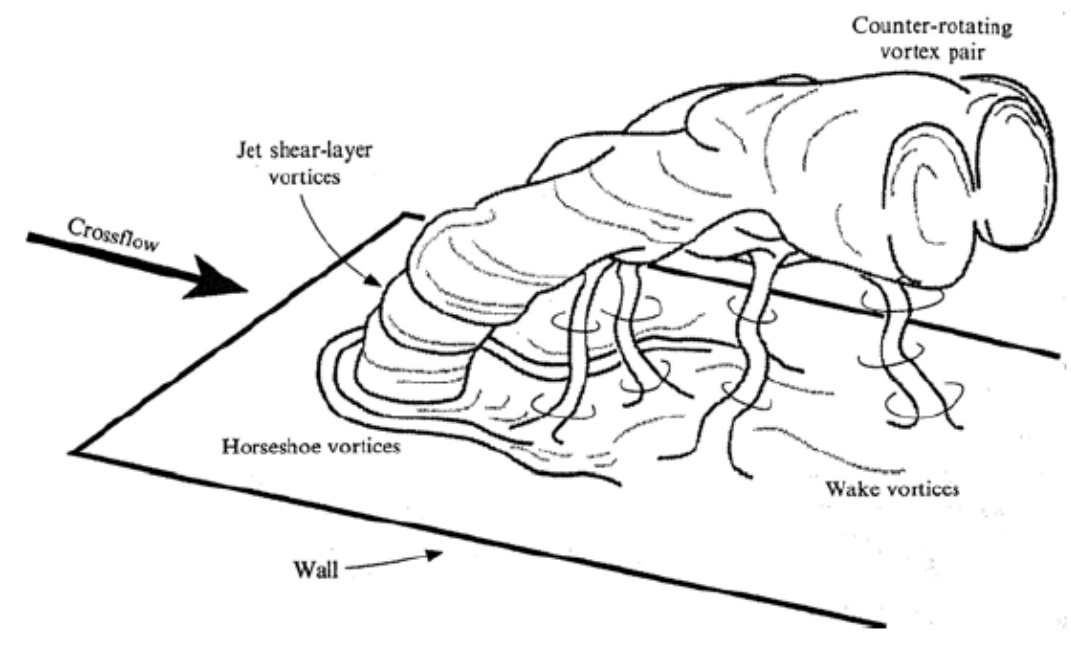

Figure 1: Anatomy of the transverse jet [1]

Additional flow features of the transverse jet, as illustrated in Fig. 1, include shear layer vortices, wake vortices, and the horseshoe vortex. Kelso, Lim, and Perry [5] studied various flow features of the JICF for a range of velocity ratios from 2-6 using hot-wire anemometry to examine the evolution of shear layer roll-up. Lim, New and Luo [6] then conducted a qualitative experiment on vortex formation by releasing a dye tracer at specific locations around the issuing jet. They found that the shear layer vortices that develop at the jet/crossflow interface coalesce as the jet propagates and eventually create the counterrotating vortex pair. Figure 2 shows the process by which this occurs for a typical transverse jet. On the upstream side of the jet, small scale vortical rings form and move fluid toward the downstream side. These vortices are wrapped up to the inside and form continually building larger-scale structures until the counter rotating vortex pair is formed. This is primarily how the jet entrains fluid from the crossflow at low velocity ratio and one of two methods by which the jet obtains crossflow momentum, the other being pressure drag. As the velocity ratio increases to approximately 10 and higher, the near-field of the jet behaves much like a free jet. With respect to wake vortices, Fric and Roshko [3] conducted a flow visualization study that concluded that the wake structures originate from the crossflow boundary layer. 
The pressure gradient downstream of the jet tends to suck up the slow-moving boundary layer fluid, creating vertical momentum and a tornado-like structure. Depending on the velocity ratio, the wake vortices will entrain a proportional amount of jet fluid. This was confirmed by Smith and Mungal [7] using planar laser-induced fluorescence.

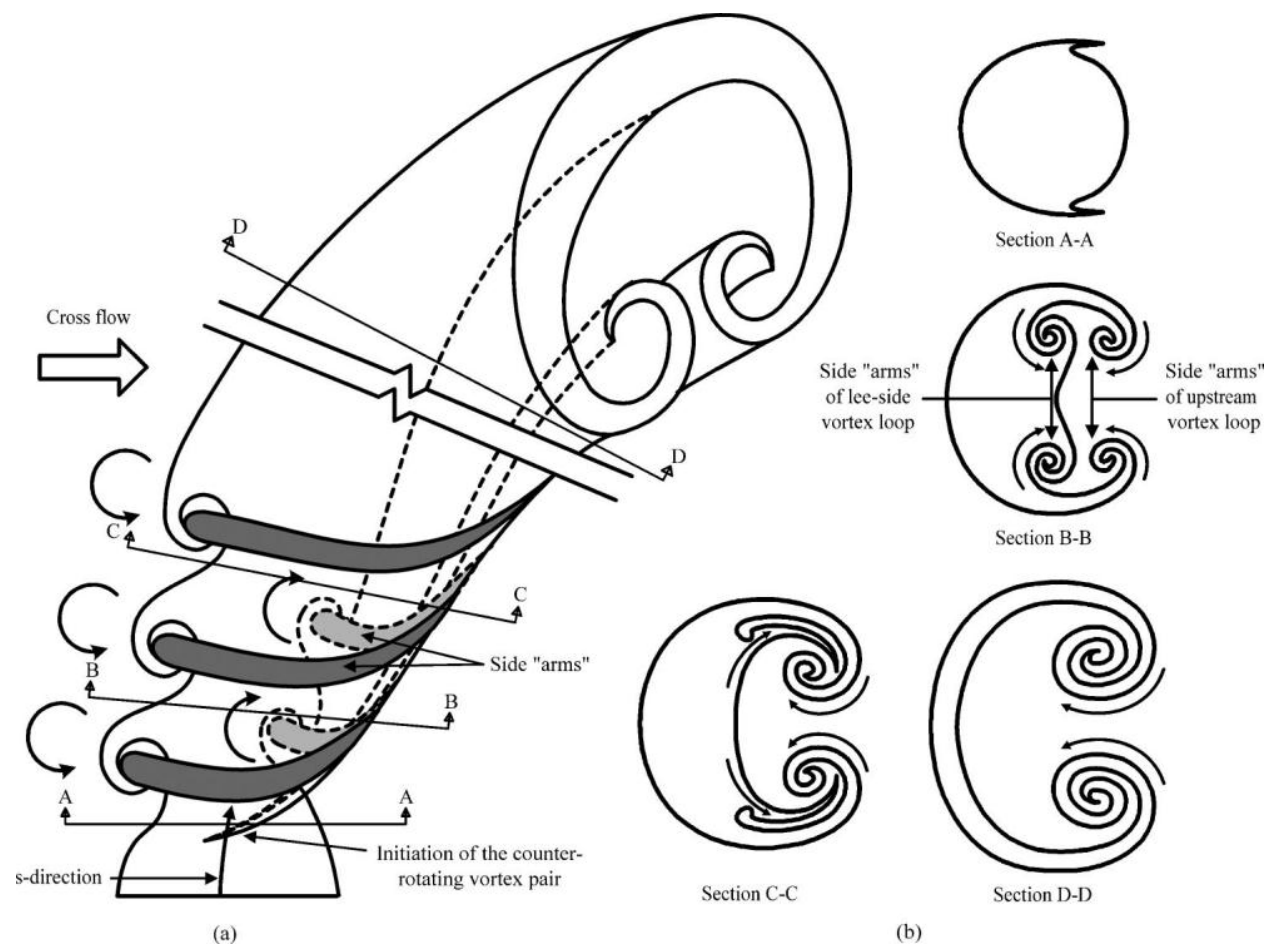

Figure 2: Evolution of shear layer wrap-up into CVP. Lim et al. [5]

The last significant secondary flow structure is the horseshoe vortex (also known as a "necklace" vortex by some authors). Kelso and Smits [8] studied the interaction between a transverse jet and the incoming boundary layer using hydrogen bubble wire visualization. Their conclusions were that the vortex can manifest in three regimes: steady, oscillating or coalescing. Since the vortex forms near the wall, it more closely approximates a cylinder in crossflow and the Strouhal numbers match accordingly. Overall, this feature does not tie strongly into bulk mixing and would only be of interest for heat transfer between a crossflow and the wall.

The JICF incorporates a variety of variables and is consequently difficult to generalize. Figure 3 lays out the basic setup for a transverse jet. Among these variables are jet and crossflow velocities $\left(\mathrm{v}_{\mathrm{j}}\right.$ and 
$\left.\mathrm{v}_{\mathrm{c}}\right)$, densities $\left(\rho_{\mathrm{j}}\right.$ and $\left.\rho_{\mathrm{c}}\right)$, viscosities $\left(\mu_{\mathrm{j}}\right.$ and $\left.\mu_{\mathrm{c}}\right)$, as well as jet diameter $(\mathrm{d})$. Additionally, boundary layer parameters such as displacement thickness $(\delta)$ in the crossflow and the jet velocity profile affect the generation of wake structures.

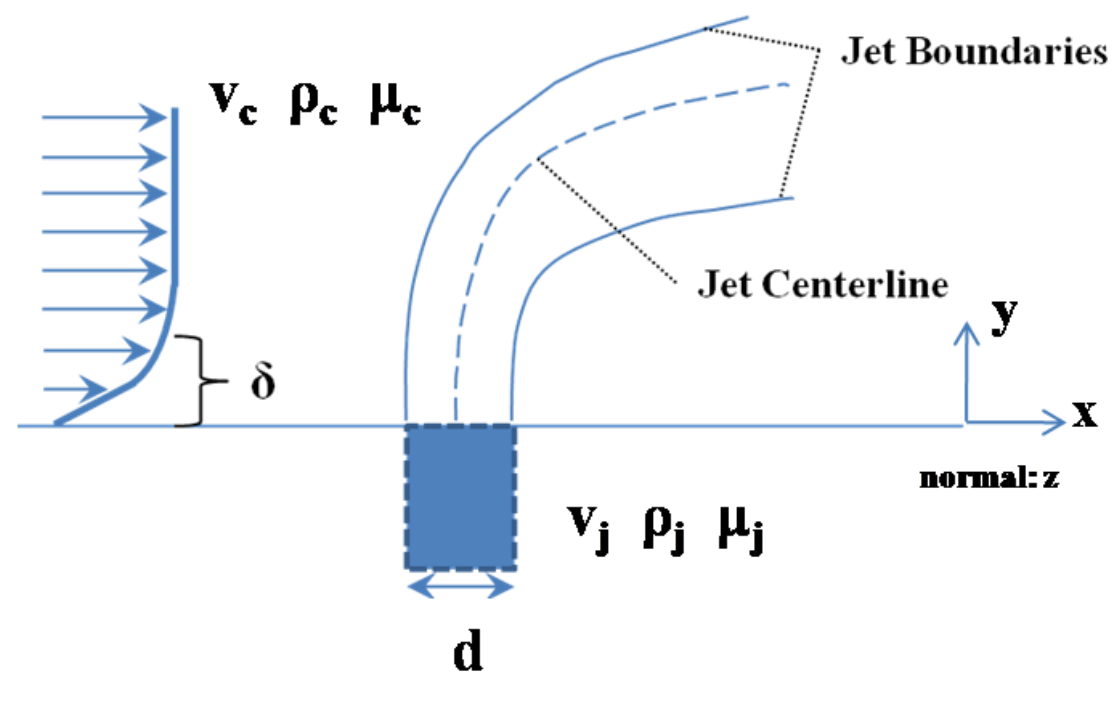

Figure 3: Variables affecting a generic JICF

When considering the specialized case of confined jets, other factors come into play, such as the number of jets and the relative spacing between them (related by the crossflow diameter, $D$ ). These variables can be formed into dimensionless ratios as summarized in Table 2. Scaling and trajectory laws are fairly well defined for a few classes of transverse jets. Studies include (but are not limited to) Keffer and Baines [9], Pratte and Baines [10], Wu [11], Kamotani and Greber [12], Maruyama et al. [13], Smith and Mungal [7], Hasselbrink and Mungal [4], Muppidi and Mahesh [14] and Mashayek et al. [15]. Among these are unconfined single jets and confined single jets. When considering interactions of multiple jets, Kamotani and Greber [16] found that scaling laws for single jets do not adequately describe the flowfield. Each of these three areas will now be discussed in more depth. 


\begin{tabular}{|ll|cl|}
\hline Density Ratio & $\frac{\rho_{j}}{\rho_{c}}$ & $\begin{array}{c}\text { Momentum } \\
\text { Flux Ratio }\end{array}$ & $J=\frac{\rho_{j} v_{j}^{2}}{\rho_{c} v_{c}^{2}}$ \\
\hline Diameter Ratio & $\frac{d}{D}$ & $\begin{array}{c}\text { Blowing } \\
\text { Ratio }\end{array}$ & $r=\sqrt{\frac{\rho_{j} v_{j}^{2}}{\rho_{c} v_{c}^{2}}}$ \\
\hline Velocity Ratio & $\frac{v_{j}}{v_{c}}$ & $\begin{array}{c}\text { Momentum } \\
\text { Ratio }\end{array}$ & $\beta=\frac{\rho_{j} v_{j}^{2} d^{2}}{\rho_{c} v_{c}^{2} D^{2}}$ \\
\hline
\end{tabular}

Table 2: Common dimensionless ratios from literature

\subsection{The Single JICF: Unconfined Case}

Extensive attention has been given to an unconfined single jet-in-crossflow, as this configuration is easiest to access with measurement techniques and has fewer variables. Two features of the jet are of general interest to the designer: trajectory scaling (synonymous with jet penetration) and scalar mixing. Early work was conducted by Keffer and Baines [8] and Pratte and Baines [9], who discovered that jets primarily follow a trajectory of the form

$$
\frac{y}{r d}=G_{1}\left(\frac{x}{r d}\right)^{G_{2}}
$$

where $x$ and $y$ are the horizontal and vertical coordinates from the center of jet injection, $r$ is the blowing ratio, $d$ is the jet diameter and $\mathrm{G}_{1}$ and $\mathrm{G}_{2}$ are empirical constants to fit experimental data. These studies concluded that in general, $\mathrm{G}_{1} \approx 1.6$ and $\mathrm{G}_{2} \approx 1 / 3$. However, as summarized by Magason [17] the spread of data is somewhat large $\left(1.2<\mathrm{G}_{1}<2.6\right.$ and $\left.0.28<\mathrm{G}_{2}<0.34\right)$. Hasselbrink and Mungal [11] suggested that some of this scatter may be due to how the jet velocity is defined. An integral conserved quantity gives the best estimation of jet behavior and is only identical to the mean velocity for a uniform (top-hat) velocity profile at the jet exit. Broadwell and Breidenthal [18] produced an analytical argument for the rd scaling law using self-similarity as an argument. A more robust scaling law for the jet centerline penetration was necessary. Kamotani and Greber [12] addressed this by developing the form 


$$
\frac{y}{r d}=G_{1} r^{G_{3}}\left(\frac{x}{r d}\right)^{G_{2}}
$$

where $G_{3}=0.30$. Smith and Mungal [6] were able to corroborate this relationship in the near field of the jet for blowing ratios between 10 and 25. By scaling the data with the blowing ratio, Kamotani and Greber were able to account for different regimes associated with the blowing ratio (also equal to the square root of the momentum flux ratio). This has prompted most subsequent studies to focus on blowing ratio or momentum flux ratio as the parameter of interest for a unified jet scaling law.

Muppidi and Mahesh [14] presented a method for perhaps better collapsing trajectory data by using integral methods accounting for features such as the crossflow boundary layer and the velocity distribution of the jet. They were perhaps the first to use direct numerical simulation (DNS) for this task, as Reynolds numbers associated with typical transverse jets are somewhat higher than is computationally feasible for DNS simulations. Their model infers pressure drag as the dominant source of jet deflection in the near field, rather than entrainment of crossflow fluid momentum. New, Lim and Luo [19] evaluated the DNS results with historical data and found it actually correlates worse than the form originally posed by Keffer and Baines [9]. For a top-hat jet profile, $\mathrm{G}_{1}=1.65$ and $\mathrm{G}_{2}=0.25$. For a parabolic profile, $\mathrm{G}_{1}=$ 1.96 and $\mathrm{G}_{2}=0.31$. The parabolic profile is similar to turbulent pipe flow and falls close to the value of $1 / 3$ estimated by various sources.

Overall, there appears to be no universal scaling law defined for a broad range of transverse jets. Given the wealth of parameters affecting jet trajectories (both velocity and scalar), this is not entirely surprising. For the current effort, the best path forward is to utilize data more similar to the current configuration. These include a confined single jet-in-crossflow and multiple confined jets-in-crossflow. 


\subsection{The Single JICF: Confined Case}

In the chemical processing industry, single confined transverse jets hold a particular interest for mixing in order to control the rate at which a chemical reaction occurs. The pipeline or "tee" mixer is commonly used to mix two constituents at specified rates to control a chemical reaction. With specified flow rates, it is then of interest to create a geometry that promotes effective mixing in both the near and far field. Maruyama et al. [13] investigated the optimum conditions for a single jet and dual jets while varying the tangential offset to introduce swirl. They used the second moment $M$ about the mean temperature to quantify the scalar non-uniformity, defined as

$$
M=\frac{1}{A} \int_{A} \frac{(T-\bar{T})^{2}}{\bar{T}^{2}} d A
$$

where $A$ and $d A$ are the cross-sectional and infinitesimal areas of interest, $T$ is the time-averaged mean local temperature and $\bar{T}$ is the spatial mean of the entire time-averaged temperature field. This value is often normalized by the value of $M$ at the injection location, given by

$$
M_{0}=\frac{\left(\frac{Q_{c}}{Q_{j}}\right)^{2}\left(\frac{d}{D}\right)^{2}+1}{\left(\frac{d}{D}\right)^{2}+1}
$$

where $Q_{c}$ and $Q_{j}$ are the crossflow and jet volumetric flow rates. In general, they observed a "bucketshaped" curve at downstream measurement locations, indicating an optimum velocity ratio for a given diameter ratio. Figure 4 illustrates this trend for a diameter ratio $d / D=0.071$. 


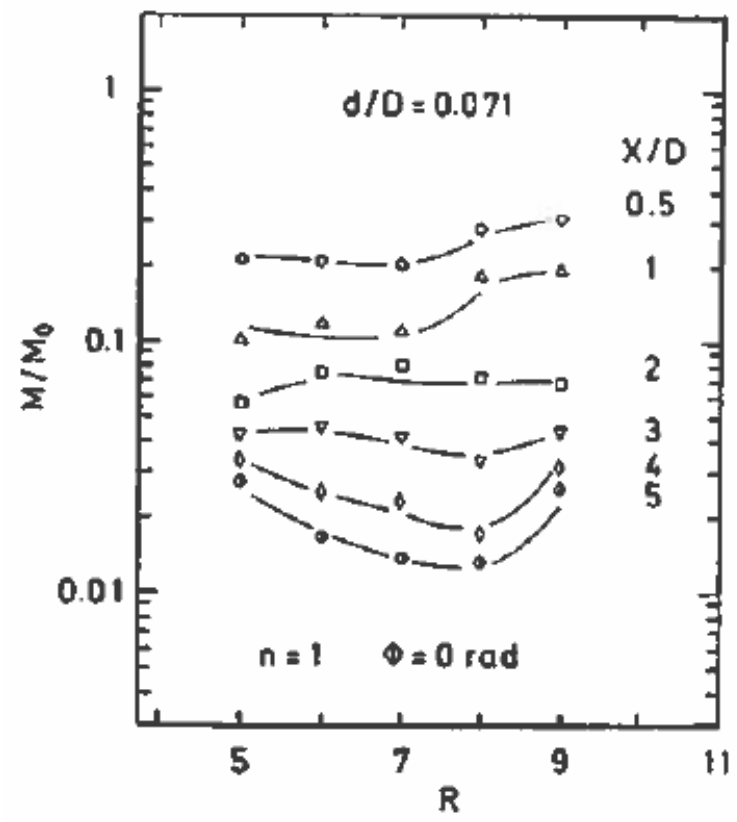

Figure 4: Second moment for single jet injection $(d / D=0.071)$. Maruyama et al. [13]

For this case, the optimum velocity ratio is approximately 8 in the flow field. Similar trends were seen with other configurations. Maruyama and colleagues developed relationships for the optimum velocity ratio as a function of the diameter ratio for the single and dual jet configurations. The current data set will be compared with these results.

Sroka and Forney [20] expanded upon the results of Maruyama et al. and others and developed a theory to explain different regimes of single jet mixing. They found that the second moment described by equation 4 is a function of both the momentum ratio, $\beta$, and the downstream pipe location, $x / D$, such that

$$
M=k_{1} \beta\left(\frac{x}{D}\right)^{-4 / 3}
$$

where $k_{1}$ is a constant equal to 0.25 . They correlated data from multiple sources and found three distinct mixing behaviors outlined in figure 5 . In their nomenclature, the term $\left(l_{m} / D\right)^{-2}$ is equivalent to the momentum ratio $\beta$ for a density ratio of unity. The vertical axis represents equation 6 multiplied by the inverse of the $x / D$ term for each data set, the collapse of the data over various values of $x / D$. The jet 
behaves like a wall source at low momentum ratios (up to $\beta=0.005$ ), trends to a centered transverse jet at moderate momentum ratios (up to $\beta=1$ ) and finally a jet impacting the opposing wall, breaking up the coherent structures of the jet and increasing interfacial area between the jet and crossflow. In general, they find that in order to achieve better mixing, simply raising the momentum ratio as high as possible will accomplish this.

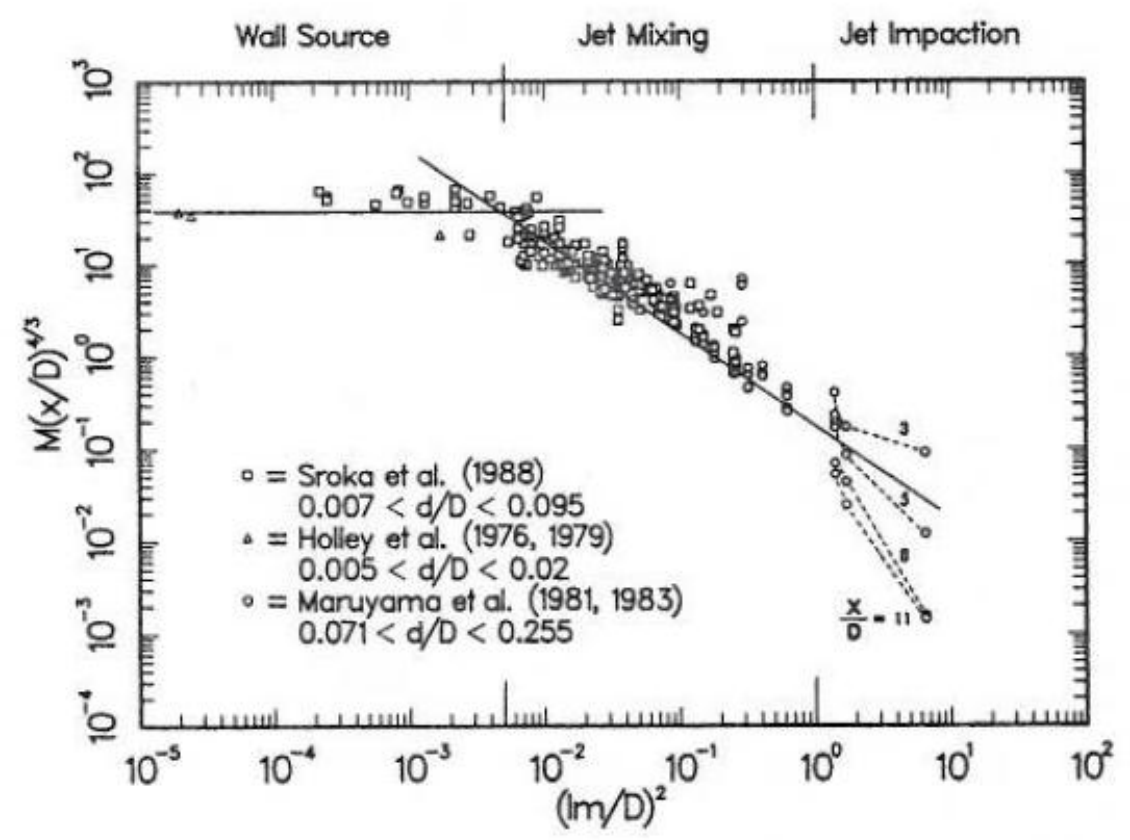

Figure 5: Correlation of second moment data for $(x / D) \leq 12$. Sroka and Forney [20].

While the scaling law developed by Sroka and Forney does appear to work well for single jets, it does not explain interactions between multiple jets. The current study uses the second moment correlation utilized by [13] and [20] for direct comparison of mixing performance.

Pan and Meng [21] investigated mixing in a pipeline tee using PIV and PLIF, providing a much clearer view into the scalar distribution in a transverse jet. They concluded that under moderate velocity ratios, the mixing behavior is similar to an unconfined crossflow. The jet centerline concentration decayed as $x^{-2 / 3}$, which correlates with the data collected by Sroka and Forney [20]. 


\subsection{Multiple Confined JICF}

In a typical gas turbine combustor, dilution air is introduced slightly downstream of the primary combustion to achieve more uniform gases and lower gas temperatures entering the turbine. This extends turbine life by minimizing the amount of temperature variations seen by the turbine. The mixing properties of multiple jets in crossflow were parameterized by Holdeman, Walker and Kors [22]. This study explored multiple air jets issuing into subsonic crossflow with momentum flux ratios between 6 and 60. They concluded that for a given momentum flux ratio, their existed an optimum spacing between jets, below and above which mixing was worse. Their parameter for evaluating mixedness of the bulk flow was dimensionless temperature,

$$
\theta=\frac{T_{c}-T_{i}}{T_{c}-T_{j}}
$$

where $T_{c}$ represents the crossflow temperature, $T_{i}$ represents the local temperature, and $T_{j}$ represents the jet temperature. It is also noteworthy that each of these temperatures is time-averaged. Figure 6 details Holdeman's general arrangement. Kamotani and Greber [12] expanded on this work in their 1974 NASA report. They arranged jets downstream of other jets and varied the spacing $(2<\mathrm{S} / \mathrm{D}<6)$ and momentum ratio $(8<\mathrm{J}<72)$. They create quantitative maps of dimensionless temperature in much the same way as Holdeman et al. [22] but make no recommendation for an optimum configuration. They suggest that qualitatively, the temperature decay mimics a single jet in the upstream region and a two dimensional (slot) jet in the farfield region. A more quantitative evaluation of mixing performance was necessary. 


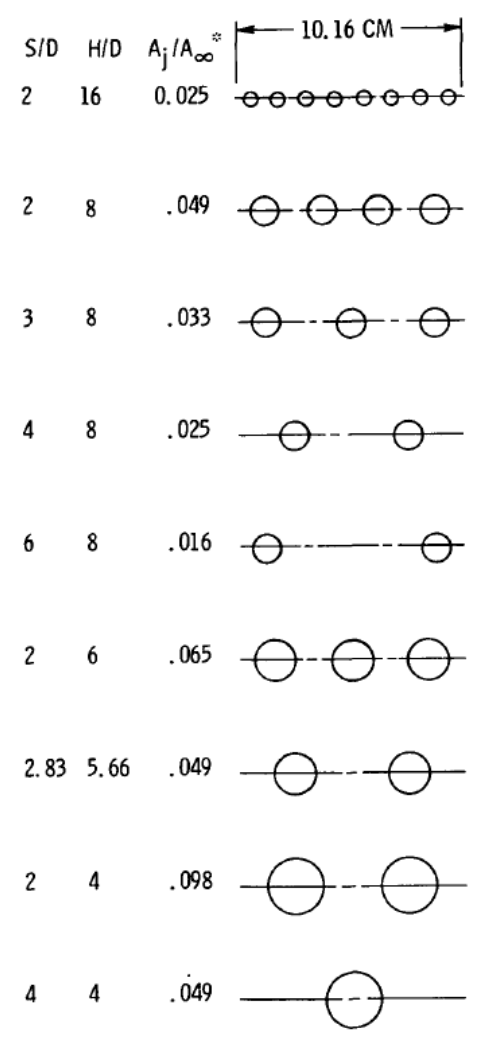

Figure 6: Two-dimensional duct configuration for evaluating multiple JICF mixing. Holdeman et al. [23]

In 1993, Holdeman [23] released an extensive review of NASA work in this area. Holdeman investigated experimental and computational results of single, double, and opposed rows of jets in a twodimensional duct, again using dimensionless temperature (Eq. 3) as a figure of merit for flow mixedness. The most significant conclusions were as follows:

- momentum flux ratio was the most significant discriminator of mixing performance for otherwise similar jets,

- temperature distributions were qualitatively similar when the jet spacing and the blowing ratio (square root of momentum flux) were inversely proportional, independent of jet diameter.

The first conclusion had been surmised by Kamotani and Greber [12] when they developed Eq. 2, such that blowing ratio affected jet penetration into the crossflow and Holdeman's data agrees with this. However, Holdeman's second conclusion is somewhat revolutionary. The body of evidence indicated that 
the size and shape of the jets have only second-order influence on mixing, as long as the criterion for jet spacing versus blowing ratio was held inversely proportional. To a designer, this allows greater flexibility when designing a mixing chamber, such that any size jet diameter may suffice. The designer may then choose the momentum flux ratio that optimizes mixing and design the orifice spacing and diameter such that flow rate requirements are met. However, it should be noted that while this relationship appears valid over the range of conditions considered, empirical correlations should be used with caution when moving outside of Holdeman's design space (to include jet number, momentum flux and diameter ratios).

Holdeman and coworkers conducted an extensive study (see Fig. 6 for geometry) with widely ranging parameters of density ratio, momentum flux ratio, orifice size and spacing. Table 3 shows a summary of these variables.

\begin{tabular}{|lr|}
\hline Independent variables: & Range \\
\hline Downstream distance, $\mathrm{x} / \mathrm{D}$ & 0 to 2 \\
Density Ratio & 0.5 to 2.2 \\
Momentum Flux Ratio & 5 to 105 \\
Orifice diameter & 4 to 16 \\
Orifice traverse (circumferential) spacing & 2 to 6 \\
Orifice aspect ratio & 0.36 to 2.8 \\
Area ratio (exit/inlet) & 0.33 to 1 \\
Radius of curvature in x-r plane, $\mathrm{R}_{\mathrm{c}} / \mathrm{H}_{0}$ & 0.25 to $\infty$ \\
Radius of curvature in r-z plane, $\mathrm{R}_{\mathrm{t}} / \mathrm{H}_{0}$ & 0 to $\infty$ \\
Variable mainstream, $\theta$ & 0 to 0.5 \\
& \\
\hline Derived variables: & \\
\hline $\mathrm{C}_{0}=\left(\mathrm{S} / \mathrm{H}_{0}\right) \sqrt{J}$ & 0.5 to 10 \\
\hline
\end{tabular}

Table 3: Transverse jet design space. Holdeman, J.D. [23]

From his study, Holdeman concluded that the design of an optimal can-annular mixer could be governed by a few relationships. He collapsed his experimental data for a two-dimensional duct to form empirical relationships and used it to estimate performance for an axisymmetric can. The circumferential spacing between jets for the round duct may be defined using

$$
S=\frac{2 \pi R_{1 / 2}}{n}
$$


where $\mathrm{R}_{1 / 2}$ is

$$
R_{1 / 2}=\frac{H_{0}}{\sqrt{2}}
$$

Physically, $\mathrm{R}_{1 / 2}$ represents the radius inside a can which divides it into two equal areas.

Penetrating jets to this distance would then theoretically allow neighboring jets to intersect one another and promote uniformity. Holdeman's finding that the orifice spacing ratio $\left(\mathrm{S} / \mathrm{H}_{0}\right)$ is inversely proportional to the square root of momentum flux can be given by

$$
C_{0}=\left(S / H_{0}\right) \sqrt{J}
$$

where $\mathrm{C}_{0}$ is a correlation coefficient. The value of $\mathrm{C}_{0}$ depends on test conditions, but in general the data indicate that $\mathrm{C}_{0} \approx 2.5$ seems to be associated with good mixing. We may then combine Equations 4,5 and 6 to obtain an expression for the number of jets as a function of the optimum momentum flux ratio such that

$$
n=\pi \sqrt{2 J} / C_{0}
$$

For duct configurations with injection from only one side, $\mathrm{C}_{0}$ ranged between one and five. For two-sided injection, $\mathrm{C}_{0}$ ranged between 0.5 and 2.5. It should be noted that the data considered here finds that impingement actually impedes mixing when evaluated at $x / D$ values of $0.5-1$. Sroka and Forney [20] demonstrated for single confined jets that in order to produce effective mixing, impingement is almost a requirement when measured at $x / D$ values of 3-5.

Vranos et al. [24] conducted a study of a nominal can geometry to evaluate the effect of momentum flux ratio on mixture uniformity for a given axial cross section. They were among the first to use optical measurements for this purpose, incorporating an argon-ion laser and camera to analogize light intensity from Mie scattering with particle count. The momentum flux ratio varied from approximately 5 to 100 and density ratio between 1 and 2.77 . Their results are possibly the first to plot global mixing 
performance versus momentum flux ratio. Most of their results utilize the distribution of the mean mass fraction as the objective function for mixing, defined as

$$
\frac{C_{r m s}}{C_{\text {avg }}}=\frac{\sqrt{\frac{1}{N} \sum_{i=1}^{N}\left(C_{i}-C_{\text {avg }}\right)^{2}}}{C_{\text {avg }}}
$$

Where $C_{\text {avg }}$ is equal to

$$
C_{\text {avg }}=\frac{1}{N} \sum_{i=1}^{N} C_{i}
$$

Their parameter $C_{r m s} / C_{a v g}$ is essentially equal to the standard deviation divided by the mean. This is a useful parameter to investigate, however it can be shown that the standard deviation divided by the mean tends to drift as the jet to crossflow mass flow ratio changes. Vranos et. al. also define a relative unmixedness, $\mathrm{U}$ as

$$
U=\left(\frac{C_{r m s}}{C_{\text {avg }}}\right)\left(\sqrt{\frac{\bar{C}}{1-\bar{C}}}\right)
$$

where $\bar{C}$ is the fully mixed mass fraction of total flow that is jet fluid, equal to the ratio of the jet-to-total mass flow rate. It can be shown that using this ratio normalizes the flowfield to be constrained to mixing values between 0 (perfectly mixed) and 1 (perfectly segregated) regardless of the mean concentration value. This parameter will be called unmixedness, $\mathrm{U}$, throughout the remainder of this thesis.

Figures 7 and 8 illustrate this for two density ratios at axial locations of $x / D=0.6$ and 1.0. The jet shape is a rectangle with semi-circular ends. The number of slots is six and the equivalent diameter ratio is approximately 0.21 . The data indicates that there exists a local optimum momentum flux ratio near 30. This seems to diminish with downstream location for both cases of density ratio. The current study seeks to replicate this trend for the density ratio of unity. The results will vary slightly as the above data is for a slotted injector and the current study focuses on round jets. Additionally, the current effort places more emphasis on mixing further downstream $(x / D=3)$. 


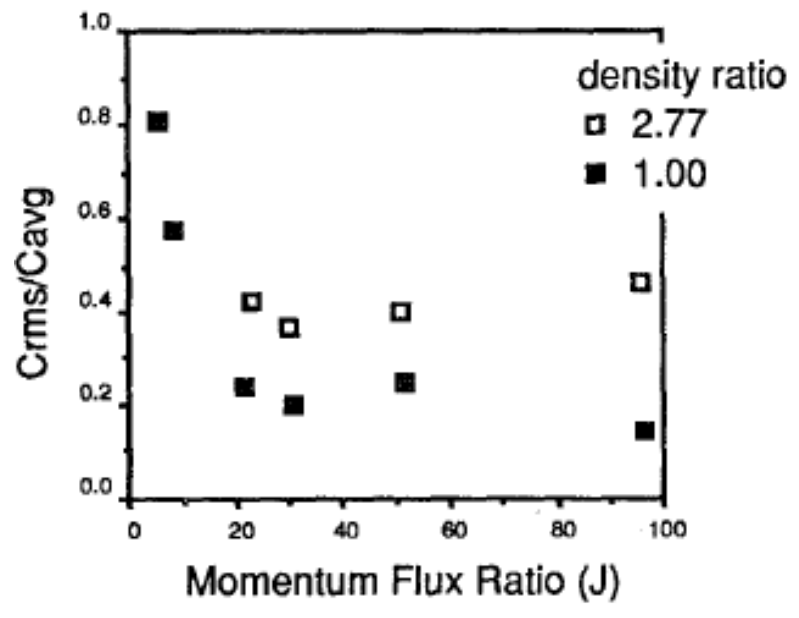

Figure 7: Six-jet mixing data for $\mathrm{x} / \mathrm{D}=\mathbf{0 . 6}$. Vranos et al. [24]

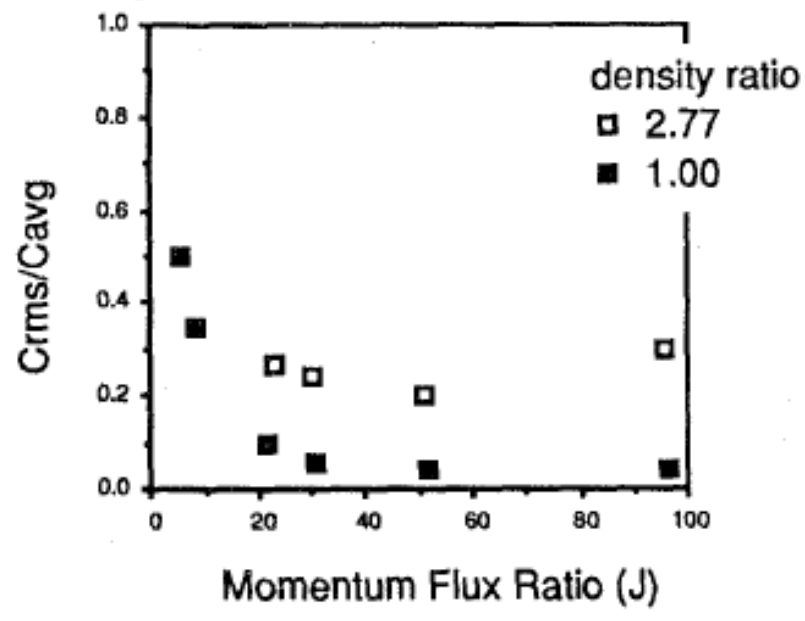

Figure 8: Six-jet mixing data for $\mathrm{x} / \mathrm{D}=\mathbf{1 . 0}$. Vranos et al. [24]

Additionally, Vranos et al. [24] documented the effect of the number of jets against mixing performance at the same two axial locations $(x / D=0.6$ and 1.0) for various momentum flux ratios. Figures 9 and 10 illustrate these trends. From these data, it is notable that six jets offered the most promising performance over the range of momentum flux ratios of interest to the current study $(10<\mathrm{J}<$ 50). The effect is more prominent at the further downstream location $(x / D=1.0)$. Since the effect of the number of jet is easier to visualize when isolated for a single momentum flux ratio, figure 11 displays these data for a momentum flux of 50. In the near field $(x / D=0.6)$ the unmixedness decreases monotonically with number of jets. Moving to $x / D=1.0$ indicates some decrease in mixing effectiveness for 12 jets, suggesting that a lower number may be optimal for far-field mixing. 


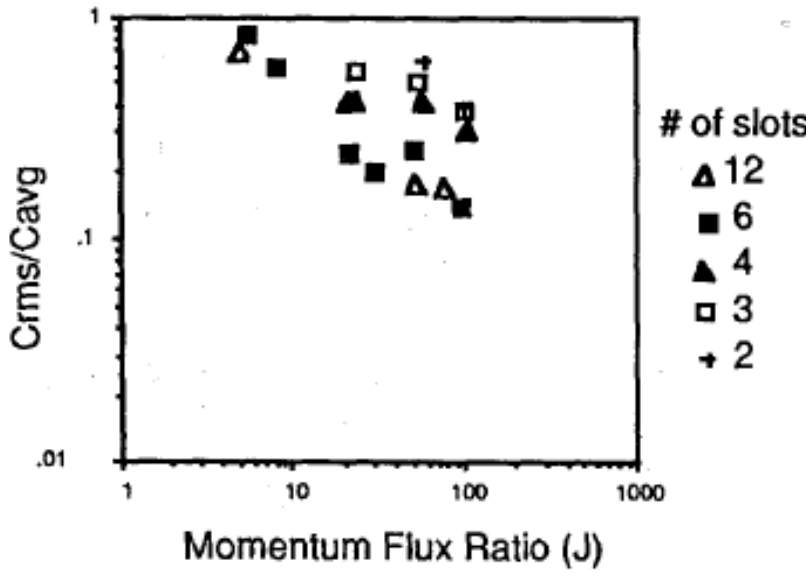

Figure 9: Mixing as a function of number of jets and momentum flux ratio at $\mathrm{x} / \mathrm{D}=\mathbf{0 . 6}$. Vranos et al. [24]

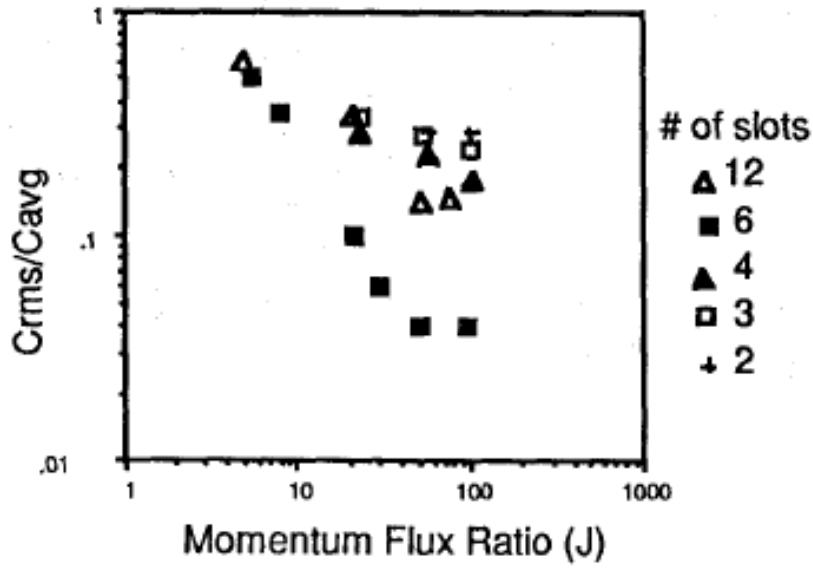

Figure 10: Mixing as a function of number of jets and momentum flux ratio at $\mathrm{x} / \mathrm{D}=\mathbf{1 . 0}$. Vranos et al. [24]

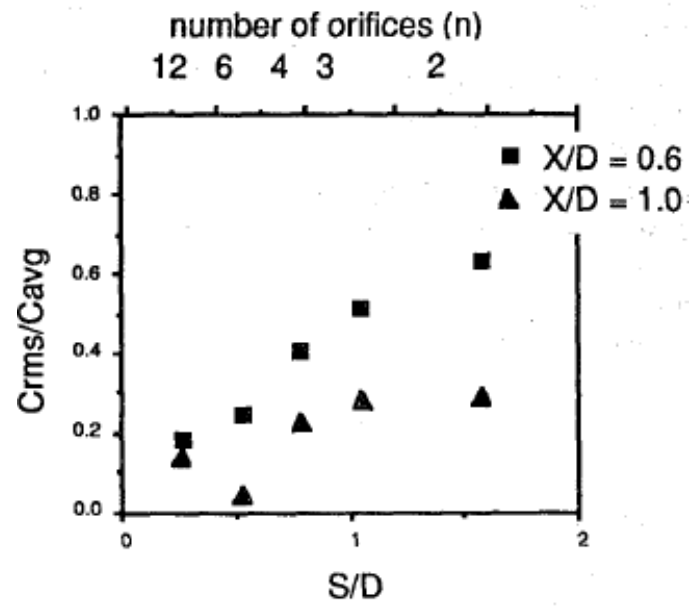

Figure 11: Effect of number of jets on mixing $(\mathrm{J}=\mathbf{5 0})$. Vranos et al. [24]

Hatch et al. [25] investigated axisymmetric mixing of cold air jets into a heated crossflow. They studied various configurations of eight circumferentially-spaced jets at three different momentum flux ratios $(25,52$ and 80$)$. They used both circular jets and slots of equivalent area, at aspect ratios of 4 and 8 . Their data was collected by mapping a one-quarter pipe area using a temperature probe and assuming symmetry. They used non-dimensional temperature (Eq. 6) as their flow variable and calculated a mixture uniformity given by

$$
U=\sqrt{\frac{1}{A} \sum_{i=1}^{N} a_{i}\left(\theta_{i}-\theta_{e q}\right)^{2}}
$$


where $\theta_{e q}$ indicates the ideal dimensionless temperature based on the mass flow ratio and the temperatures of the hot and cold streams. Their findings indicate that there is an optimum penetration for a given geometric configuration. Underpenetration will result in a relatively unmixed core of crossflow fluid and overpenetration will conversely result in jets coalescing into a high jet-fluid core. Additionally, penetration depends on the coupling between orifice geometry and the momentum flux ratio. Increasing the aspect ratio of the slanted slots reduces overall penetration and enhanced mixing only in the near-wall region. Increasing the angle of the slots with respect to the mainstream also reduced penetration and enhances circumferential mixing.

Holdeman et al. [26] summarizes a number of these efforts and uses the data to support the aforementioned scaling law (Eq. 9). Figure 12 shows a general trend noted by Holdeman for the case of eight jets. Non-uniformity tends to drop to a local optimum momentum flux ratio, rises for some finite regime, and then continues to decrease. The degree to which mixing improves and the value of momentum flux ratio at which this local optimum occurs seems to have some dependency on the shape of the jet and (by extension) the jet diameter. A significant fact presented by the data in Fig. 12 is that Holdeman's $\mathrm{C}_{0}$ value is approximately 2.5 for the case of $\mathrm{J}=20$ and eight jets. This provides additional credence to Holdeman's scaling law. The current study investigates this trend to determine if the local optimum exists for lower jet numbers and for the studied configurations and identify if there is any sensitivity to jet number or diameter ratio. 


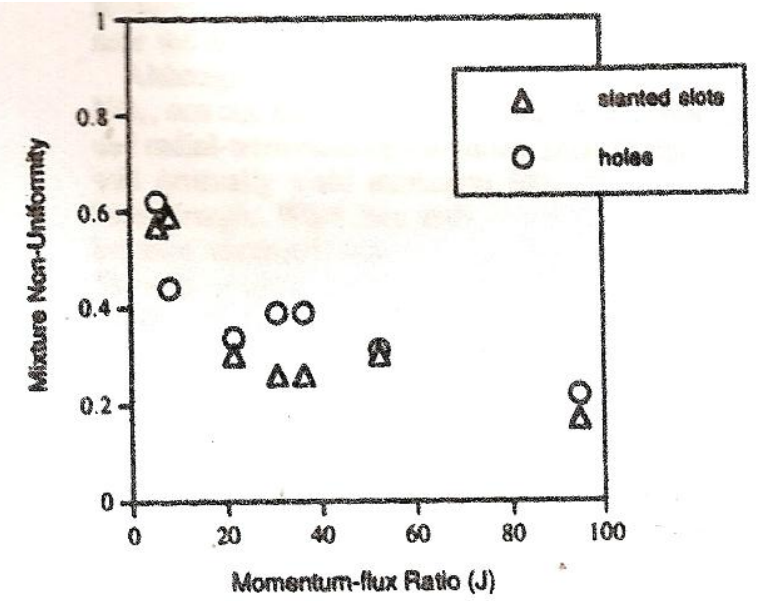

Figure 12: Mixing non-uniformity for $n=8$ configurations at $x / D=1.2$. Holdeman et al. [24]

\section{$2.5 \quad$ Measurement Techniques}

Planar laser-induced fluorescence (PLIF) is a technique that uses the fluorescent properties of a tracer to visualize a flow-field in a non-intrusive manner. A fluorescent tracer is introduced upstream into one or several fluid streams to make it distinctive from the primary flow. An appropriate laser is selected based on the absorption spectrum of the fluorescent tracer to maximize absorbed energy. This laser is placed perpendicular to the flow stream and shaped using spherical and cylindrical lenses. The resulting light sheet then enters the test section through a clear window (silica, quartz, or acrylic) and produces a visible cross section, either lateral-axial (viewing perpendicular to flow direction) or bi-lateral (parallel to flow direction). Figures 13 and 14 denote these respective setups. A camera or other photo-sensitive device is placed perpendicular to the visible cross section and records the flow at a rate appropriate for analysis. 


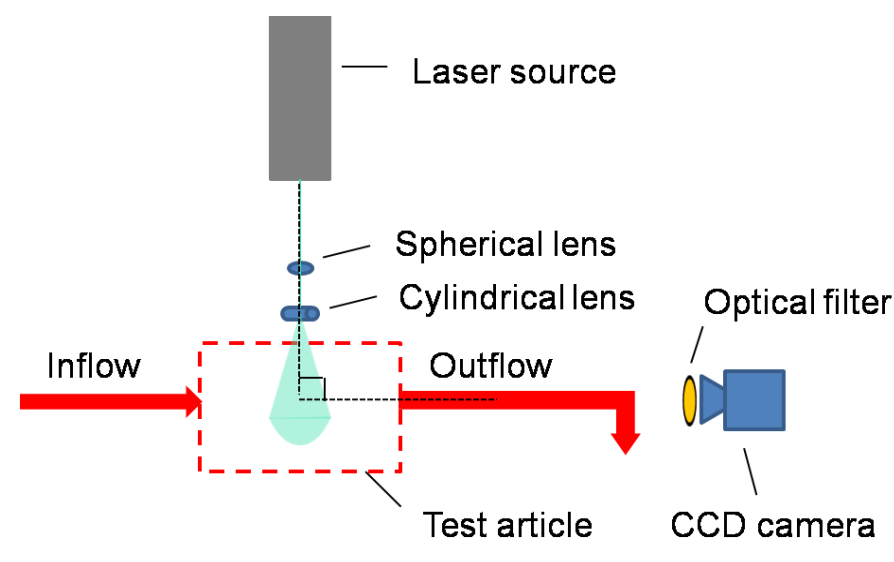

Figure 13: Bi-lateral PLIF setup

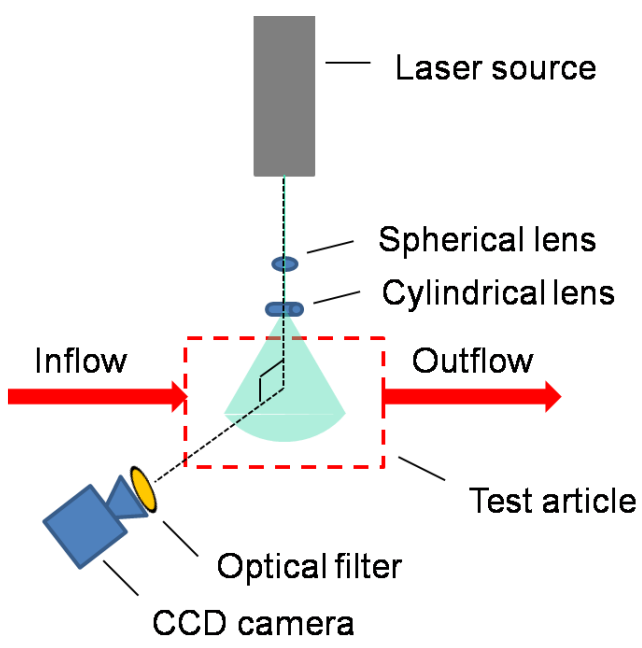

Figure 14: Lateral-axial PLIF setup

Laser-induced fluorescence (LIF), which is similar to the above except one-dimensional, has been employed as a measurement technique since the mid 1980s. It is first documented by Koochesfahani and Dimotakis [27] to study the mixing behavior in shear layers. The configuration they used involved a $3 \mathrm{~W}$ argon-ion laser as the light source, sodium fluorescein as the tracer, a custom-built analog-to-digital converter and linear photodiode array capable of a $0.8 \mathrm{~ms}$ response time at a frame rate of 1250 frames per second. This high-temporal resolution data array allowed for linear data at incremental time steps to be "stitched" together to form a composite image of shear-layer vortical structures as they passed by an axial location in the test section. Figure 15 illustrates their setup. 


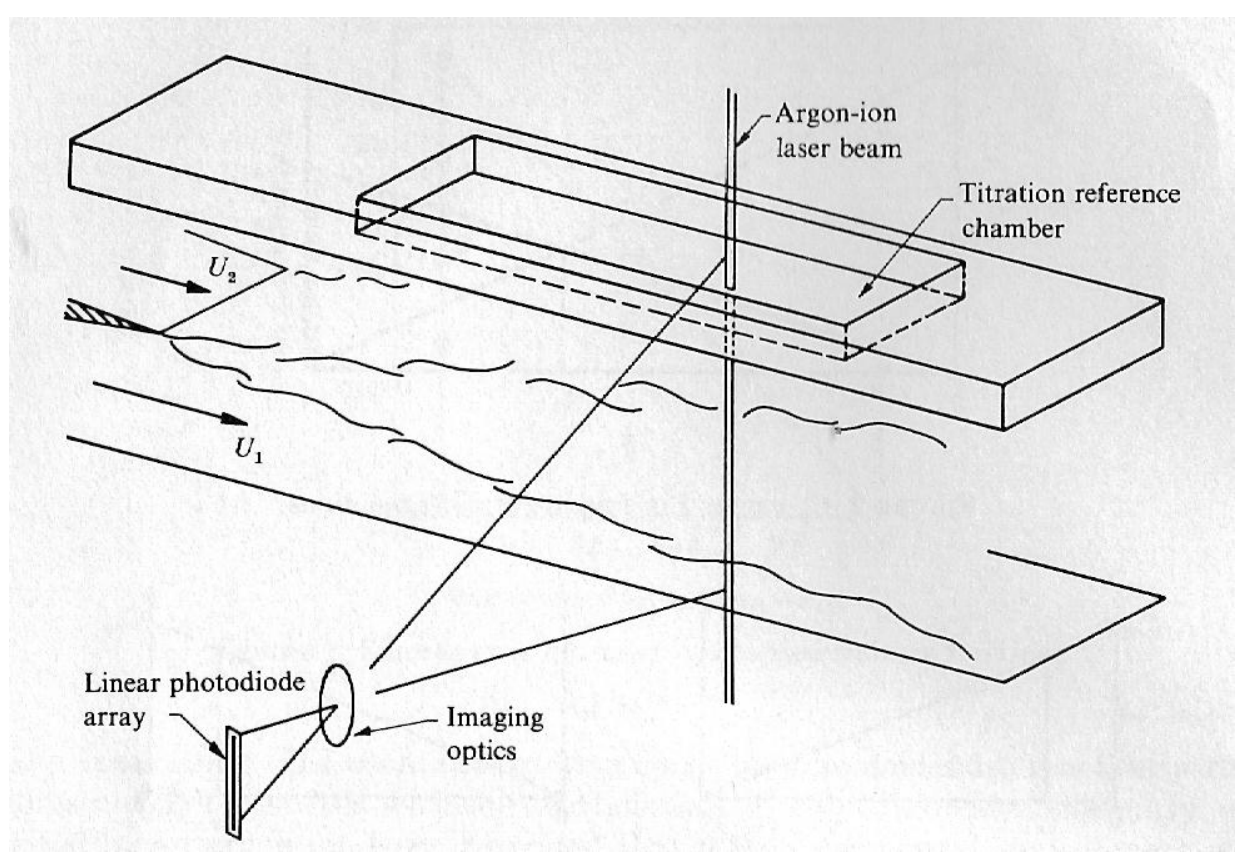

Figure 15: LIF optical setup for shear layer experiment. Koochesfahani and Dimotakis [25]

In a follow-up LIF study, Koochesfahani and Dimotakis [28] studied the effects of shear layer mixing under high Schmidt number conditions using an acid-base reaction. This differed from the previous study in that sodium fluorescein fluorescence is suppressed under acidic conditions. Water was used for both shear layer streams, however each had their $\mathrm{pH}$ adjusted such that despite molecular diffusion between the streams, fluorescence would be limited to areas containing only fluid originating from the tracer-marked flow. The same hardware and optical setup was used and they were able to conclude that below the saturation level for their photodiode array, output voltage was linearly proportional to fluorescence intensity and consequently, dye concentration.

In 1987, Walker presented a summary overview of the LIF technique [29] and various considerations that must be made regarding experimental setup. He provides a comprehensive background of the theory behind fluorescence and the considerations that must be made when quantifying fluorescence. His fluorescent dye of choice was sodium fluorescein. Among the major conclusions he lists are:

- fluorescence output intensity should ideally be linear with laser power intensity; 
- laser attenuation should be negligible until a so called "saturation" concentration, at which point the number of tracer molecules begin to appreciably attenuate the laser signal from reaching neighboring tracer molecules;

- fluorescence output intensity should be fairly linear with concentration until a saturation limit, where output signal begins to roll-off (Fig. 16);

- the $\mathrm{pH}$ of the bulk fluid and the injection fluid play an important role in promoting (basic) or suppressing (acidic) fluorescence output (Fig. 17);

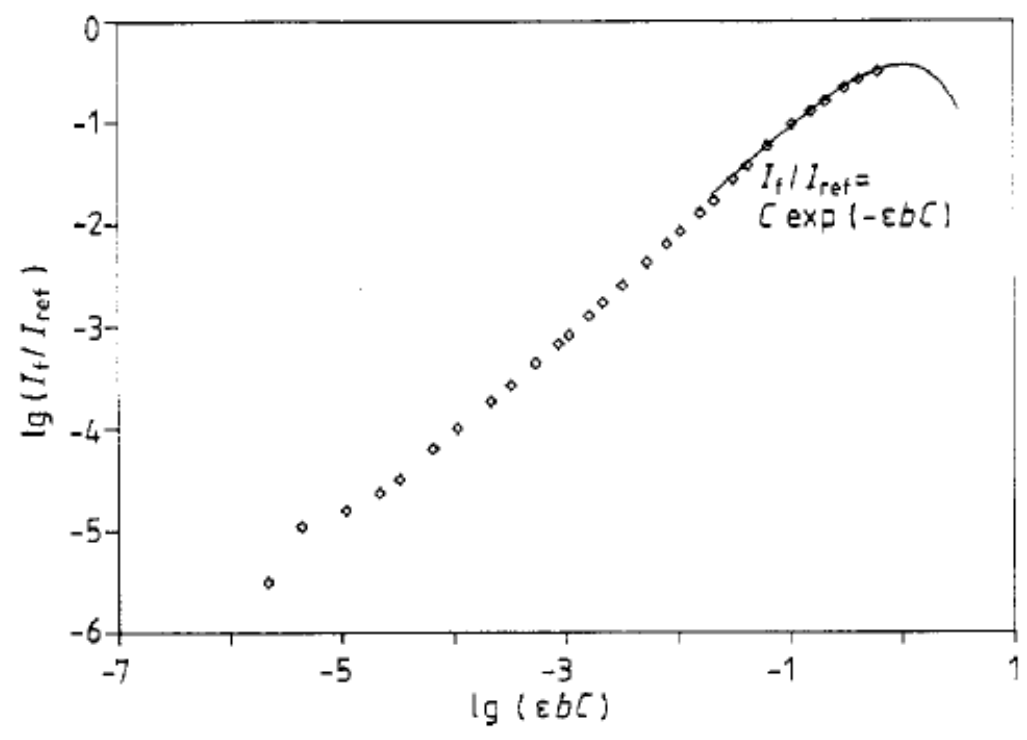

Figure 16: Fluorescence intensity as a function of concentration. Walker [29] 


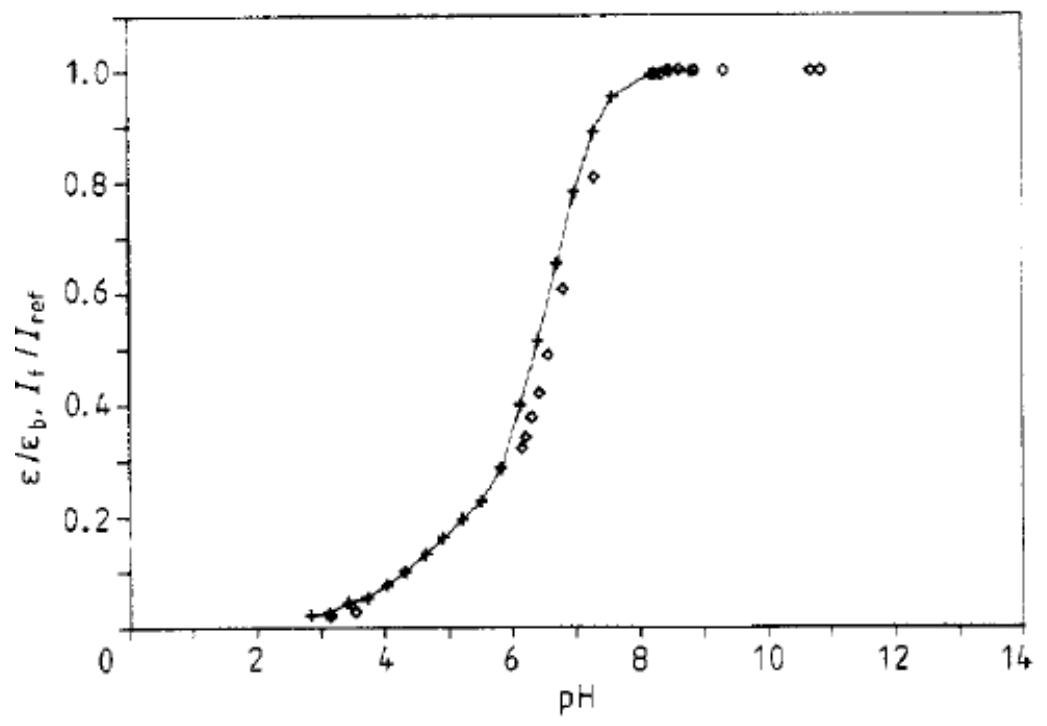

Figure 17: Fluorescence intensity as a function of fluid pH. Walker [29]

Extension of the LIF concept was made to PLIF over a variety of experiments through the last 25 years. Koochesfahani, Dimotakis and Broadwell [30] were able to study shear layer mixing using the PLIF technique with a $3 \mathrm{~W}$ argon-ion laser. They used the fluorescence suppression of acid in an acid-base reaction across the mixing layer in order to separate the two streams from data collection. As the acid side mixed with the base, the strength of the fluorescence rose to some finite expected value based on the mass flow ratio of the two streams. The data was collected using 35-mm film and analyzed qualitatively. Koochesfahani and MacKinnon [31] built upon the success of this data collection setup by using a CCD camera to extract quantitative data of shear layer mixing. Their field of view allows for a pixel resolution of approximately $200 \times 200 \mu \mathrm{m}$, four times larger than their expected scalar diffusion scale (Batchelor scale). Consequently, their probability density functions of concentration are expected to be only an upper bound of molecular mixing. However, they note that since the camera resolution was not changed over the course of the experiment, they assert that differences in the probability density functions are expected to be reliable for comparison. Since liquid mixing experiments typically use water $(\mathrm{Sc} \approx 800)$ this causes the Batchelor scale to be very small, on the order or smaller than the Kolmogorov scale. Their conclusion is then very important for any experiment investigating mixing using liquids. Although the Batchelor 
scale may be too small to resolve for macro-level flow measurements, the probability density functions will be correct for first-order statistics (mean concentration) but may possess slight error for higher order statistics (variance, skewness, etc.).

Other fluorescent tracers besides sodium fluorescein have also been studied in different optical configurations. Van Cruyningen, Lozano and Hanson [32] investigated PLIF in a gaseous flow using biacetyl (2-3 Butanedione) and a pulsed XeF excimer laser $(\lambda=351 \mathrm{~nm})$. They studied a free jet issuing into a quiescent reservoir, making various corrections and calibrations for camera behavior, background lighting, and laser sheet intensity variations. Shan and Dimotakis [33] investigated the mixing behavior of an unconfined transverse jet between jet Reynolds numbers of 1000 and 20000 at a velocity ratio of 10 for all cases. They used rhodamine-6G chloride in concentrations between $1.4 \times 10^{-6} \mathrm{~mol} / \mathrm{L}$ and $1.4 \times 10^{-5}$ mol/L. coupled with a frequency-doubled Nd:YAG laser $(\lambda=532 \mathrm{~nm})$. This was partially due to work by Karasso and Mungal [34] that found that sodium fluorescein had a non-linear response when excited by an Nd:YAG laser. While studying the suitability of rhodamine for measurements, Shan et al. [35] found the fluorescence response was linearly proportional to dye concentration to within the concentration uncertainty for three different laser intensities. For each run they recorded a background image to remove the effect of ambient light on data collection and also operated in a darkened lab with an optical filter in front of the CCD camera. These considerations were made for the current effort. 


\section{Experimental Design}

\subsection{Historical Considerations}

From the aforementioned background literature, several variables stand out as being significant for study in the current effort and may be categorized as either geometric or flow-related. The most significant geometric variables include the diameter ratio $(d / D)$ and the number of jets (synonymous with spacing). Geometric variables expected to have second-order influence on flowfield mixing are injection port shape and flow injection angle, each of which affects penetration and pressure drop across the jet. The most significant flow variables include the velocity ratio (first-order influence) and density ratio (second-order influence). These two parameters may also be combined to form the momentum flux ratio (J). In order to limit the design space to a reasonable size, diameter ratio, number of jets, and velocity ratio were initially chosen as test parameters.

Figures 18 and 19 identify points tested in the referenced literature for blowing ratio (square root of momentum flux) and diameter ratio, each plotted against the number of jets. The blowing ratio was used to incorporate differences in density ratio between experiments. Figure 18 indicates there is a good spread in the data coverage among velocity ratios, ranging from approximately 4-100 (two orders of magnitude). However, there is a notable gap between one and six jets at low blowing ratios (below 4.5). Figure 19 displays the same experimental cases, instead plotting diameter ratio versus number of jets. There is a lack of data for low numbers of jets $(\mathrm{n}<8)$ above a diameter ratio of about 0.13 . 


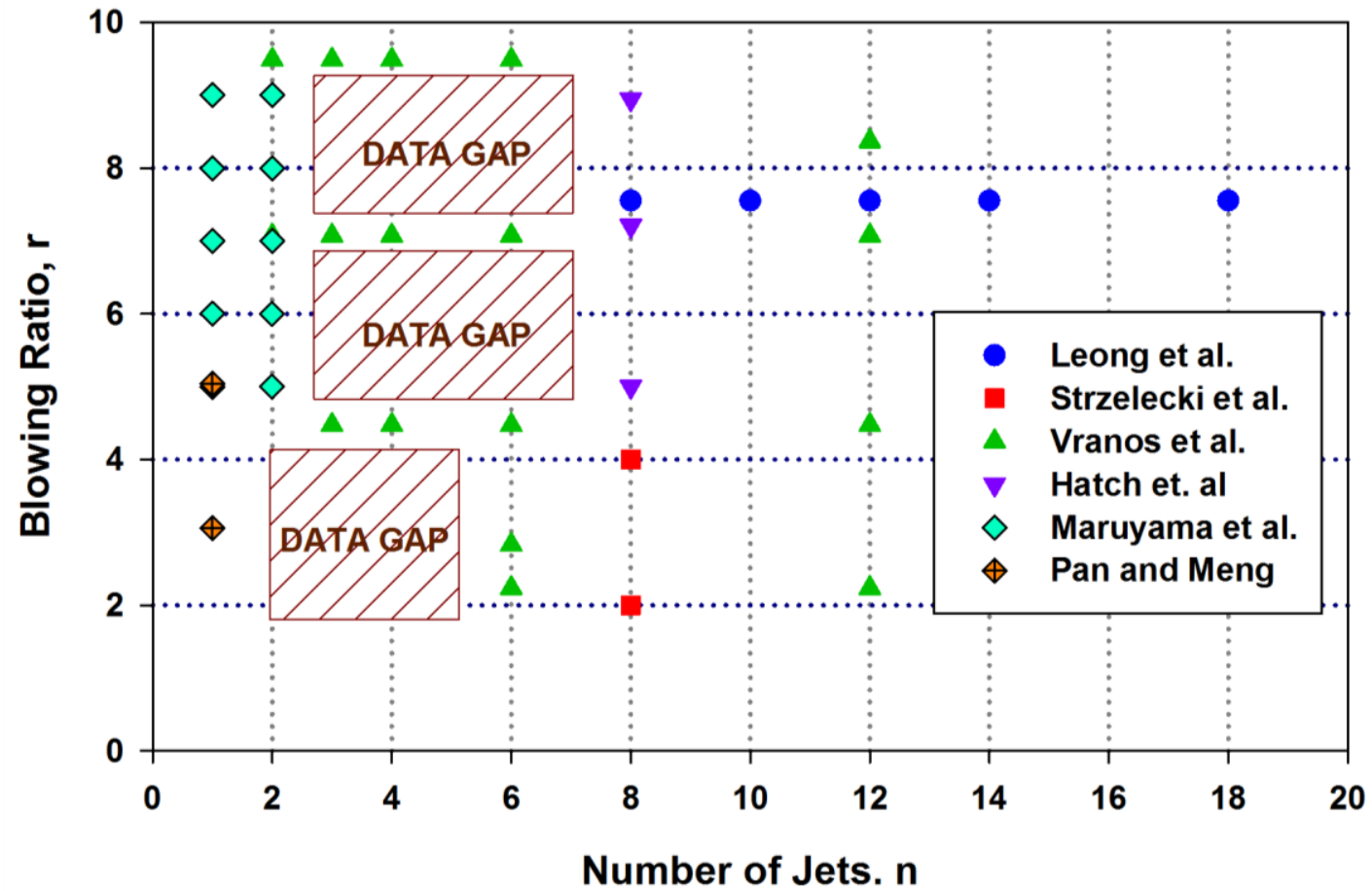

Figure 18: Blowing ratio versus number of jets for historical data.

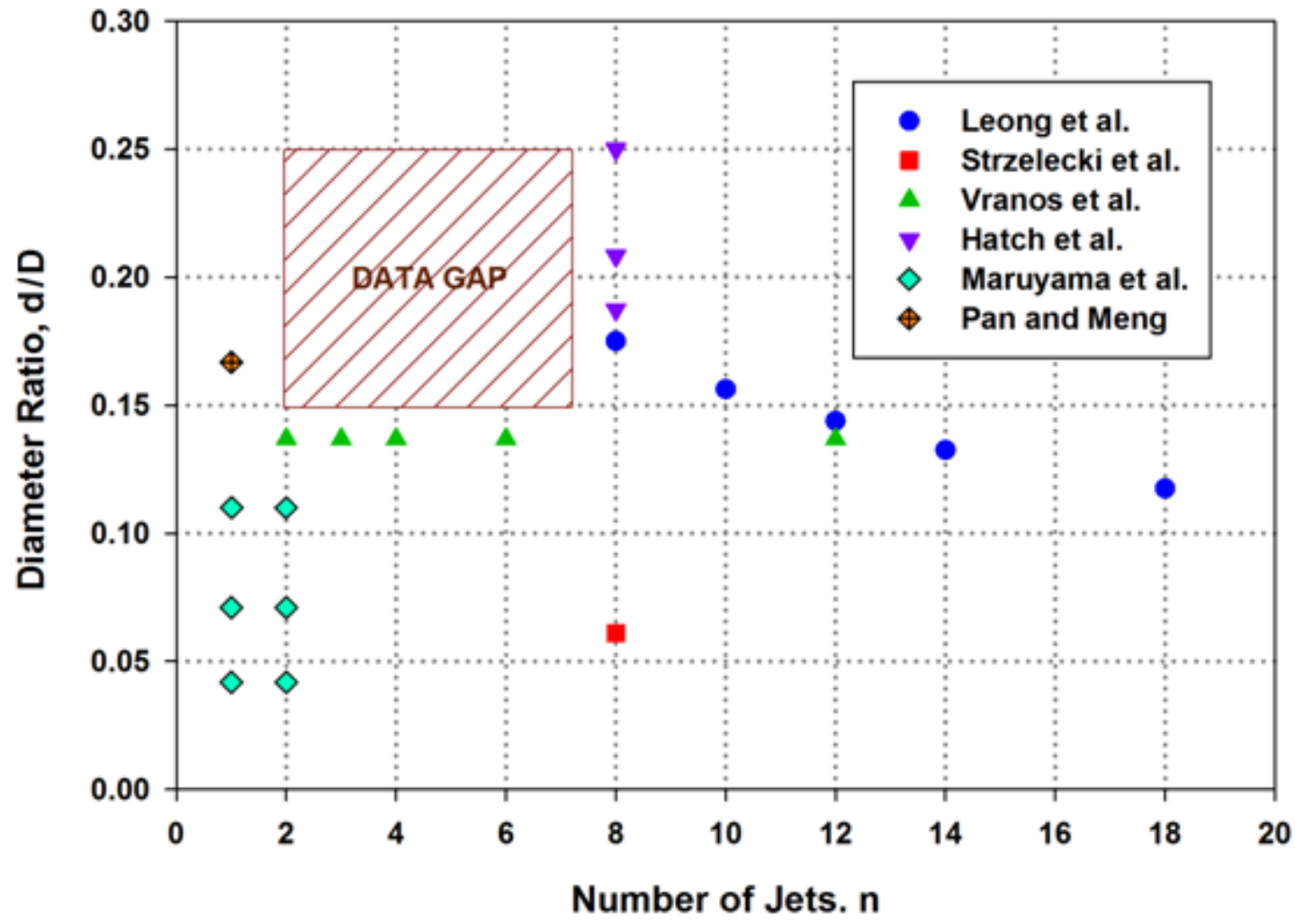

Figure 19: Diameter ratio versus number of jets for historical data. 
These two figures indicate that more data are necessary to more completely evaluate the mixing performance of configurations involving few multiple jets, specifically at lower blowing ratios and higher diameter ratios. Since the momentum flux ratio is equal to the blowing ratio squared, this correlates to momentum fluxes between approximately 10 and 50. Diameter ratio data exists for small numbers of jets up to approximately 0.14 . In order to provide some overlap, 0.12 was chosen as the bottom end of diameter ratios, with 0.21 as the top end. Since single and dual jets are fairly well documented in the literature, a single jet was chosen as the bottom end for testing to provide verification of mixing measurements. The six jet configuration was chosen as the upper end, as a static apparatus with six jets could accommodate one, two, three, or six jets while maintaining symmetry. This also corresponds to the expected deviation from Holdeman scaling $(n=2 \pi)$ given by equation 11 . A derivation of this expected deviation may be found in Appendix C.

In order to provide the most robust definition of mixing, the relative unmixedness defined by Vranos [24] (Equation 14) was chosen as the primary objective measure of mixing. In order provide a direct comparison with literature (most notably Sroka and Forney [20]), the second moment of concentration was chosen as a secondary measure to quantify mixing variation at the measurement plane. The second moment of concentration can be defined as

$$
M=\frac{1}{A} \int_{A} \frac{(C-\bar{C})^{2}}{\bar{C}^{2}} d A
$$

where $c$ is the time-averaged local concentration and $\bar{c}$ is the spatial mean of these time averages. The equation for $\bar{c}$ is

$$
\bar{C}=\frac{1}{A} \int_{A} C d A=C_{o}\left(\frac{n Q_{j}}{n Q_{j}+Q_{c}}\right)
$$

where $A$ is the cross-sectional area, $C_{o}$ is the jet fluid concentration, $n$ is the number of jets, $Q_{j}$ is the jet flow rate and $Q_{c}$ is the primary flow rate. This measure can be directly compared to the single-jet 
correlation from Sroka and Forney [20] (Equation 6) as the momentum ratio can be easily calculated from the momentum flux ratio and the diameter ratio.

\subsection{Apparatus Considerations}

The Air Force Research Laboratory at Edwards AFB previously operated a water flow loop for experiments related to heat transfer. In order to minimize costs and time for facility design, some of this apparatus was reused and incorporated into the current experiment. With the design limits laid out in Section 3.1, the facility required modifications and additions to achieve the appropriate velocity ratios, diameter ratios, and numbers of jets.

The existing apparatus uses an electronically-controlled pump to deliver between 10 and 60 GPM to the test section at moderate head rise (10-50 psig). The water flow is delivered to the test section and may be re-circulated to the storage tank. The intended use of PLIF as a flow measurement technique inherently requires seeding of the jet flow with a tracer to measure mixture fraction distributions. If the dye-mixed fluid from the current experiment were to pass back into the storage tank for the primary flow, it would then contain increasing amounts of tracer as testing continued, biasing the results. Separate tanks are therefore required to achieve independent control for the primary and jet injection flow rates. Additionally this setup requires a means of catching the wastewater from the test section.

With material and machining costs in mind, a static test section with interchangeable inserts provided the most flexibility for changing diameter ratio and number of jets between experiments. This would allow mass production of jet inserts, with only the internal diameters varying between different sets. Using this framework, initial concepts were drawn using a CAD package. Figures 20 and 21 detail cutaways of the preliminary concepts for the injection block and interchangeable inserts. These inserts 
would then be fed by flexible hoses attached to a manifold distributing the jet fluid. This concept had several issues, such as ease of access to the inserts in proximity of the flange, optical access for the laser downstream of the injection location, and equal distribution of the jet fluid in the manifold to ensure each line had equal flow rates.

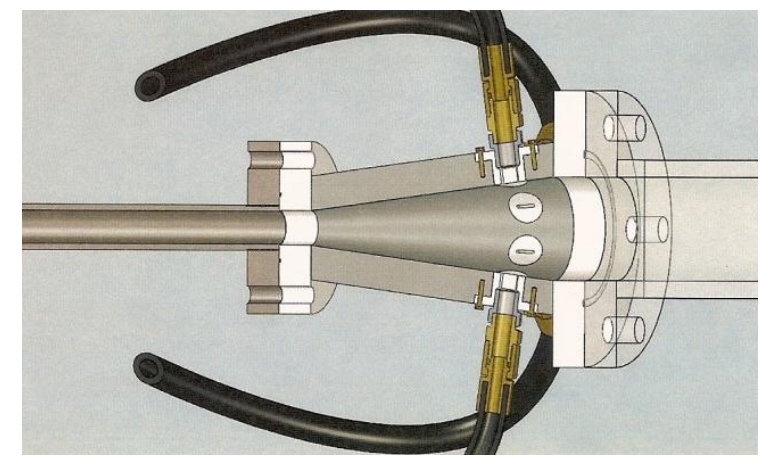

Figure 20: Cut-away of test section concept. Concept for jet inserts show slot jets (not used).

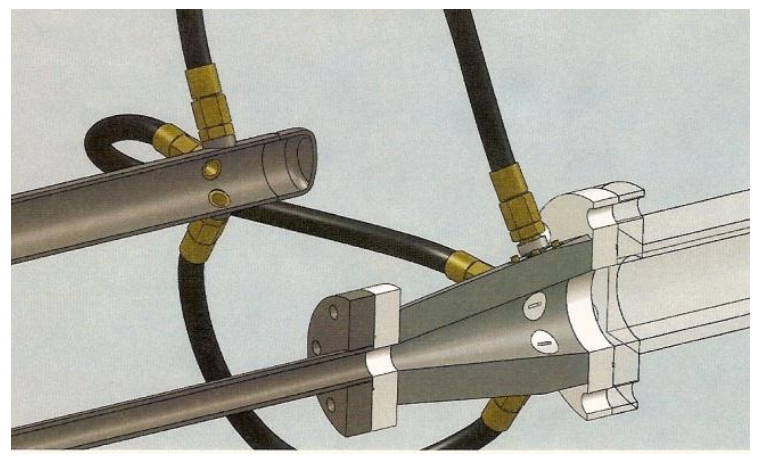

Figure 21: Test section concept with jet fluid manifold. Concept for jet inserts show slot jets (not used).

The concern of optical access was addressed by redesigning the structure holding the injection ports to reduce the distance between the injection port and the optical test section. The manifold was adjusted from a radial distribution (as seen in figure 21) to a linear distribution (figure 22) with all six lines at the same elevation to avoid pressure changes due to height. The pressure distribution in the manifold was analyzed with a CFD model and estimated to produce a maximum variation of 0.07 psi between the first and last injection lines. This was sufficient to produce a flow rate variation of less than one percent.

In order to determine the flow rate through each line, a flow metering device was necessary. For liquid water, the cavitating venturi serves this purpose well. A cavitating venturi is a specially designed nozzle that induces cavitation in a liquid to constrict the liquid flow area such that changes in downstream pressure have no effect on delivered mass flow rate. Flow rate then becomes only a function of the inlet pressure, $\mathrm{P}_{1}$. Traditional design criterion specifies that as long as the static pressure ratio $\mathrm{P}_{2} / \mathrm{P}_{1}$ across the cavitating venturi remains below approximately 0.85 , cavitation will occur. The anticipated test section static pressures were between 23 and 33 psia depending on flow conditions. To provide sufficient 
operating margin, 94 psia was chosen as the design specification. Seven venturis were purchased, with the six closest calibrated in terms of calibration being used in operation.

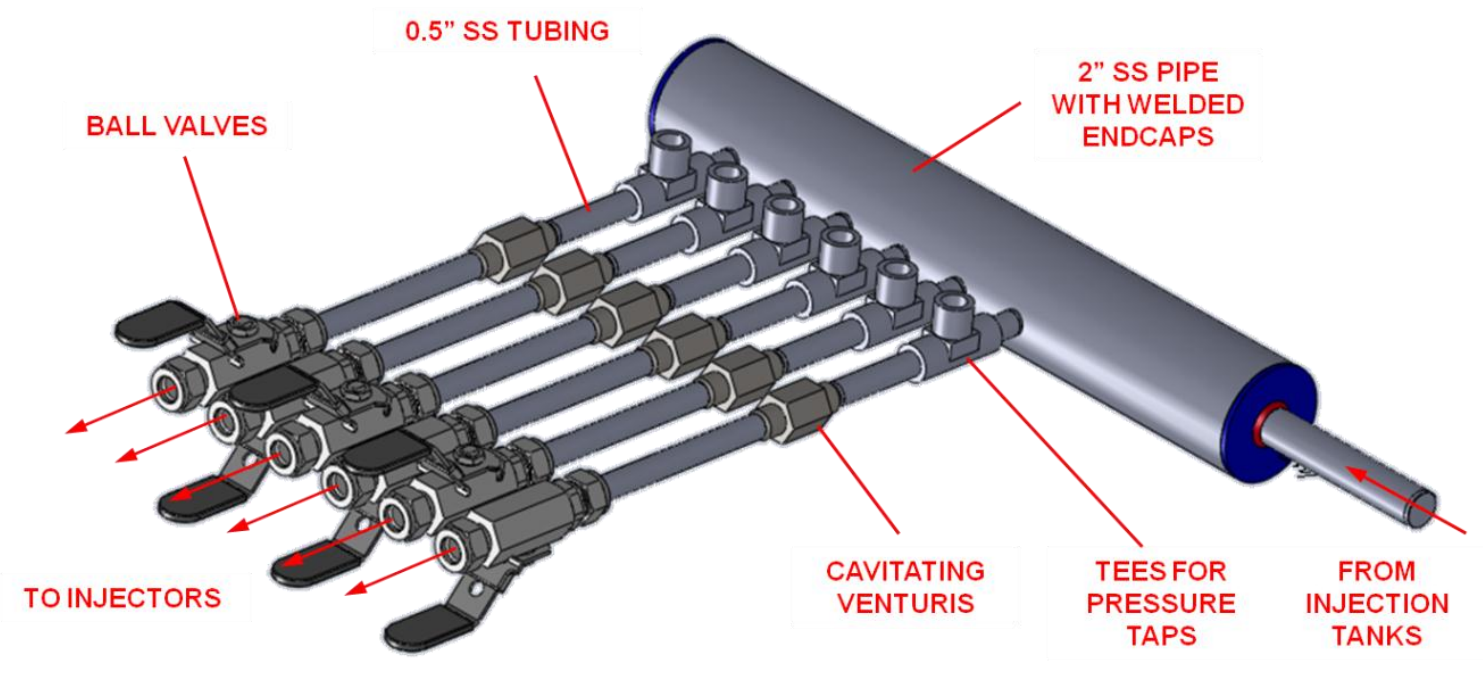

Figure 22: Linear-distribution injection manifold.

The two options for jet fluid delivery to the manifold were pump-driven or pressure-driven. Since pump-driven systems tend to have some transient pressure fluctuations, a pressure-driven system was selected and integrated easily with the facility gaseous nitrogen supply as the pressurant.

\subsection{Operating Limits and Test Points}

One disadvantage posed by a cavitating venturi flow meter is the limited range of operability offered in terms of flow rate capability. The venturis were calibrated over a 20 psi range, corresponding to range of flow rates of 0.67 to 0.75 per venturi. In essence, the designed system is fixed in jet flow rate and core diameter while maintaining variability in core flow rate and jet diameter. A trade study was necessary to determine if the system design was capable of meeting test point criteria as laid out in Section 3.1. The definition of momentum flux ratio is

$$
J=\frac{\rho_{j} v_{j}^{2}}{\rho_{c} v_{c}^{2}}
$$


Since this experiment uses water as the working fluid for both primary and jet flows, the density ratio may be taken to be unity and neglected. Substituting the continuity equation, rearranging to solve for velocity, squaring and cancelling terms, one obtains

$$
J=\dot{m}_{j}^{2} \dot{m}_{c}^{-2}\left(\frac{d}{D}\right)^{-4}
$$

In equation 19, three distinct parts appear. The first is the term "fixed" by the mass flow rate of a single jet, the second is the variable term adjustable by the mass flow rate of the primary flow and the third is the variable term adjustable by the diameter ratio. By simply multiplying by the relative areas and again cancelling terms, equation 17 is modified into the momentum ratio such that

$$
\beta=\dot{m}_{j}^{2} \dot{m}_{c}^{-2}\left(\frac{d}{D}\right)^{-2}
$$

From preliminary testing of the facility, it was found that using only an existing bypass valve and the pump settings, primary flow rates between 5 and 30 GPM could be obtained. Using an orifice to backpressure the test section delivery line, flow rates as low as $2.0 \mathrm{GPM}$ could be achieved. A MATLAB script was written to generate parameter operating space contour plots with the relative ranges of obtainable flow. The results are shown in Figs. 23-26. Each of the horizontal lines represents a diameter ratio to be tested. It was concluded that with minor modifications including an orifice plate, the flow loop sufficiently met operability criteria to achieve desired goals for momentum flux ratio and momentum ratio. 


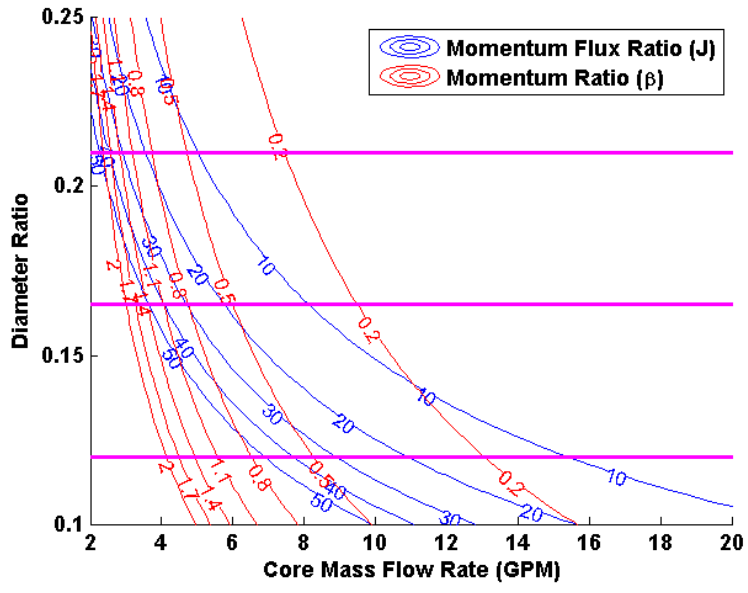

Figure 23: Design space for $n=1$.

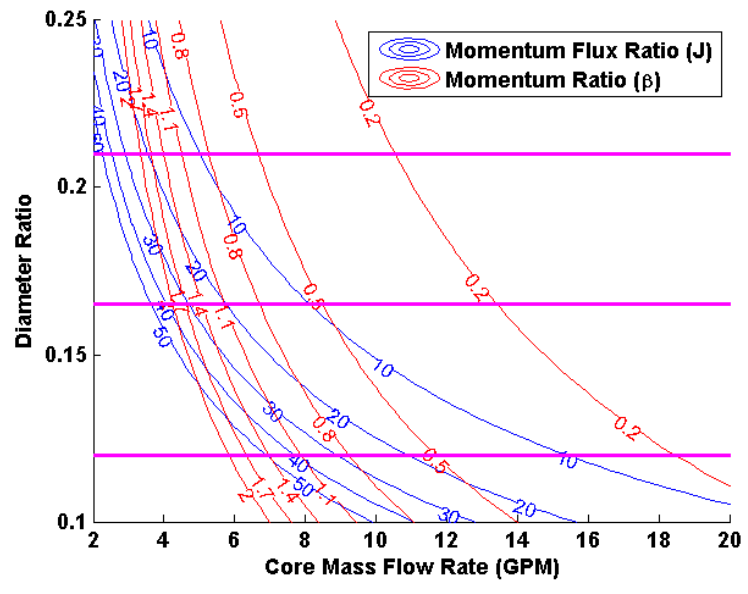

Figure 24: Design space for $n=2$.

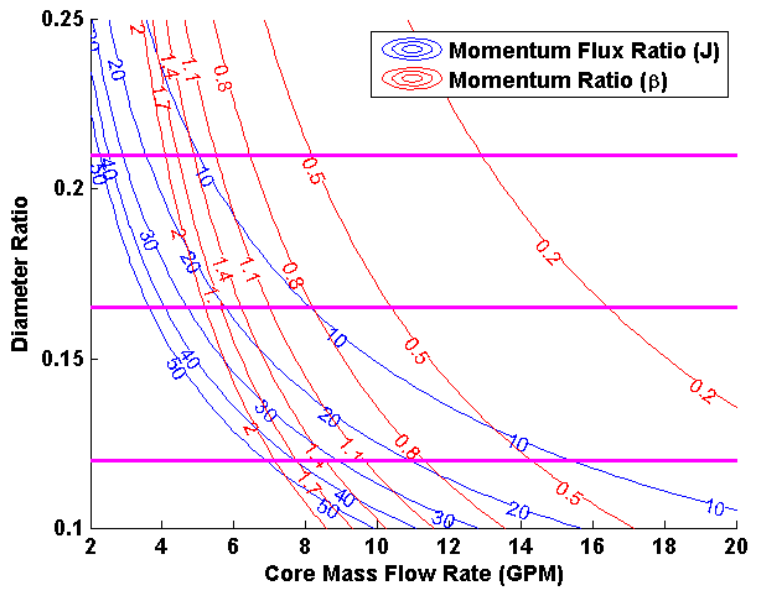

Figure 25: Design space for $n=3$.

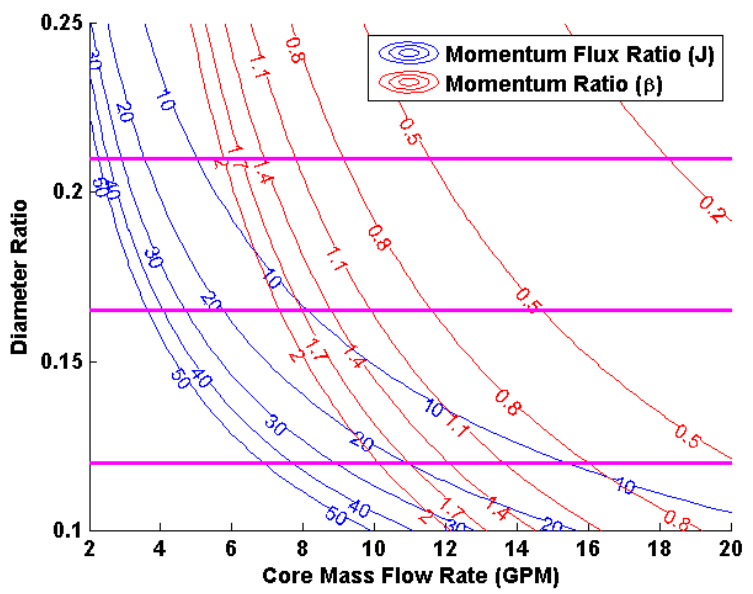

Figure 26: Design space for $n=6$.

Once this design space had been identified, Forliti [36] proposed and derived a scaling parameter that accounts for both drag and entrainment as mechanisms for momentum transfer from the crossflow to the jets. This parameter, B, is described in more detail in Reference $36 . \mathrm{B}$ is derived as

$$
B=\frac{J}{\frac{2 C_{D}}{\pi}+c_{e j} J^{1 / 2}}
$$


where $C_{D}$ is the drag coefficient on the jet column and $c_{e j}$ is the entrainment coefficient. Mashayek et al. [15] identified the drag coefficient for a single jet-in-crossflow to be approximately 1.7. Ricou and Spaulding [37] identified the entrainment coefficient for a single jet-in-crossflow to be approximately 0.32. These values were used in all calculations of B. Once B was selected to be the scaling parameter of choice, values of $\mathrm{B}$ were calculated such that the test values focused around $B(d / D)=1$ as suggested by Forliti. Increments of 0.25 were selected, providing a total range from 0.25-1.75 given seven test points. This resulted in values of momentum flux ratio between 3 and 47, which fall within the system capabilities described in Figs. 23-26. 


\section{Apparatus}

\subsection{Water Flow Loop}

The Project Themis water flow test facility is built upon legacy hardware at AFRL Area 1-14. The original apparatus was designed for heat transfer studies. The apparatus has been modified to deliver a range of flow rates appropriate for the current effort. Figure 27 illustrates a general schematic of the flow loop modified for the current experiment.

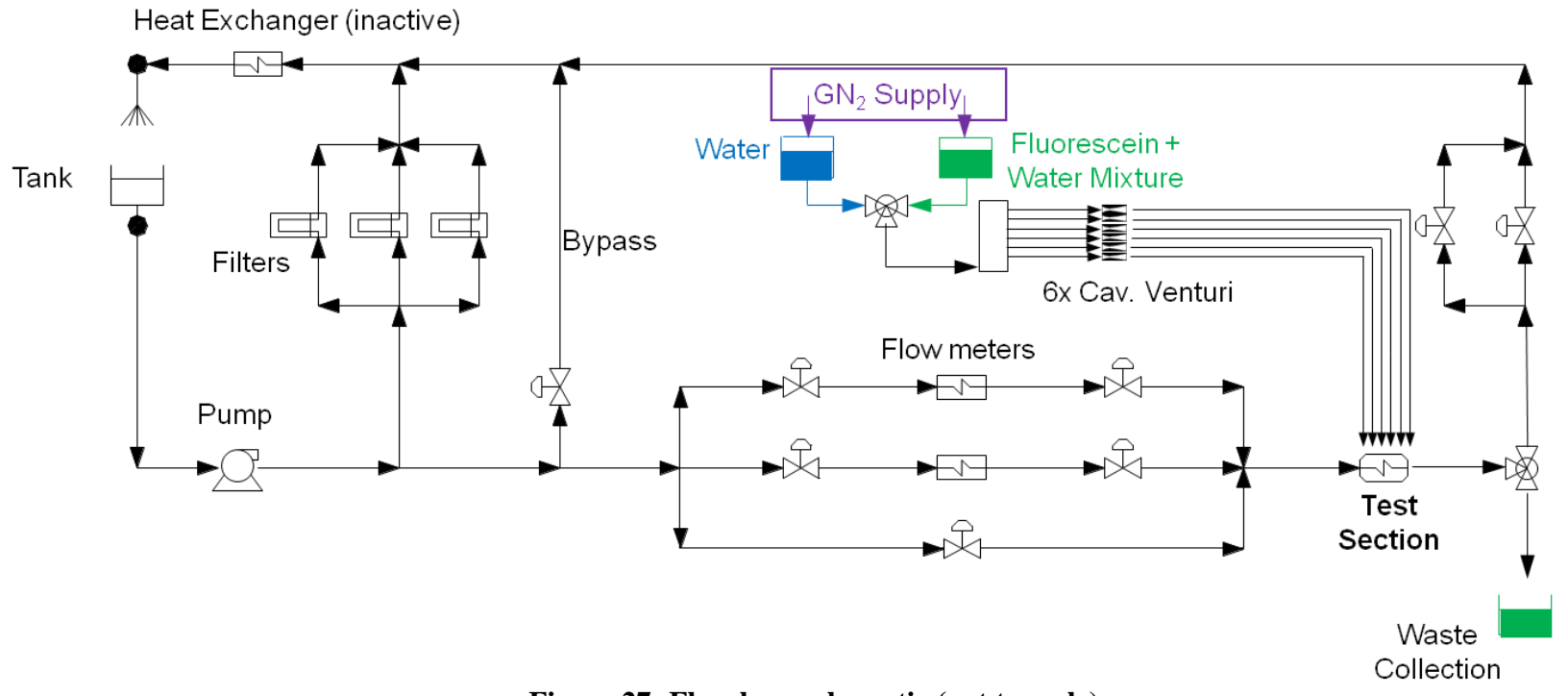

Figure 27: Flow loop schematic (not to scale).

\subsubsection{Primary Flow Loop}

The core flow originates from a sixty gallon tank open to atmosphere and is connected to an Ebara A3U32-200 pump, capable of delivering 10-120 GPM and a pressure head of 10-100 psi. The pump is controlled using a variable-speed electronic controller with a range of approximately 500-1800 rpm. Typical settings range from 525 to $1200 \mathrm{rpm}$. Figure 28 shows the general arrangement of the pump and primary flow run tank. 


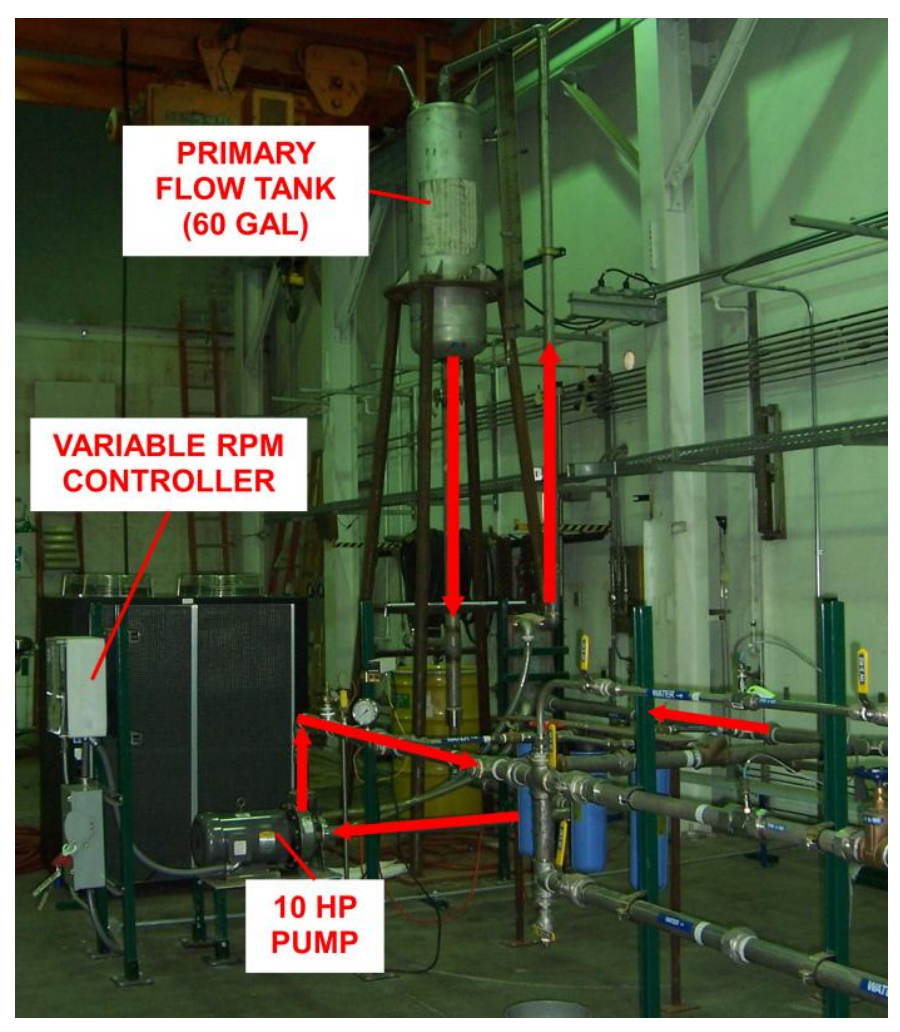

Figure 28: Primary flow loop run tank and pump.

Since the test matrix calls for flow rates below the range of the pump, a bypass valve and interchangeable orifice plates are used to reduce the flow delivered to the test section to the required range. The flow goes through a Flow Technology Inc. model FT-16A50-LB turbine flowmeter, calibrated using a bucket and stopwatch over the range of flow rates of interest (2-16 GPM). Two interchangeable sharp-edge orifices of different size were used to increase the hydraulic resistance of the test section. The small orifice is used for the low flow regime (2-6 GPM) and the large orifice is used for the high flow regime (6-16 GPM). After passing through the appropriate orifice, the flow goes through a turbulent runup section with an ID of 1.592". With a total L/D of approximately 48, the inflow condition of the test section is expected to be fully-developed turbulent pipe flow. This assumption was verified using Laser Doppler velocimetry measurements at various Reynolds numbers and compared to data from literature. Further details may be found in Appendix B. Figures 29 and 30 depict the upstream piping and turbulent run-up sections respectively. 


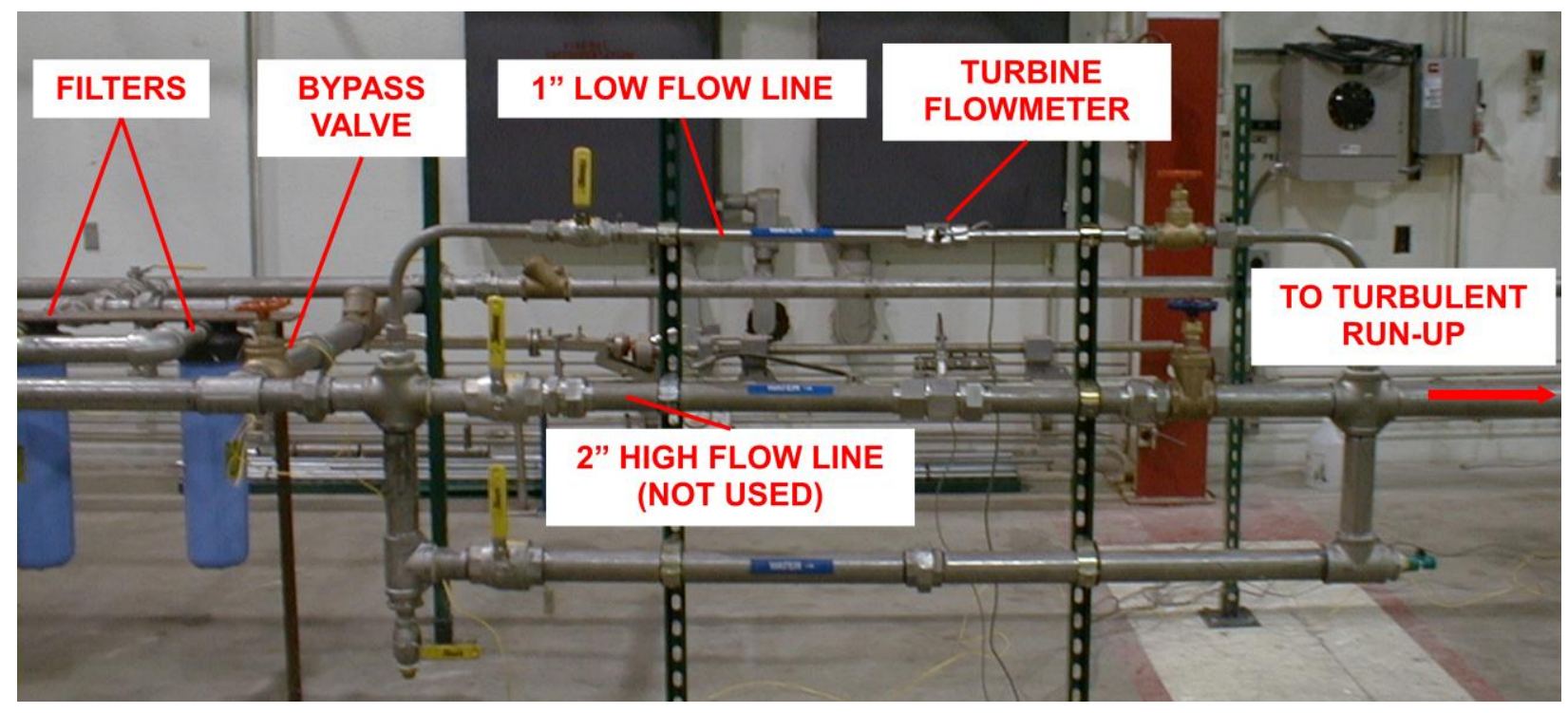

Figure 29: Bypass and upstream piping.

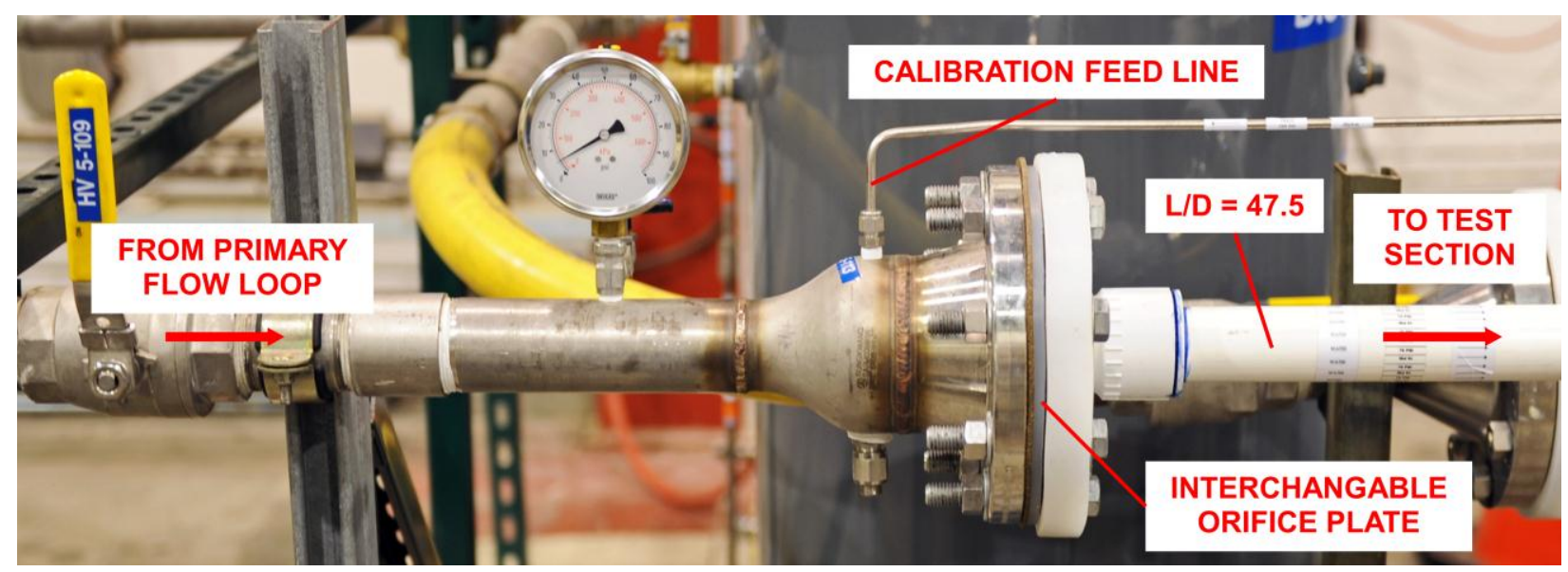

Figure 30: Orifice plate, calibration feed line and turbulent run-up.

\subsubsection{Injection System}

The injection system consists of two 200 psi rated pressurized tanks connected to the injection manifold (see figure 27). One tank contains water for testing and set-up, the other contains dyed water for data collection. Each tank has a capacity of sixty gallons and is outfitted with a sight glass to check fluid level when depressurized. Under test conditions, the tanks are pressurized up to 94 psia from the facility gaseous nitrogen lines. Figure 31 shows the nitrogen pressure lines and the injection run tanks. 


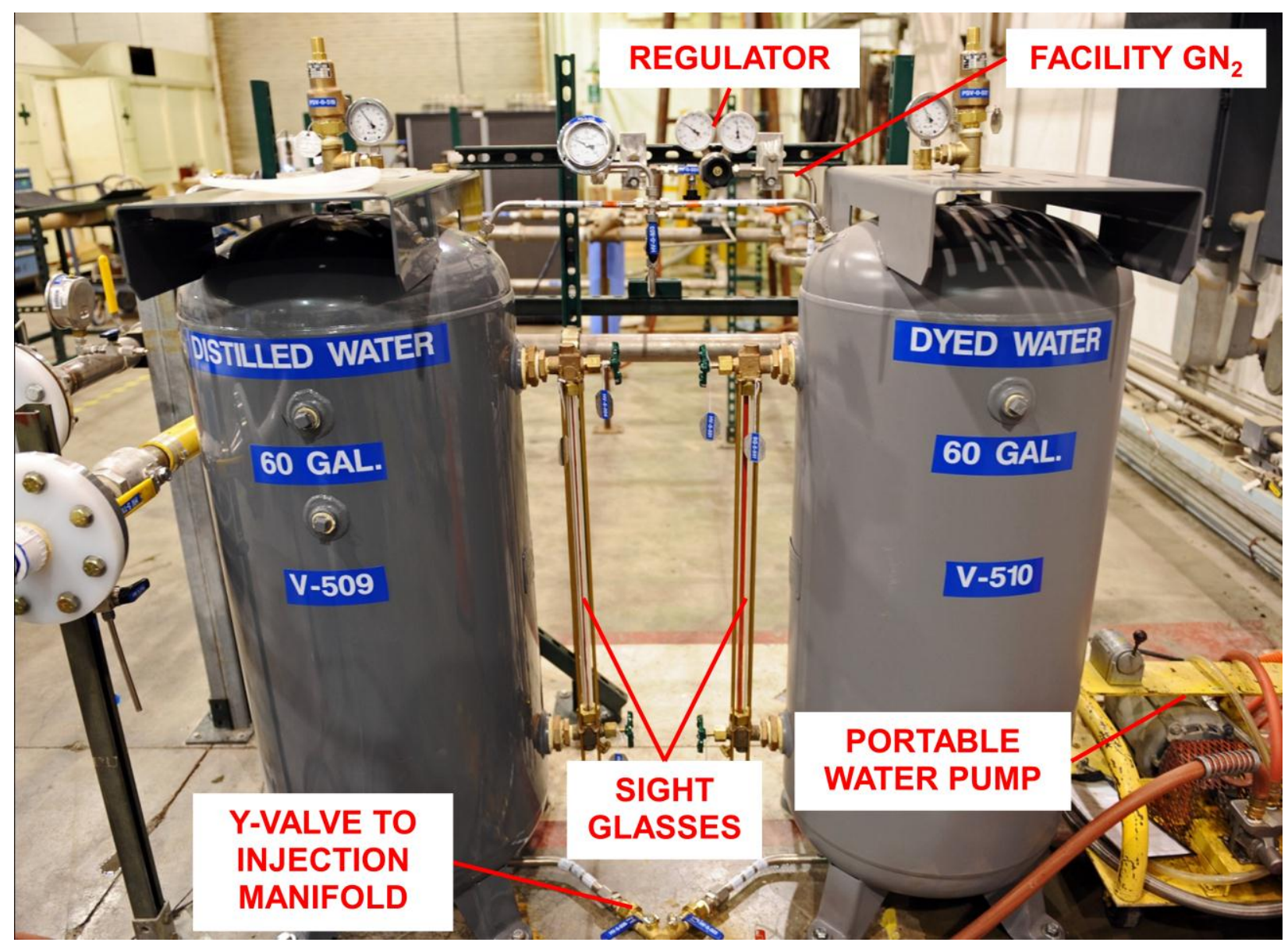

Figure 31: Jet fluid injection tanks.

Figure 32 illustrates the injection manifold and the six injection lines leading to the test section.

The manifold is constructed of 2" steel pipe with one inflow line, six outflow lines, and a drain. The static pressure variation between each line is less than 0.07 psi. This translates to a volumetric flow rate difference of less than $1 \%$ between any two lines. The flows are metered by Flowmaxx cavitating venturis, each with a nominal flow rate of 0.7 GPM at a design inlet pressure of 94 psia. The static pressures are monitored by two absolute transducers and four differential transducers. Although use of differential meters to read subsequent line pressures does allow for error propagation, existing hardware had to be used within budgetary constraints. Additionally, the calibration coefficients for all the meters were well within acceptable limits and pressure readings were nearly constant. Calibration curves for the transducers (pressure versus voltage), the venturi nozzles (flow rate versus pressure) and the main loop 
flow meter (flow rate versus frequency) were included in the facility LabView program and used to calculate momentum flux values for each test run. Section 5.2 describes the calibration process in more depth.

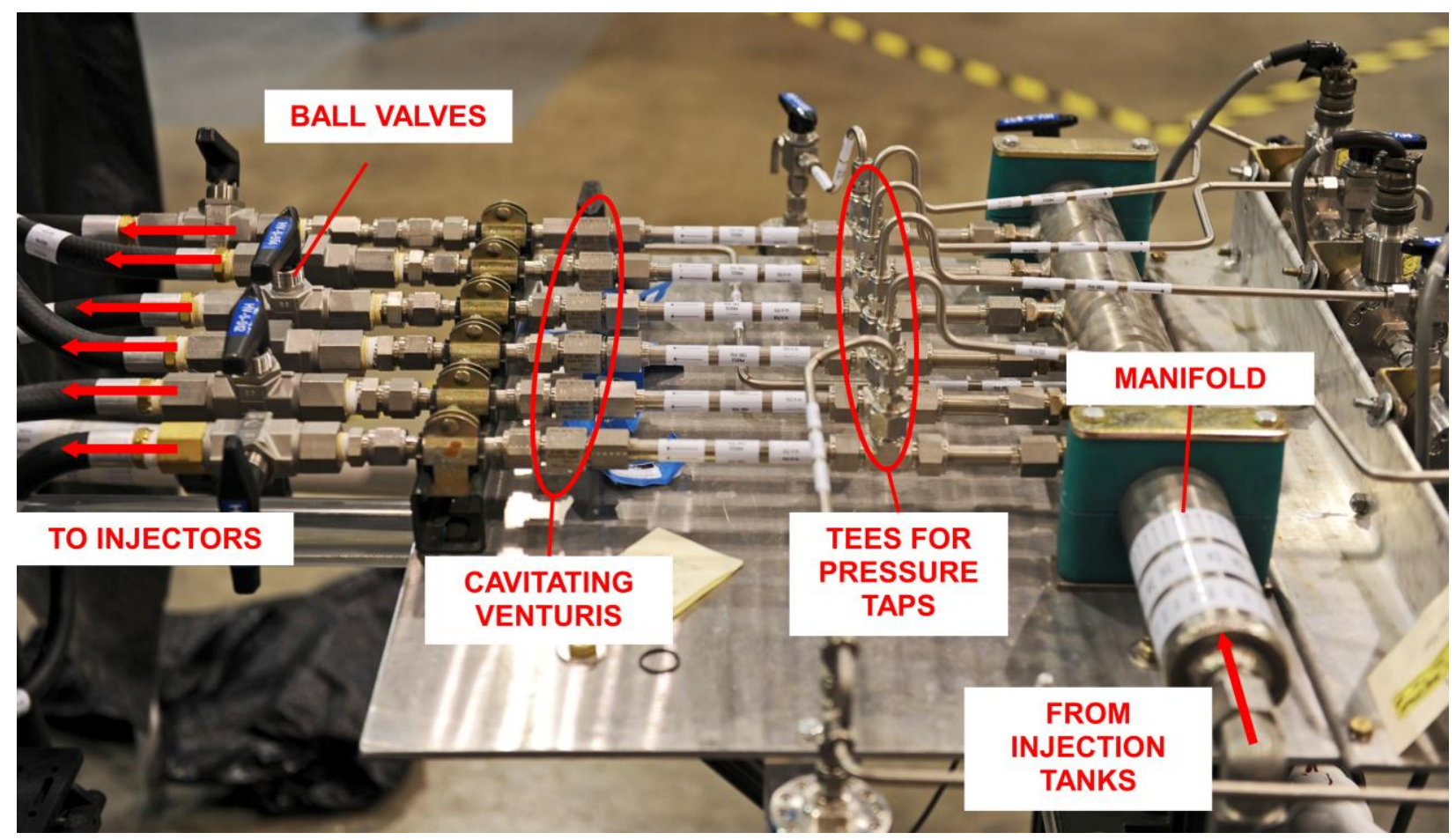

Figure 32: Injection manifold and hardware.

As shown in figure 33, the test section consists of a hexagonal nylon block machined to accommodate jet inserts. Figure 34 shows a representative insert. The jet inserts are machined from 6061 grade aluminum and have 0.5 inch adapters welded to allow connection to flex hosing from the injection lines. Different sets of inserts exist for different diameter ratios; results will be shown for a diameter ratio of 0.12. An optical access window was placed upstream of the test section to allow for LDV measurements of inflow velocity profiles. However, due to opacity this proved ineffectual. Velocity profiles were instead taken slightly downstream of the injection location in the test section. 


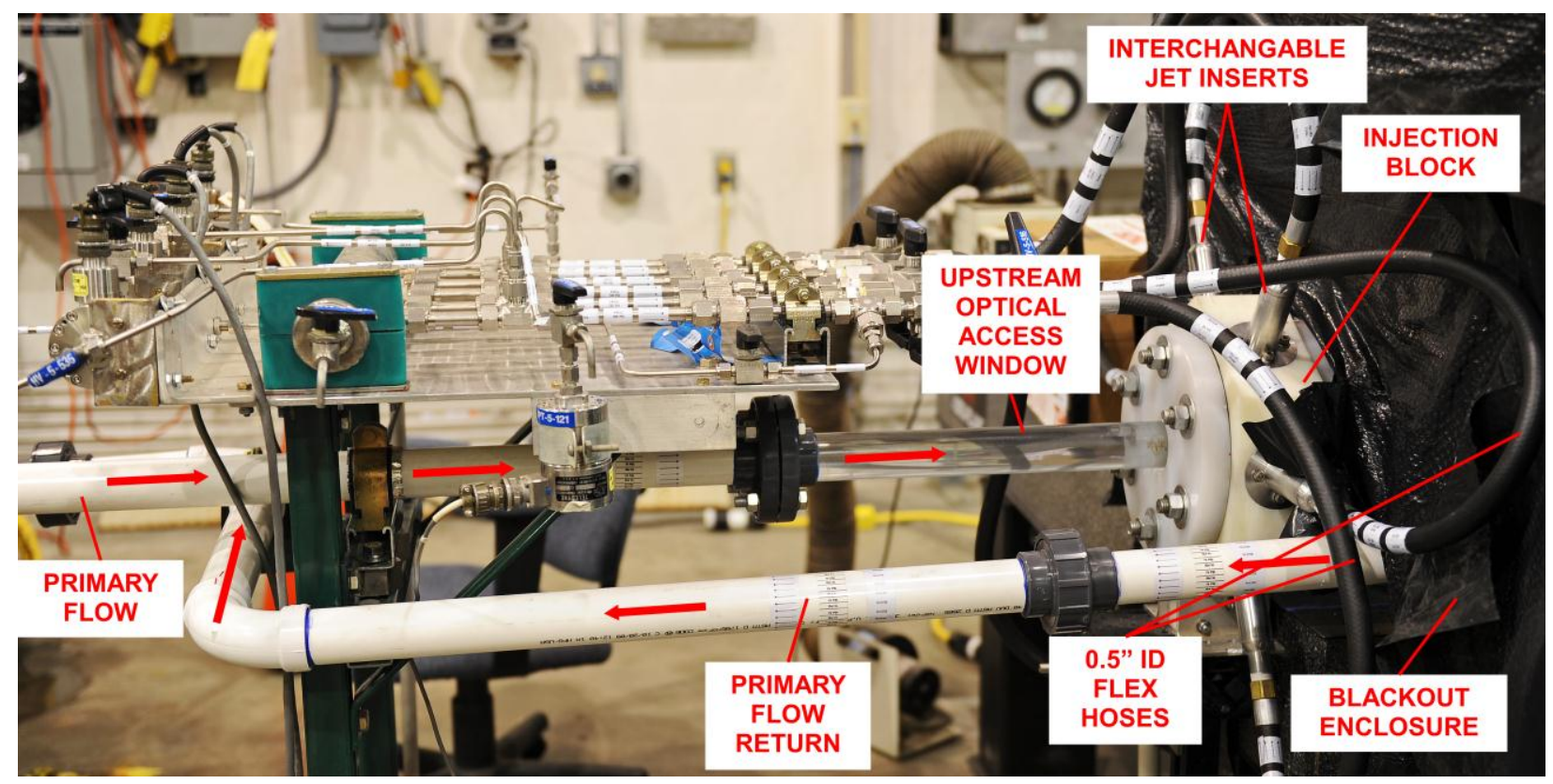

Figure 33: Primary flow loop piping and injection block.

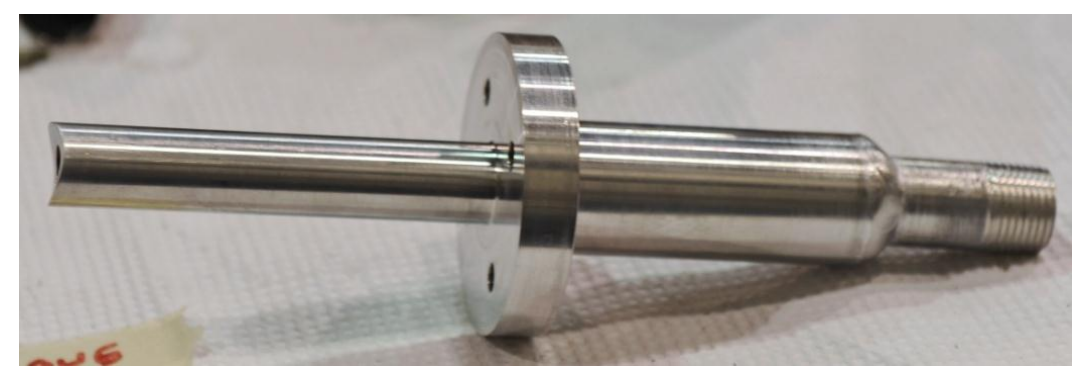

Figure 34: Close-up of jet insert $(d / D=0.165)$.

\subsubsection{Test Section}

An acrylic section (2" square external, 1.592" internal diameter) is affixed to the outflow of the injection block, allowing laser access between $\mathrm{x} / \mathrm{D}=2.0$ and $\mathrm{x} / \mathrm{D}=4.5$. All PLIF measurements for the current effort have been taken at $\mathrm{x} / \mathrm{D}=3.0$; additional axial locations may be investigated in future studies. A three inch polypropylene tee is affixed to the exit of the test section with an acrylic viewing window cut into the end. This allows the camera optical access to the flow cross-section as seen in figure 35. After passing through the test section the water is directed through a tee which is outfitted with a viewing window for camera access. The water passes through a 1.5" diverting three-way PVC valve and 
is either recirculated back to the supply tank for flow loop setup using regular water or delivered to a fifty-five gallon drum during testing. Visible in figure 36, a 1.5" PVC ball valve is used to provide back pressure to the test section and control the primary flow rate.

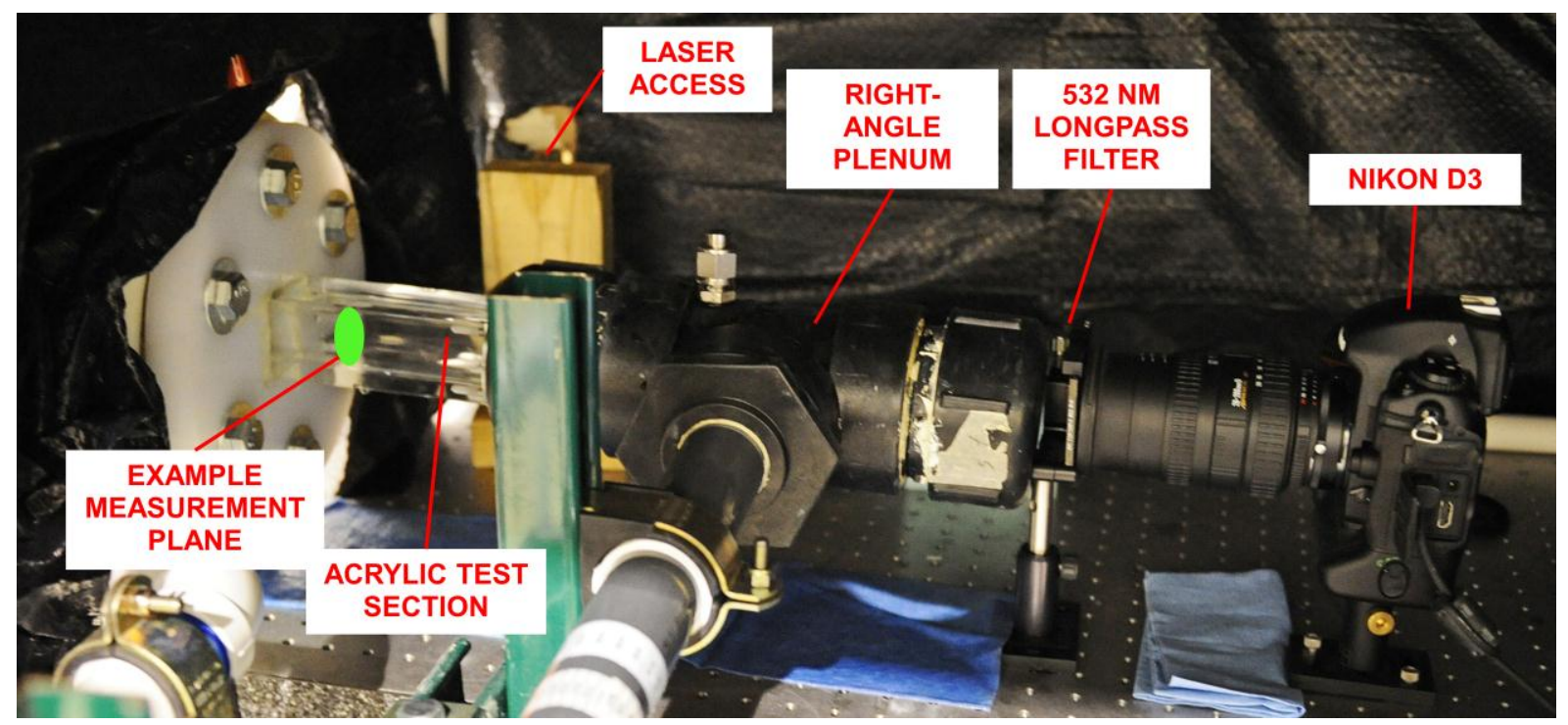

Figure 35: Test section with optical equipment

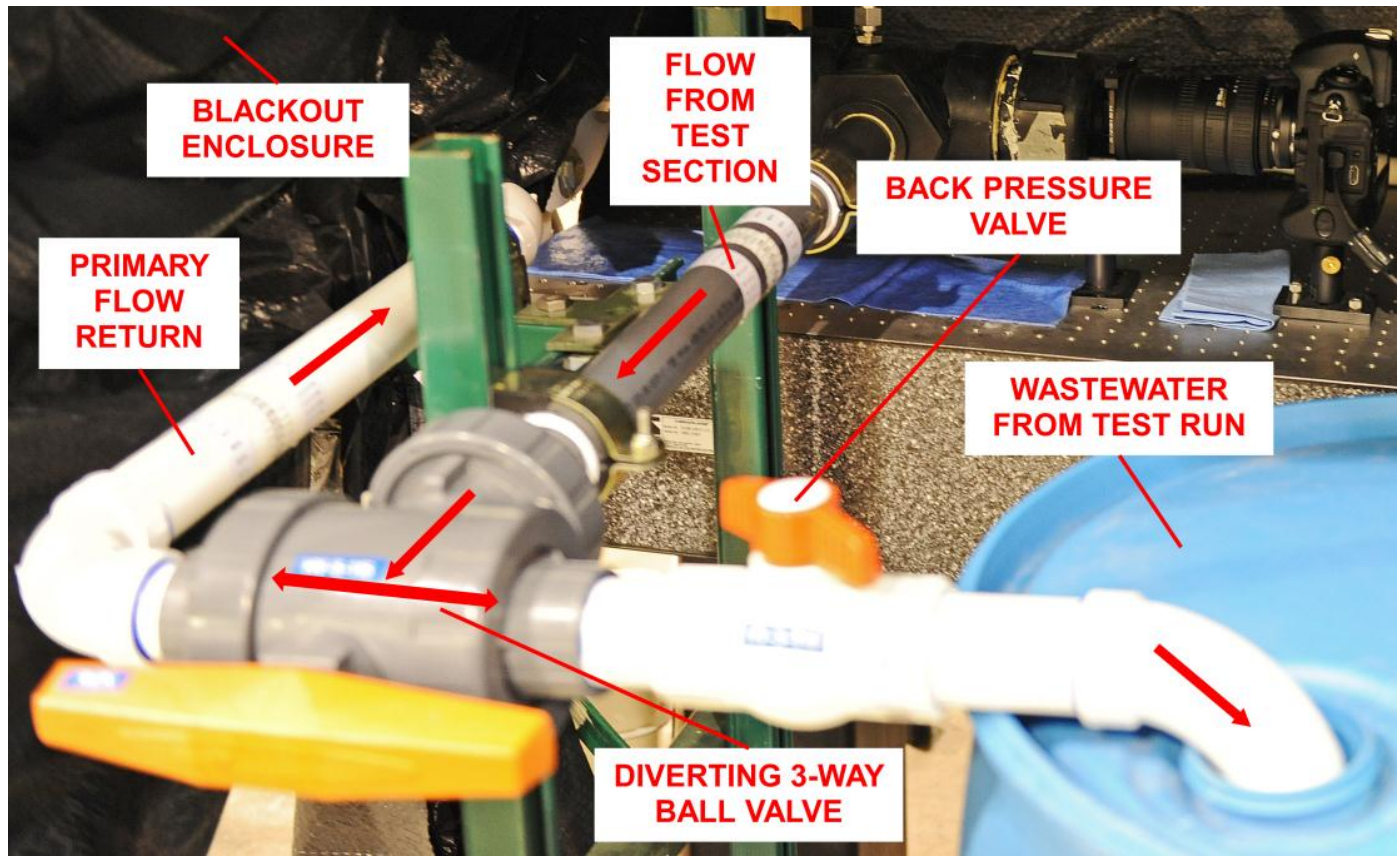

Figure 36: Test section outflow piping 


\subsection{Optical Components}

The selected optical configuration is bi-lateral, using a laser perpendicular to the flow and a camera parallel to it. An Ion laser Technology ILT 5500A argon-ion laser operating at reduced power (300 milliwatts) was filtered using a dichroic mirror that filters the $515 \mathrm{~nm}$ signal and passes the lower wavelength components. This is necessary to avoid confounding of the laser signal at the expected fluorescence bands. The signal is then focused using a spherical lens ( $\mathrm{f}=200 \mathrm{~mm}$ ) and spread into a sheet using a pair of cylindrical lenses $(f=-25.4 \mathrm{~mm})$ to illuminate the dyed water. The profile of the beam was Gaussian and the central peak (maximum outward to two standard deviations) was spread to approximately two inches, matching the outside of the acrylic test section. Figure 37 shows optical elements used to shape the laser sheet (dichroic mirror not pictured).

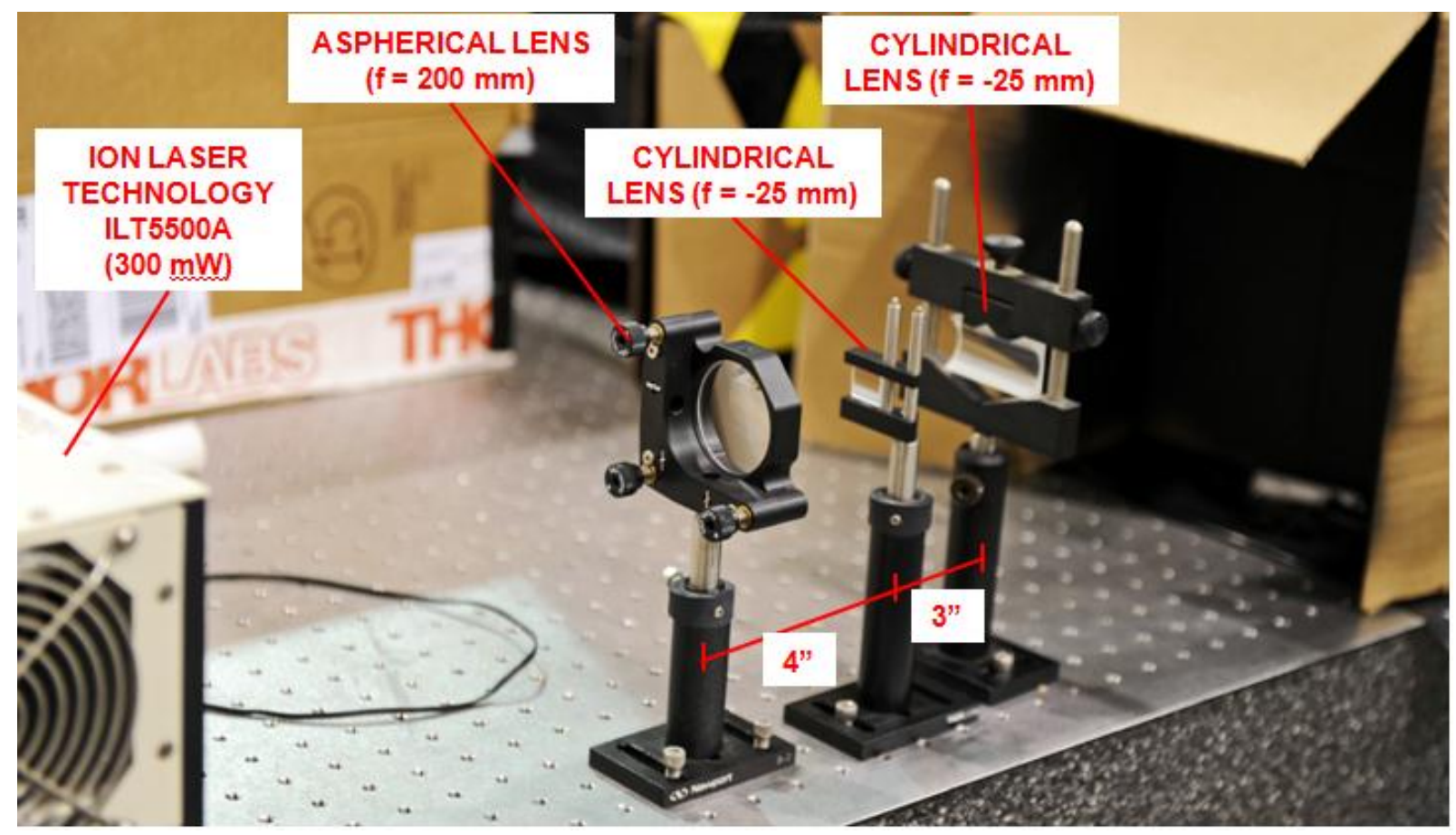

Figure 37: Optical shaping components

After flowing through the test section the waste water goes through a tee outfitted with another acrylic window for the data collection camera (Nikon D3) as shown in figure 35 . The camera was set to take images using an uncompressed 14-bit TIF format. All light was filtered using a $505 \mathrm{~nm}$ longpass filter, eliminating shorter wavelengths from the laser signal from all data collected. 


\section{$5 \quad$ Validation and Calibration}

\subsection{Validation of Analysis Code}

Due to the large number of data to be processed, an efficient code structure was implemented using MATLAB. This code structure called a primary function to perform image evaluation within specified boundaries and deliver the mean and second moment of concentration. This function was used repetitively in a script to process image data for all test cases. A modified version was used to process calibration images and use this data to normalize raw images.

In order to proceed with confidence, a validation of the code was performed. An arbitrary distribution was created, solved analytically and compared to results generated by the code. This distribution represents a simple stratified flowfield exhibiting various concentrations in a radial step function as shown in figure 38 . The red curve denotes the analysis boundary.

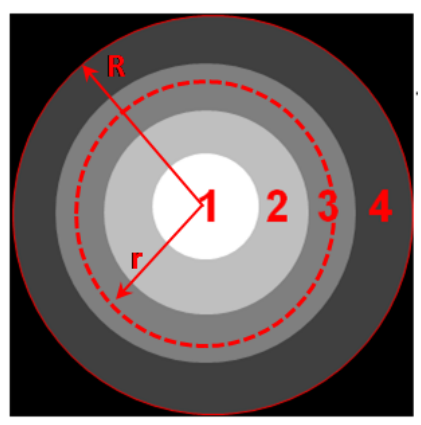

\begin{tabular}{|c|c|c|}
\hline Region & Radius & Concentration [mol/L] \\
\hline 1 & $\mathrm{r} / \mathrm{R}<0.25$ & $1.00 \mathrm{E}-07$ \\
\hline 2 & $0.25<\mathrm{r} / \mathrm{R}<0.5$ & $4.64 \mathrm{E}-08$ \\
\hline 3 & $0.5<\mathrm{r} / \mathrm{R}<0.75$ & $2.15 \mathrm{E}-08$ \\
\hline 4 & $0.75<\mathrm{r} / \mathrm{R}<1$ & $1.00 \mathrm{E}-08$ \\
\hline
\end{tabular}

Figure 38: Radial step function used to validate image analysis code.

The code identifies pixels in a face-centered algorithm, such that a pixel is counted if the center of its area is within a specified radial distance. If not, the pixel is discounted. The code then calculates the second moment based on equation 13. Since the images analyzed are discrete data, the integral equations may be converted to summations. Since the pixel areas are all equal, the second moment may be represented as 


$$
M=\sqrt{\frac{1}{m} \sum_{i=1}^{m} \frac{\left(c_{i}-\bar{c}\right)^{2}}{\bar{c}^{2}}}
$$

using an if-else structure to discriminate the boundary.

As the image size is increased in the digital space, the code more accurately calculates the true mean and second moment of the input data. Raw images were taken with the test section comprising approximately 400x400 pixels. Figures 39 and 40 illustrate the code convergence and percent errors respectively.

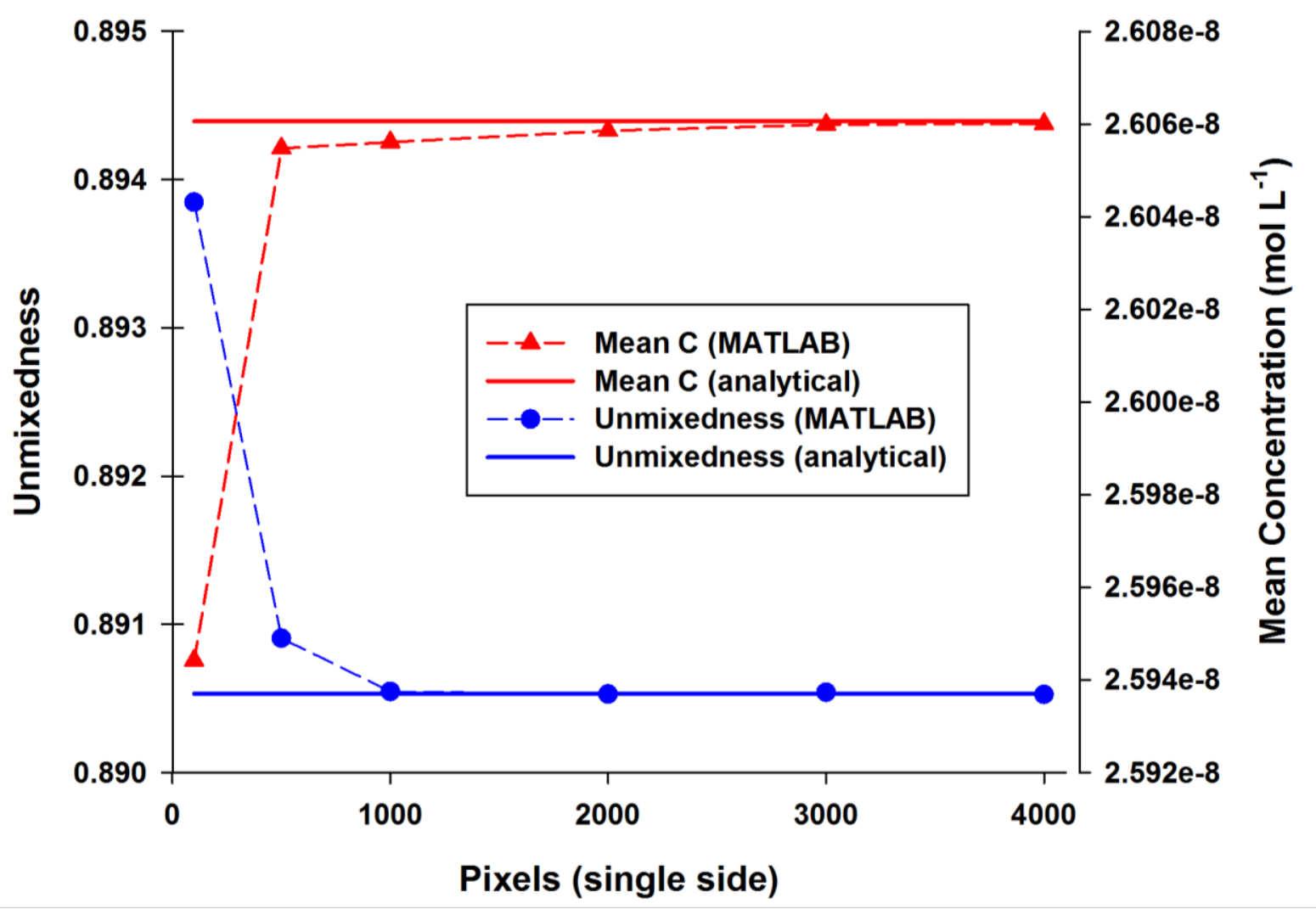

Figure 39: Convergence of code output parameters using test distribution. 


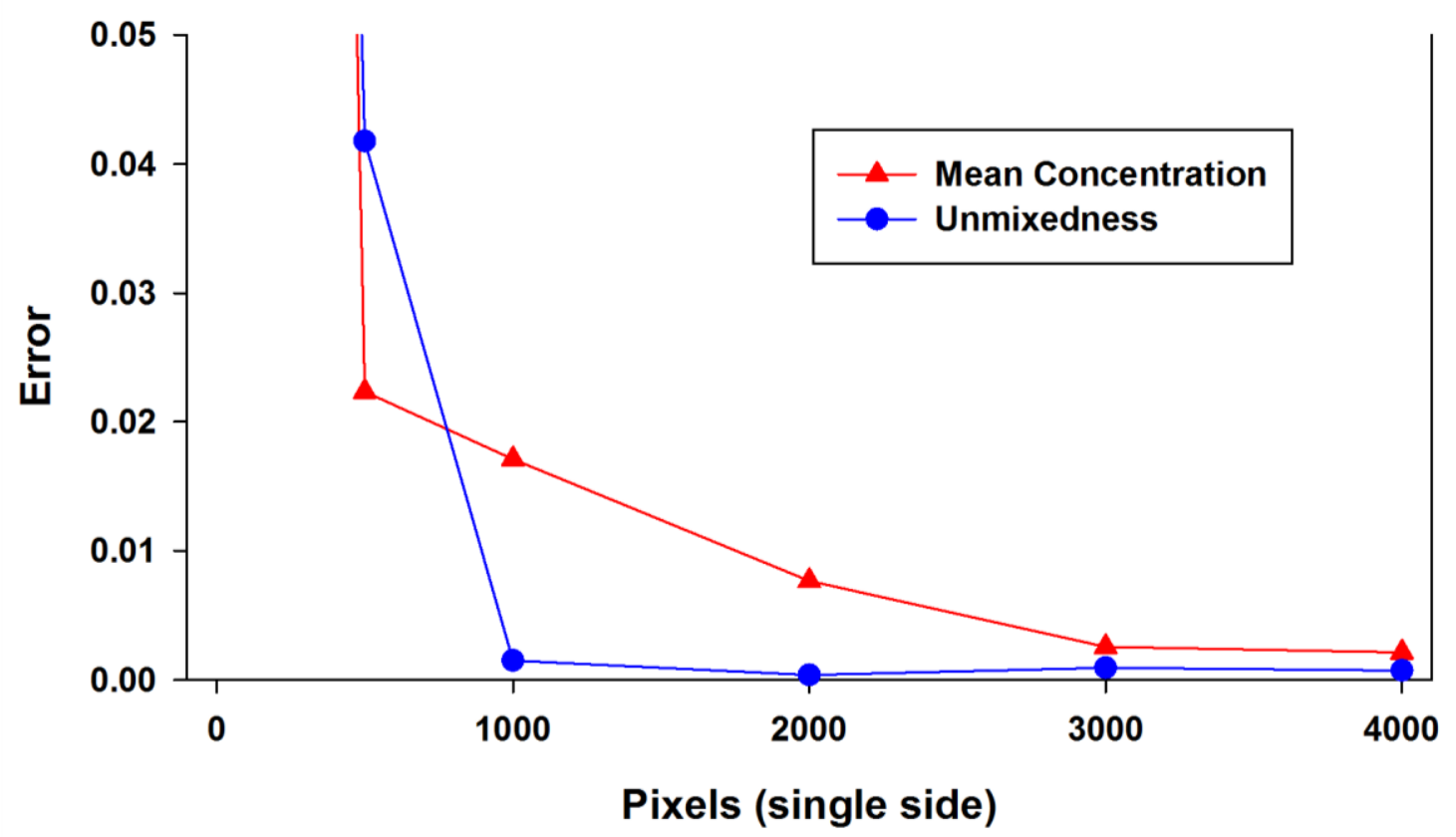

Figure 40: Analysis code output error as a function of image size.

\subsection{Calibration}

Calibration was conducted on a daily basis by taking a darkfield reference image and diverting various levels of jet fluid upstream through the feed line visible in figure 30 . This allowed the full length of the turbulent run-up section to be used to mix the jet fluid, producing an assumed homogenous mixture for each condition of jet-to-total flow rate. This produced several calibration images each day, allowing a correlation to be calculated for each pixel within the analysis area. This minimizes any variations in laser sheet illumination on the data collected. Figure 41 plots the linear $\mathrm{R}^{2}$ value for each pixel for the calibration data used with single-jet and two-jet data. Figure 42 plots the $\mathrm{R}^{2}$ for three jets and figure 43 for six jets. Due to reflection and refraction at the interface between the acrylic test section and the flow channel, the $\mathrm{R}^{2}$ in this region is low. To compensate, the analysis area was reduced by approximately two percent in radius, removing all pixels with an $\mathrm{R}^{2}$ less than 0.99 . 


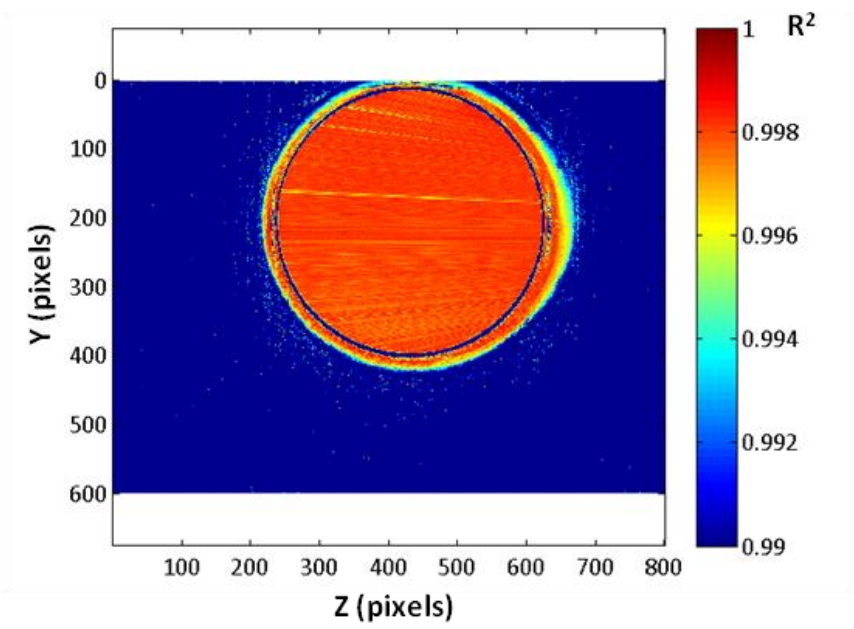

Figure 41: $\mathbf{R}^{2}$ values for calibration data used on one and two jet data.

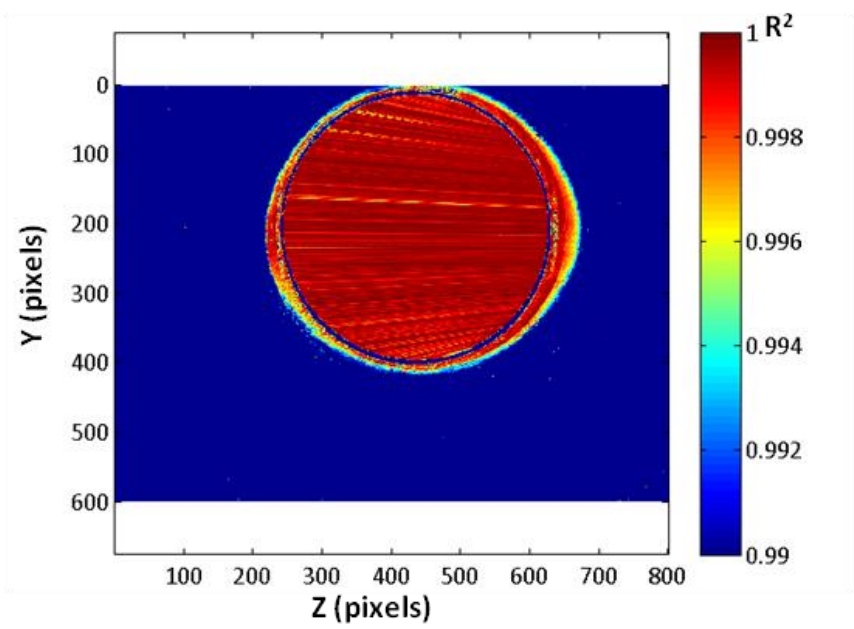

Figure 42: $\mathbf{R}^{2}$ values for calibration data used on three jets.

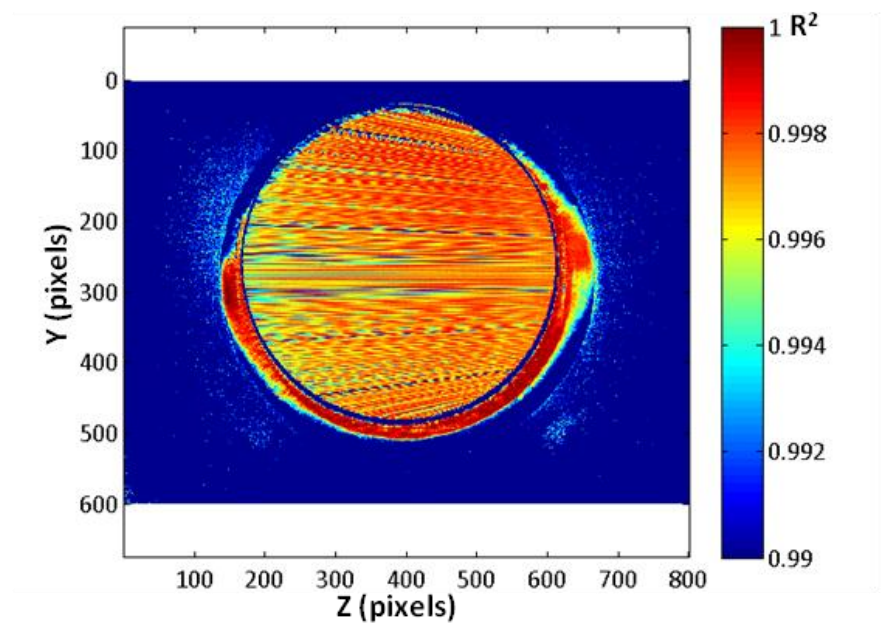

Figure 43: $R^{2}$ values for calibration data used on six jets. 


\section{Results}

\subsection{Mixing Performance as a Function of $B(d / D)$}

Due to facility complications and scheduling constraints, the test matrix had to be reduced. Consequently, repeatable data could only be collected for only the smallest diameter ratio (0.12). All data presented in this and subsequent sections are for this diameter ratio.

The non-dimensional mixing parameter B developed by Forliti has created more universal scaling among single jet data, both for jet trajectories and scalar mixing. Investigation of this parameter is worthwhile for the current data set, as similar values for $B(d / D)$ were calculated at each jet number. Figure 44 illustrates the unmixedness data as a function of $B(d / D)$.

The trends are very interesting to note for these data. A single jet (shown in red) behaves much the way literature describes - a local minimum exists at a value lower than $B(d / D)=1$, mixing degrades for some a range of $B(d / D)$ then begins to improve again as $B(d / D)$ is increased beyond one. This is due to a nearly centered jet having greater area to spread before reaching the mixing plane (corresponding to the local minimum). When the jet momentum is barely large enough to impact the adjacent wall (Fig. 45), the impulse is not great enough to cause small-scale breakup of the jet. Instead, the jet instead coheres to the opposite wall (Fig. 46) and then bifurcates and follows the contours of the wall as it progresses downstream (Fig. 47). Eventually the momentum is increased to a point where the bifurcated jet streams impact each other on the same side of the primary flow path as they originated, colliding once again to induce breakup and mixing with crossflow fluid (Fig. 48). 


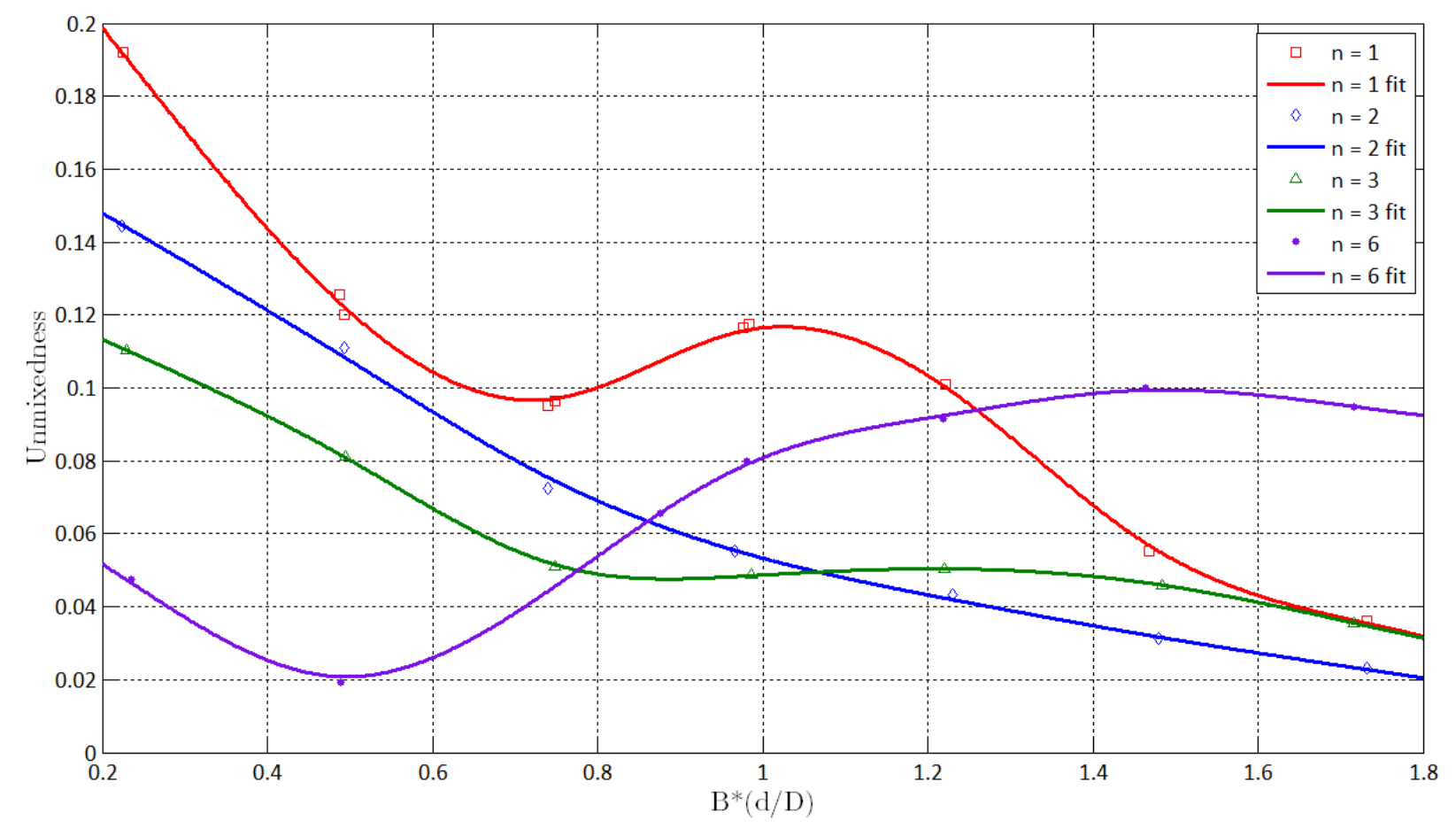

Figure 44: Unmixedness as a function of $B(d / D)$.

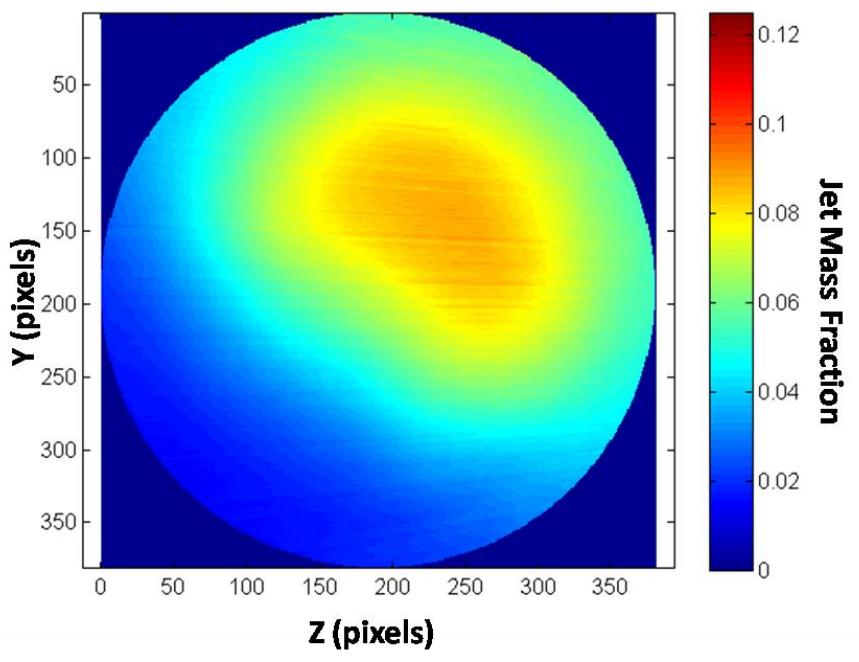

Figure 45: Jet mass fraction for $n=1, B(d / D)=0.75$ 


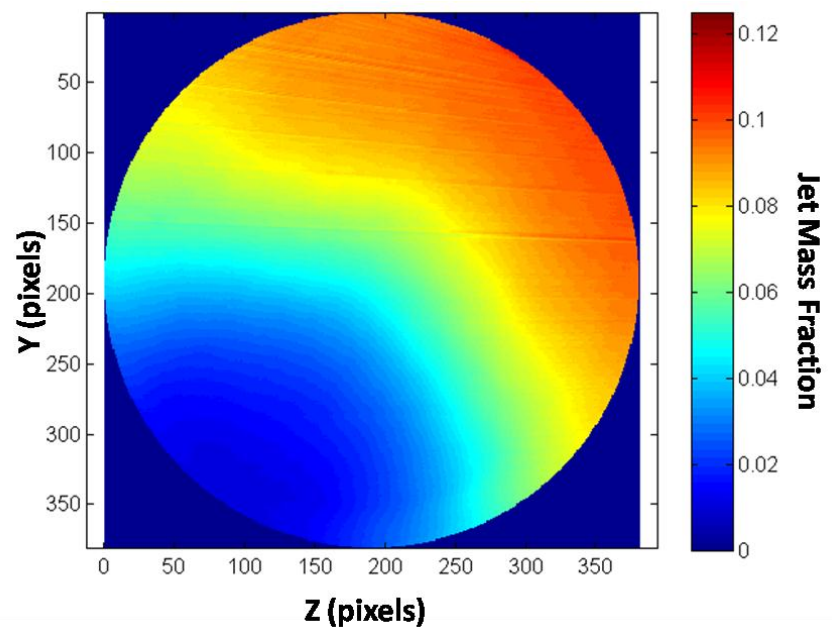

Figure 46: Jet mass fraction for $n=1, B(d / D)=1.00$

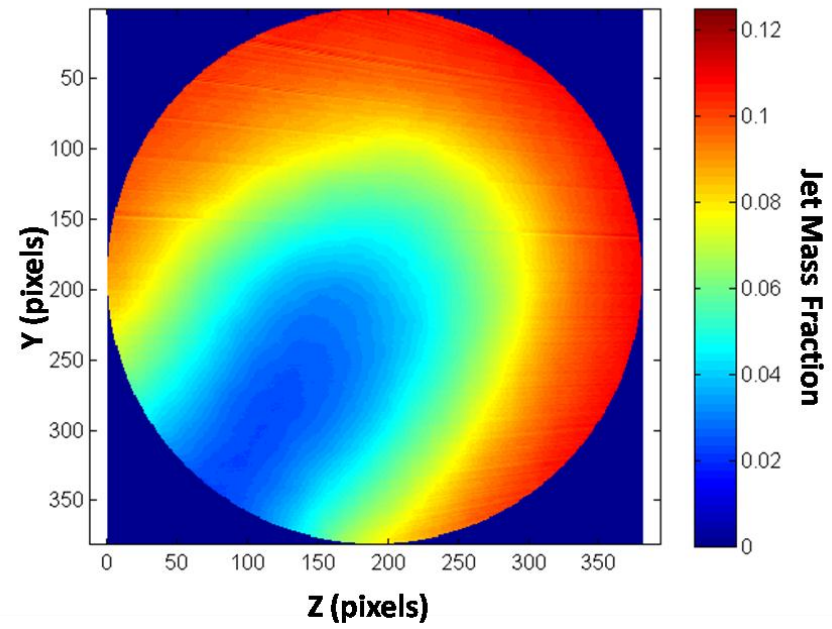

Figure 47: Jet mass fraction for $n=1, B(d / D)=1.25$

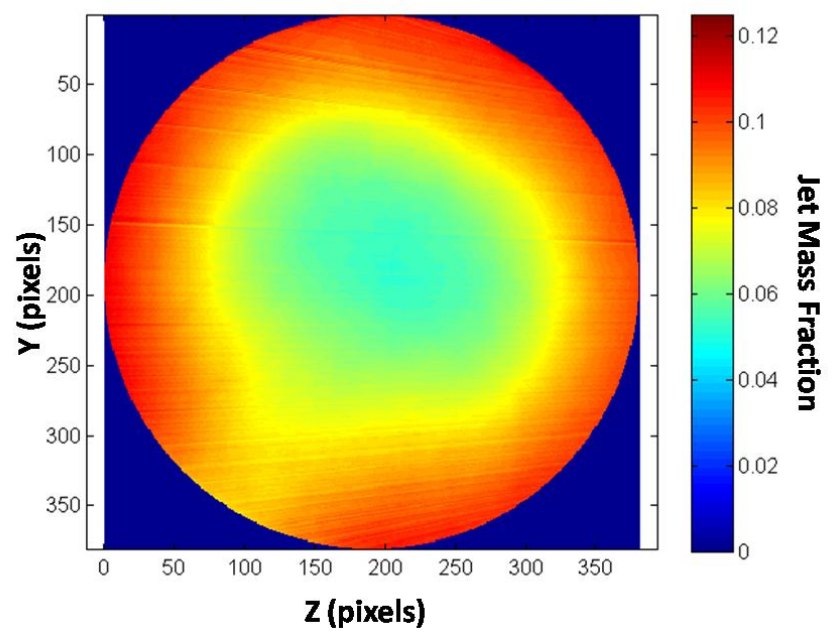

Figure 48: Jet mass fraction for $n=1, B(d / D)=1.5$ 
The two jet trend shown in Fig. 44 has significant differences compared to the single jet data. The mixing performance decreases monotonically for the regime analyzed. Within the given resolution of the $B(d / D)$ data collected, it appears that no local minimum exists in the same region of interest as the single jet. The hypothesized reason for this is two-fold. First, the impaction of two jets of equal and opposite momentum behaves differently than that of a jet impacting the pipe wall. When a single jet impacts a wall, the stream bifurcates and sticks to wall, limiting the interfacial area available to mix with the primary flow. When two jets impact in the center of the pipe, the streams act like an elastic collision and send the jet fluid back towards the wall where each originated. This causes the streams to act more like a free jet, providing more total mixing area than a single jet. The second reason is that by introducing a second blockage, the crossflow is constricted and locally accelerates around the two jets, promoting greater mixing in the two halves of the pipe after the jets have impacted one another.

The data for three jets indicate the presence of a weak local minimum near $B(d / D)=0.87$. However, between approximately $0.75<B(d / D)<1.5$ mixing remains virtually flat despite doubling the jet velocity. This indicates that much of the radial momentum is transformed to axial momentum. By looking at the processed concentration images in this regime (see C-G on summary plot $n=3$, Fig. 56), it is clear that a high concentration center jet stream exists for these levels of $B(d / D)$.

Six jets contain perhaps the most interesting information for all the data sets. A local minimum exist at a $B(d / D)$ lower than any of the other jet numbers producing the lowest unmixedness measured among all the data. This minimum $B(d / D)$ is approximately 0.5 , obtaining an unmixedness about six times lower than one or two jets and four times lower than three jets. However, mixing performance of six jets quickly degrades to a state worse than two or three jets at moderate $B(d / D)$ and even worse than a single jet at high $B(d / D)$. Vranos et al. [24] tested a similar configuration with six slanted holes in a converging-diverging test section. These unmixedness values are plotted against the six jet configuration from the current effort in figure 49. Values of $\mathrm{d} / \mathrm{D}$ for slotted configurations were calculated using the equivalent hydraulic diameter and the diameter of the primary flow at the centerplane of the slots. Note 
that these configurations differ in both test section geometry and axial location of measurement. It is interesting to note that six jets in a constant section mixer achieves a lower unmixedness at low values of $B(d / D)$, yet performs poorly above $B(d / D)=0.87$ despite a greater axial length over which to mix. Six at higher $B(d / D)$ values seem to coalesce at the center of the pipe which reduces the overall interfacial area between jet and crossflow and weakens each individual CVP structure. Six slots as tested by Vranos et al. seem to to suffer from this, most likely due to "channeling the primary flow between each slot like six circumferentially spaced nozzles.

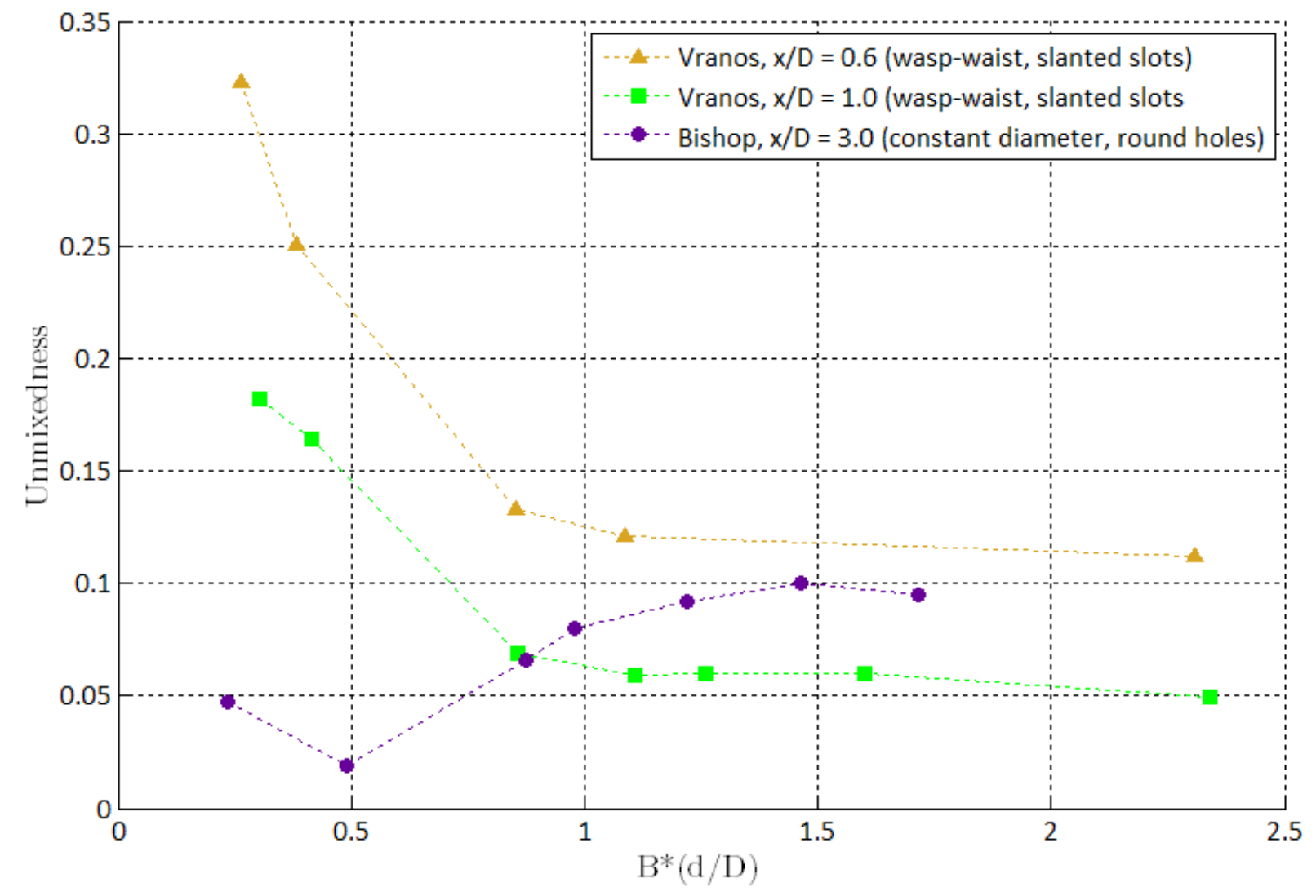

Figure 49: Unmixedness of six jet configurations at various x/D. Data from Vranos et al. [24].

It is also desirable to estimate how local optima for sparse numbers of jets deviate from the scaling law described by the Holdeman relation given by equation 11. By calculating the minimum $B(d / D)$ for the three and six jet curve fits, the momentum flux ratio can be backed out and compared to the value predicted by Holdeman. Figure 50 illustrates these values for three and six jets. For three jets, 
the optimum momentum flux ratio is approximately 17.5 and for six jets it is approximately 8.1 . The six jet value is very nearly equal to the value predicted by Holdeman's scaling law $(\sim 11.4)$ within the limits described by variation in his scaling parameter C. It is worth noting that Holdeman's studies focused further upstream $(x / D=0.5$ to 1.0$)$ but the trends seem to hold true for $x / D=3$. Due to the difference in mixing mechanics described above data for one and two jets are not included in this plot.

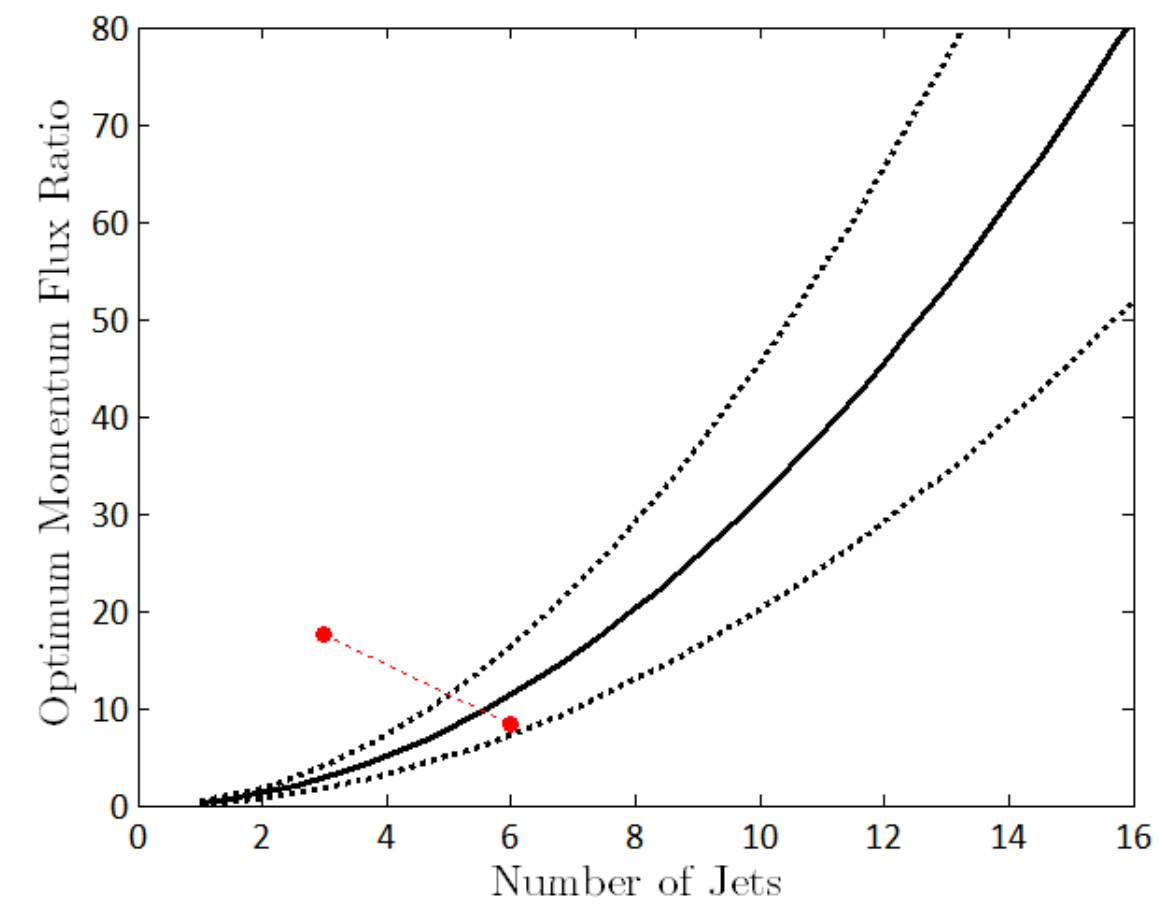

Figure 50: Calculated optimum momentum flux ratio based upon relative unmixedness. Spread of Holdeman data is described by dotted lines.

\subsection{Mixing Performance as a Function of Number of Jets}

Figure 51 shows a composite graph illustrating the effect of number of jets on mixing with the non-dimensional scaling parameter $B(d / D)$ held roughly constant. The target values ranged from 0.25 1.75 in increments of 0.25 . The actual values of $B(d / D)$ vary nominally within three percent of the stated value, due to the available precision of setting the jet and main flow rates from case to case. As such, the results of figure 51 are intended for qualitative use only to identify trends in mixing by changing the 
number of jets at a relatively constant $B(d / D)$. The data indicate that for low values of $B(d / D)$ between 0.25-0.5, a designer can expect better mixing performance by moving to a higher number of jets. This is illustrated by the red and green curves respectively. At moderate $B(d / D)$ between 0.75 and 1.0 , an optimum number of jets exists below six (see blue and cyan curves). Because four and five jets were not measured, it cannot be determined at this time if either of those conditions would result in more optimal mixing than three jets. Finally, at high $B(d / D)$, it appears that the best mixing performance is obtained by using two jets, as indicated by the magenta, gold, and black curves.

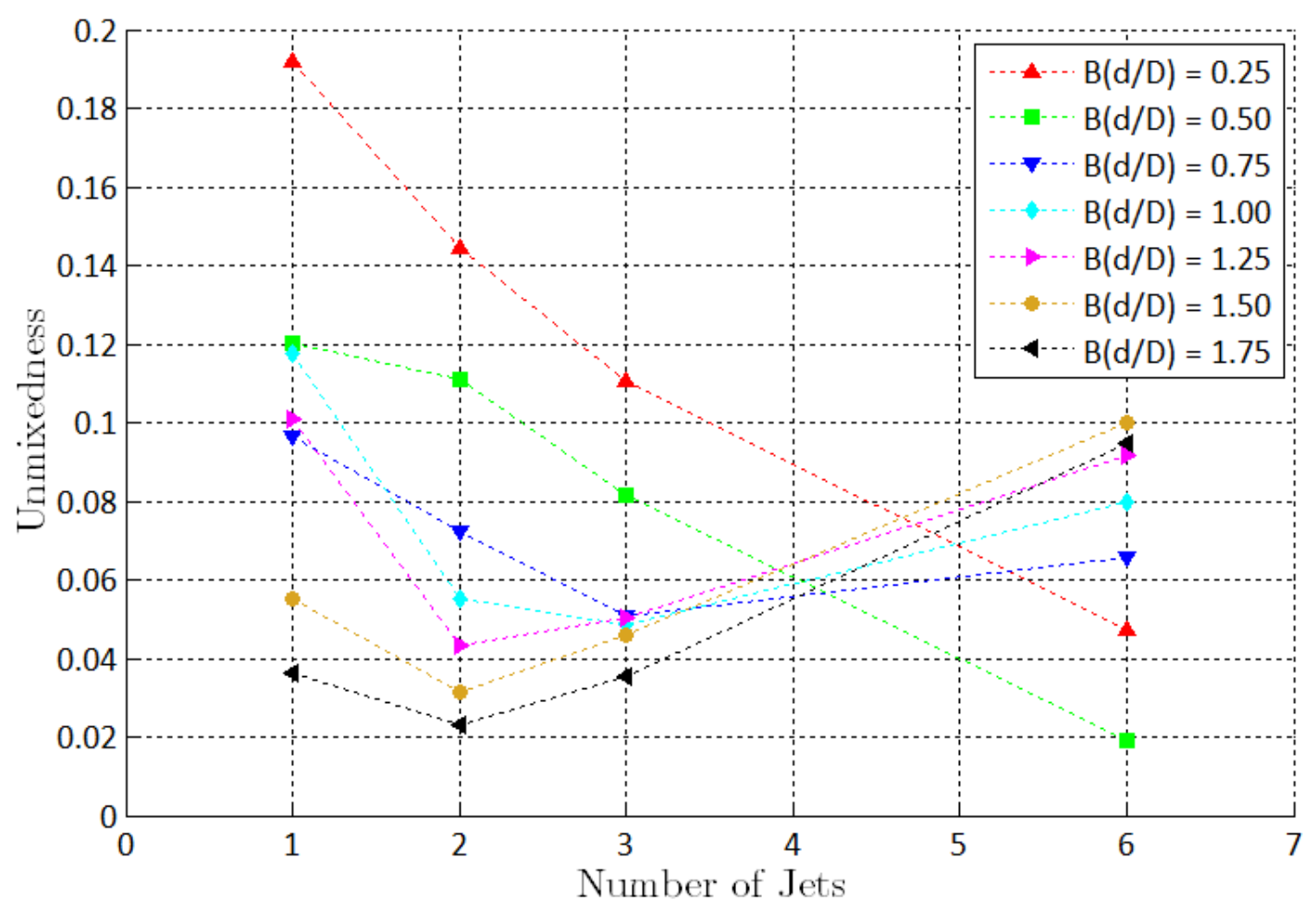

Figure 51: Unmixedness as a function of the number of jets.

\subsection{Mixing Performance as a Function of Momentum Ratio}

Correlation data from Sroka and Forney [20] provides a comparison which the current data can be evaluated as a function of momentum ratio. Figure 52 illustrates the location-adjusted second moment of concentration, $M(x / D)^{4 / 3}=0.25 \beta^{-1}$, where 0.25 is the best fit correlation coefficient. The original equation factors $x / D$ into the mixedness evaluation $M$, so multiplying by the $4 / 3$ term 
normalizes these measurements for comparison to any axial location. The correlation (valid only for a single jet) provides a reasonable estimate of the second moment for the lower values of $B(d / D)$ at $n=1$. The deviation at higher $B(d / D)$ is expected as the jet begins to approach the wall impaction regime. Sroka and Forney [20] seem to suggest a monotonic mixing improvement with momentum ratio. However, the scatter apparently in their data at momentum ratios near one is likely due to diameter ratio effects. Not surprisingly, the data for higher numbers of jets falls below the single jet correlation to varying levels. It is interesting to note that a momentum ratio of 0.4 seems to be a point at which all curves for multiple jets approach the same level of second moment. A future scientific study might make use of this information to study different geometries and identify and similarities and differences in flow structures that somehow result in the same value for second moment of concentration (although this may also be coincidental).

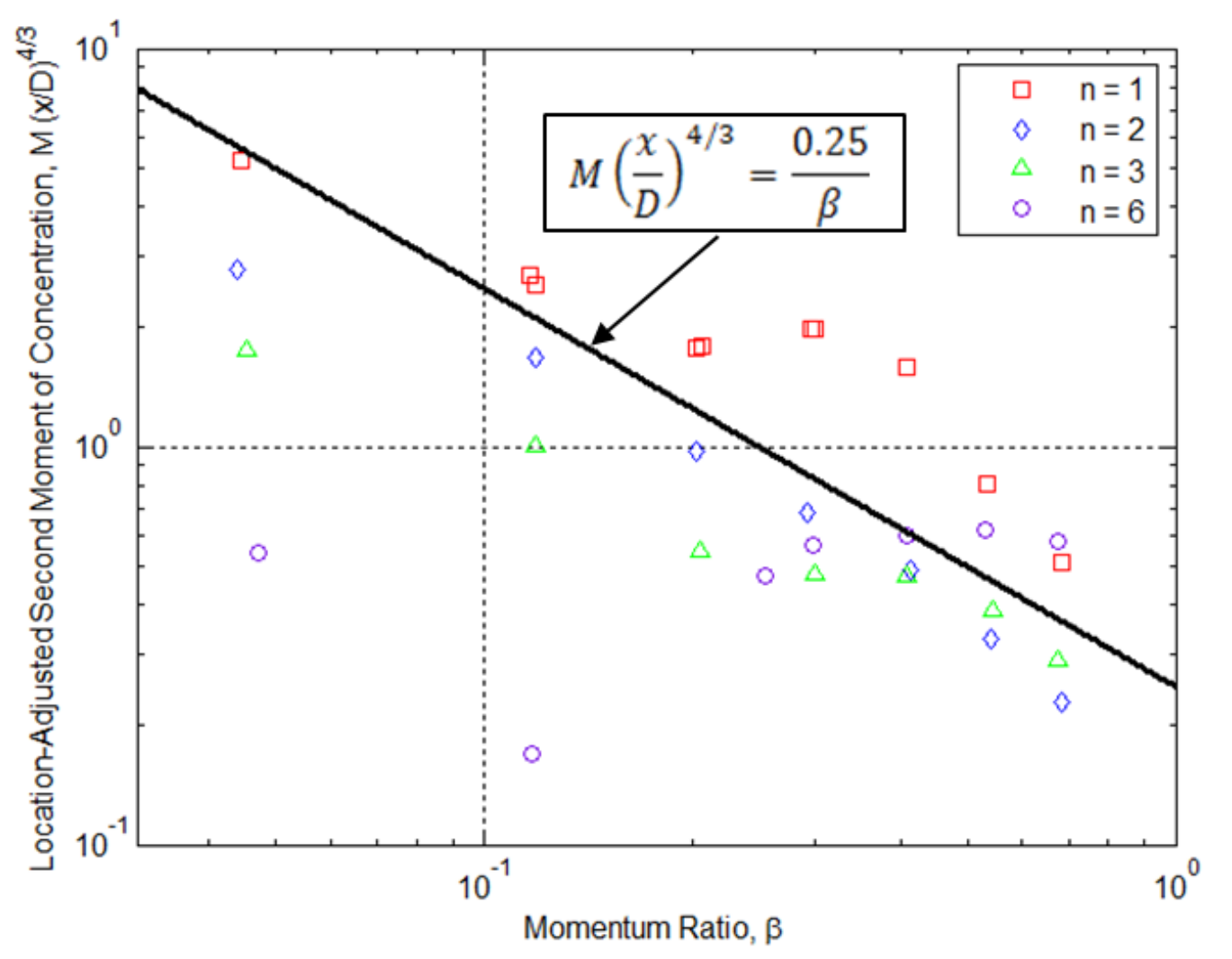

Figure 52: Location-adjusted second moment of concentration versus momentum ratio.

Correlation from Sroka and Forney [20]. 
Another useful way of visualizing mixing performance is to plot unmixedness versus total momentum ratio, the ratio of how much total momentum is being imparted through the jets versus the primary flow. Figure 53 illustrates these data. Within limits of the design space, the data indicate the most effective way to achieve effective mixing depends on how much total momentum is available to impart to the jet fluid. With sparse numbers of jets, the data suggest that raising the total momentum ratio as high as possible will continually improve mixing. The six jet configuration seems to be a very effective compromise, provided the designer selects a total momentum ratio below unity.

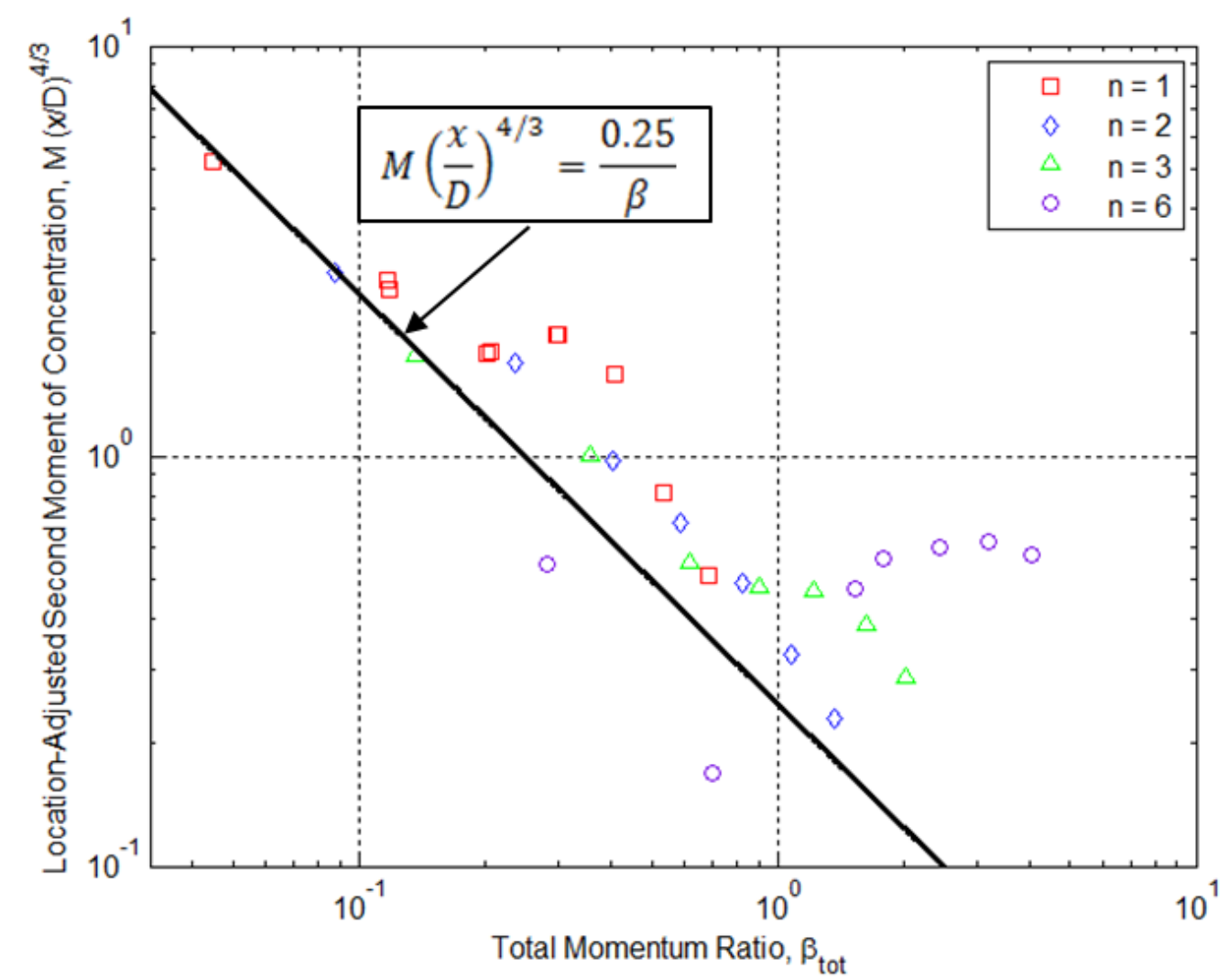

Figure 53: Location-adjusted second moment of concentration versus total momentum ratio. Correlation from Sroka and Forney [20]. 


\subsection{Summary Plots}

The next several pages contain summary plots with calculated mass fractions at each jet number, along with their individual plots $B(d / D)$ versus unmixedness and momentum ratio versus $M(x / D)^{4 / 3}$. The images are labeled by their target mean mass fraction based on volumetric flow rate ratio. These values approximately match the calculated mean value for each image. 

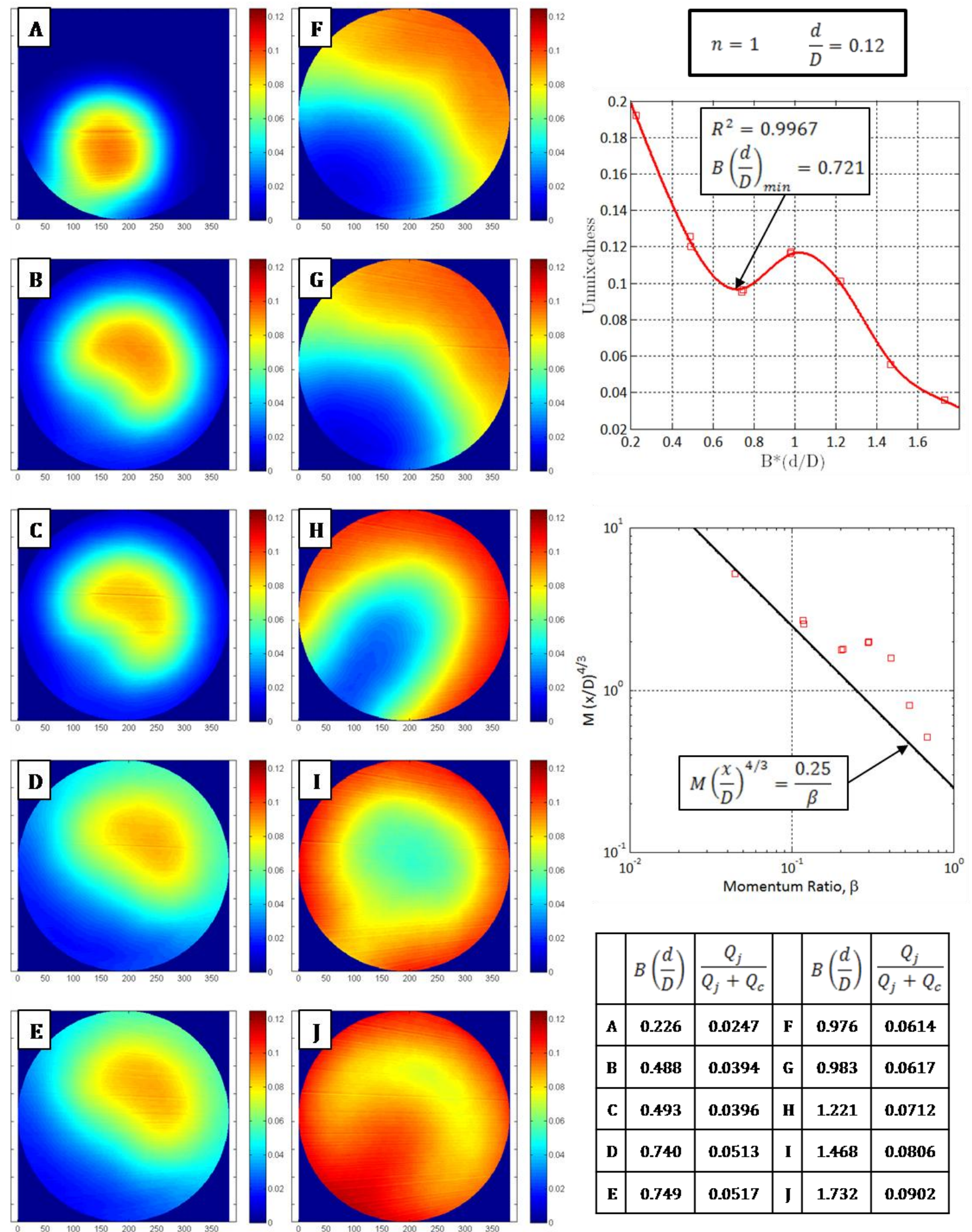

\begin{tabular}{|c|c|c|c|c|c|}
\hline & $B\left(\frac{d}{D}\right)$ & $\frac{Q_{j}}{Q_{j}+Q_{c}}$ & & $B\left(\frac{d}{D}\right)$ & $\frac{Q_{j}}{Q_{j}+Q_{c}}$ \\
\hline A & 0.226 & $\mathbf{0 . 0 2 4 7}$ & F & 0.976 & $\mathbf{0 . 0 6 1 4}$ \\
\hline B & 0.488 & $\mathbf{0 . 0 3 9 4}$ & G & 0.983 & $\mathbf{0 . 0 6 1 7}$ \\
\hline C & $\mathbf{0 . 4 9 3}$ & $\mathbf{0 . 0 3 9 6}$ & H & 1.221 & $\mathbf{0 . 0 7 1 2}$ \\
\hline D & $\mathbf{0 . 7 4 0}$ & $\mathbf{0 . 0 5 1 3}$ & I & 1.468 & $\mathbf{0 . 0 8 0 6}$ \\
\hline E & $\mathbf{0 . 7 4 9}$ & $\mathbf{0 . 0 5 1 7}$ & J & 1.732 & $\mathbf{0 . 0 9 0 2}$ \\
\hline
\end{tabular}

Figure 54: Summary plot for single jet configuration. 

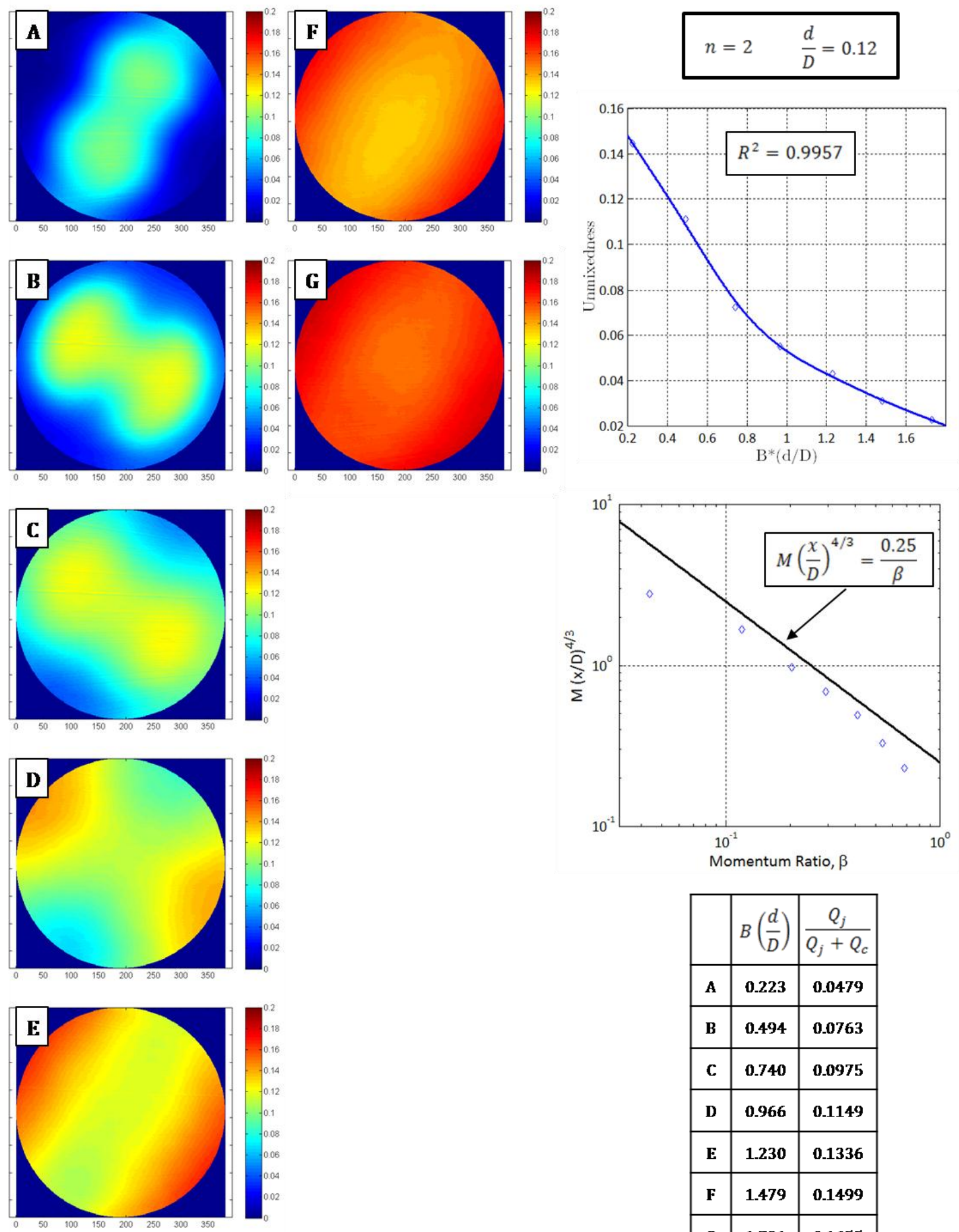

\begin{tabular}{|c|c|c|}
\hline & $B\left(\frac{d}{D}\right)$ & $\frac{Q_{j}}{Q_{j}+Q_{c}}$ \\
\hline A & 0.223 & 0.0479 \\
\hline B & 0.494 & 0.0763 \\
\hline C & 0.740 & 0.0975 \\
\hline D & 0.966 & 0.1149 \\
\hline E & 1.230 & 0.1336 \\
\hline F & 1.479 & 0.1499 \\
\hline G & 1.731 & 0.1655 \\
\hline
\end{tabular}

Figure 55: Summary plot for two jet configuration. 

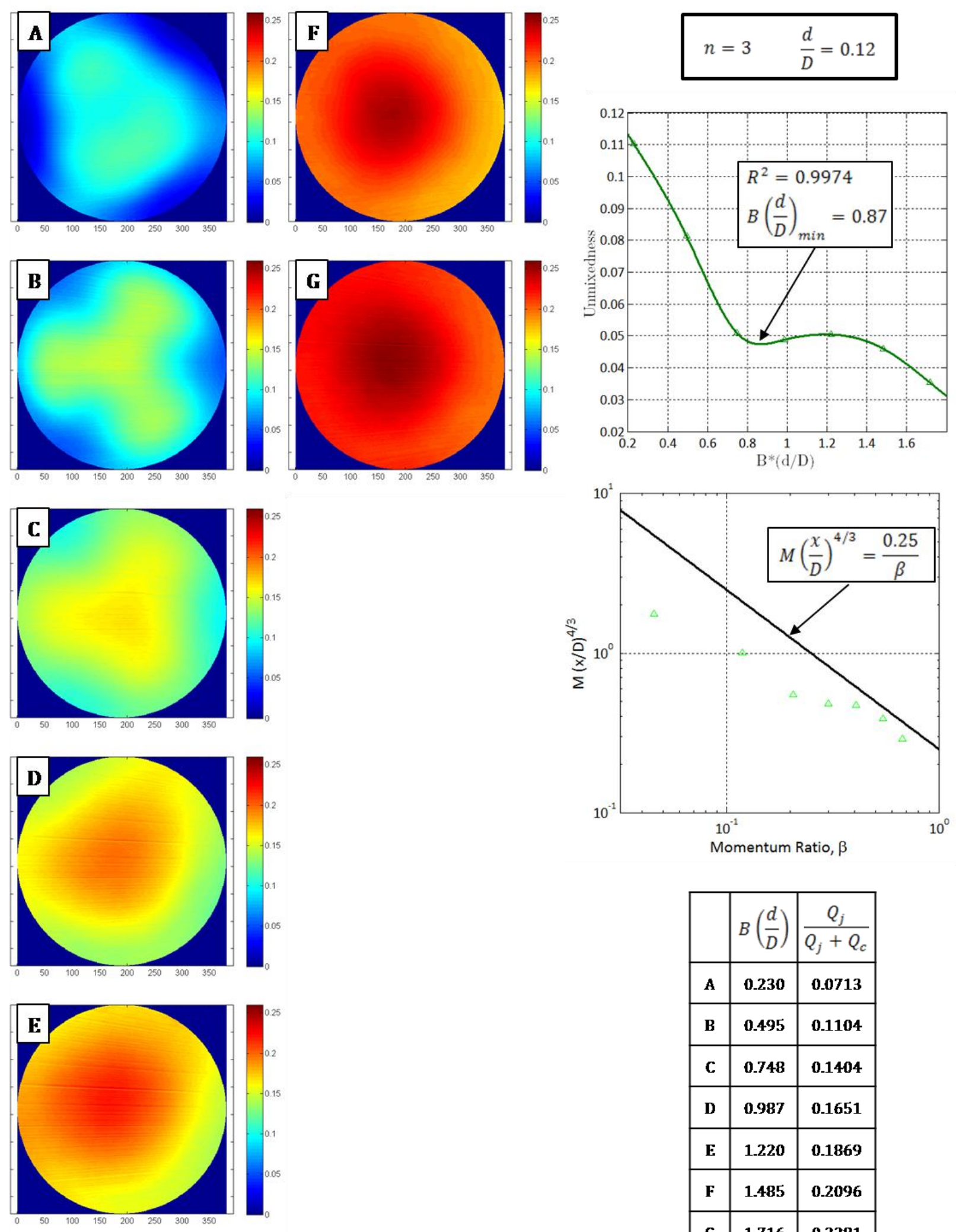

\begin{tabular}{|c|c|c|}
\hline & $B\left(\frac{d}{D}\right)$ & $\frac{Q_{j}}{Q_{j}+Q_{c}}$ \\
\hline A & 0.230 & 0.0713 \\
\hline B & 0.495 & 0.1104 \\
\hline C & 0.748 & 0.1404 \\
\hline D & 0.987 & 0.1651 \\
\hline E & 1.220 & 0.1869 \\
\hline F & 1.485 & 0.2096 \\
\hline G & 1.716 & 0.2281 \\
\hline
\end{tabular}

Figure 56: Summary plot for three jet configuration. 

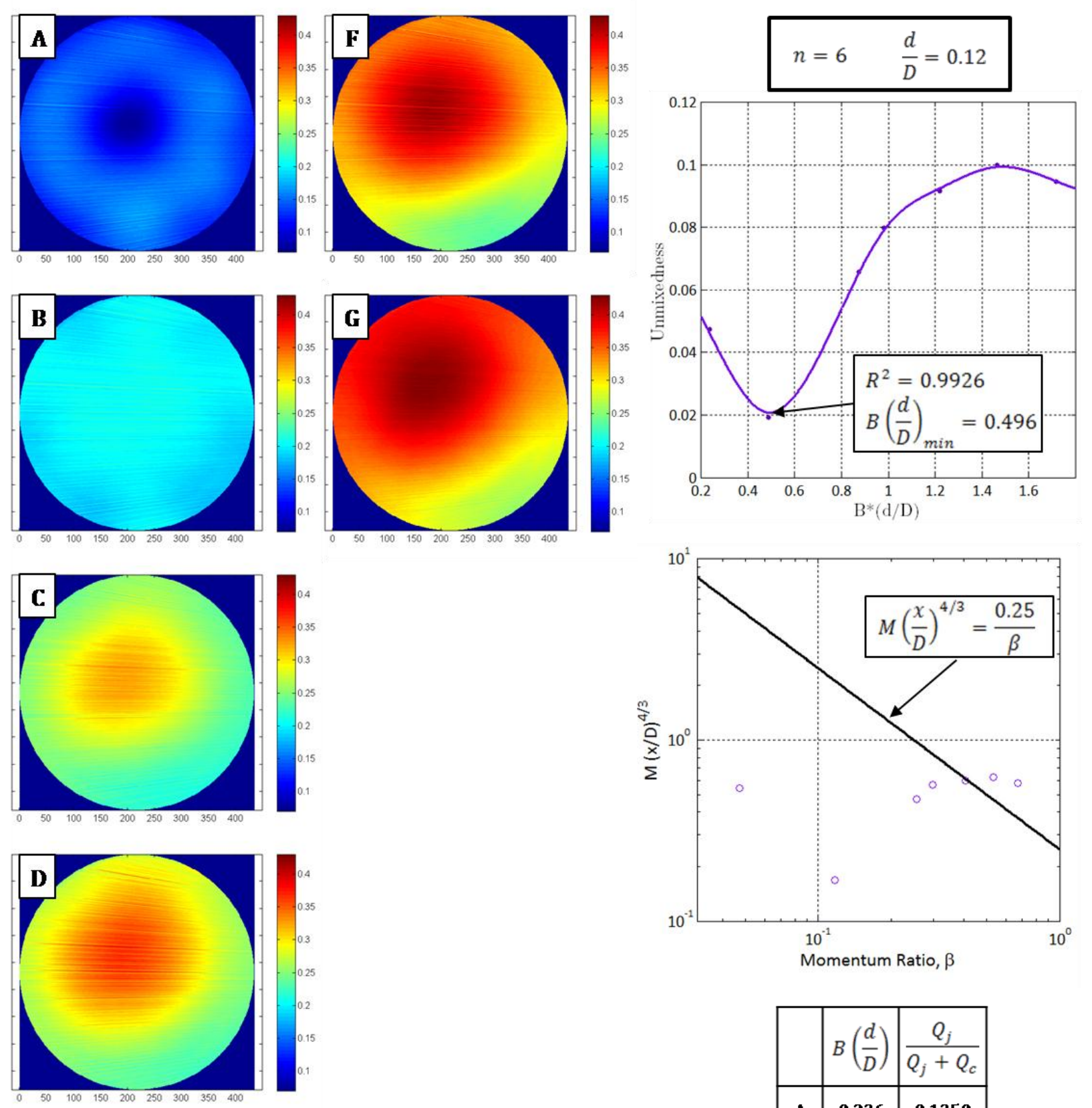

\begin{tabular}{|c|c|c|}
\hline & $B\left(\frac{d}{D}\right)$ & $\frac{Q_{j}}{Q_{j}+Q_{c}}$ \\
\hline A & $\mathbf{0 . 2 3 6}$ & $\mathbf{0 . 1 3 5 0}$ \\
\hline B & 0.489 & 0.1976 \\
\hline C & 0.875 & 0.2667 \\
\hline D & 0.980 & 0.2824 \\
\hline E & 1.219 & 0.3148 \\
\hline F & 1.464 & 0.3443 \\
\hline G & 1.717 & 0.3715 \\
\hline
\end{tabular}

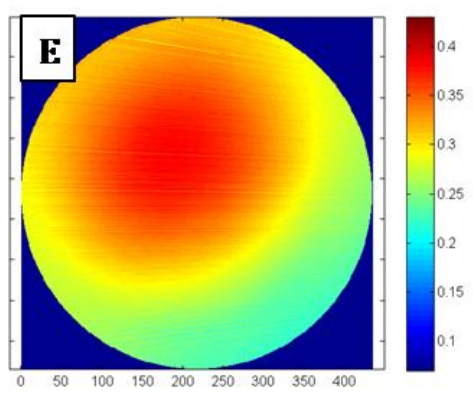

Figure 57: Summary plot for six jet configuration. 


\subsection{Summary and Conclusions}

The mixing performance of multiple transverse jets has been evaluated experimentally. Basic findings are consistent with results presented in literature for single jet mixing behavior. Mixing performance has been compared to literature for the single jet case and the Holdeman parameter has been re-evaluated for effectiveness at low jet numbers. A single jet in a confined crossflow was found to have a local minimum at $B(d / D)=0.721$. Results for two jets indicate monotonically decreasing unmixedness for the range of conditions tested, with no local optimum apparent. Data for three jets indicate a local optimum at $B(d / D)=0.87$ and relatively flat range of mixing performance in the range of $0.75<$ $B(d / D)<1.25$. Six jets indicate a minimum unmixedness near $B(d / D)=0.5$, but exhibited poorer mixing performance than all other configurations at the highest values of $B(d / D)$ tested. The most optimum configuration tested was six jets at $B(d / D)=0.5$, resulting in an unmixedness of 0.0192 . This value was $76 \%$ lower than the next lowest configuration (three jets) at the same $B(d / D)$. Total momentum was found to collapse the data well, as configurations more closely matched a historical correlation for second moment of a single confined jet more closely.

Recommendations for future work include completing the current study to include more diameter ratios to test the robustness of the scaling law $B(d / D)$ derived by Forliti [36]. This includes extreme diameter ratios down to the 0.04 range (as tested by Maruyama et al. [13] with 1 and 2 jets) and up to the 0.25 range (tested by Hatch et al. [25] with 8 jets). PLIF served as a very useful measurement system for extracting scalar mixing data. The calibration process was able to successfully mitigate the effects of moving cameras, fluctuations in the laser light sheet from day-to-day and base concentration differences from different batches of pre-mixed jet fluid. However, using PLIF necessitates scaling down the flow system so as to minimize the waste fluid generated per unit time during testing. This proved to be a logistical difficulty, but once the system became more familiar to all users/operators, full ranges of data points could be taken in a matter of days. 


\section{Bibliography}

1. Pratt and Whitney Rocketdyne, "Space Launch Solutions." Corporate website. http://www.pw.utc.com/products/pwr/propulsion solutions/space launch.asp

2. Space Exploration Technologies, "Falcon 9 Overview." Corporate website. http://www.spacex.com/falcon9.php

3. Fric, T. and Roshko, A., "Vortical Structure in the Wake of a Transverse Jet," Journal of Fluid Mechanics, Vol. 279, 1994, pp. 1-47.

4. Hasselbrink, E.F. and Mungal, M.G., "Transverse jets and jet flames. Part 1. Scaling laws for strong transverse jets," Journal of Fluid Mechanics, Vol. 443, 2001, pp. 1-25.

5. Kelso, R.M., Lim, T.T. and Perry, A.E., "An Experimental Study of Round Jets in Cross-Flow," Journal of Fluid Mechanics, Vol. 306, 1996, pp. 111-144.

6. Lim, T., New, T. and Luo, S., "On the Development of Large-Scale Structures of a Jet Normal to a Cross Flow," Physics of Fluids, Vol. 13, No. 3, 2001, pp. 770-775.

7. Smith, S. and Mungal, M., "Mixing, structure and scaling of the jet-in-crossflow," Journal of Fluid Mechanics, Vol. 357, 1998, pp. 83-122.

8. Kelso, R. and Smits, A., "Horseshoe Vortex Systems Resulting from the Interaction between a Laminar Boundary Layer and a Transverse Jet,” Physics of Fluids, Vol. 7, No. 1, 1995, pp. 153158.

9. Keffer, J.F. and Baines, W.D., "The round turbulent jet in a cross-wind," Journal of Fluid Mechanics, Vol 15, 1963, pp. 481-496.

10. Pratte, B.D. and Baines, W.D., "Profiles of the round turbulent jet in cross flow," Journal of Hydronautics Div. ASCE, Vol. 92, 1967, pp. 53-64.

11. Wu, J., "Near-Field Trajectory of Turbulent Jets Discharged at Various Inclinations into a Uniform Crossflow,” AIAA Journal, Vol. 11, No. 11, 1973, pp. 1579-1581. 
12. Kamotani, Y. and Greber, I., "Experiments on a turbulent jet in a cross flow." AIAA Journal, Vol. 10, 1972, pp. 1425-1429.

13. Maruyama, T., Mizushina, T. and Hayashiguchi, S., "Optimum conditions for jet mixing in turbulent pipe flow," International Chemical Engineering, Vol. 23, No. 4, 1983, pp. 707-716.

14. Muppidi, S. and Mahesh, K., "Study of Trajectories of Jets in Crossflow Using Direct Numerical Simulations," Journal of Fluid Mechanics, Vol. 530, 2005, pp. 81-100.

15. Mashayek, A., Jafari, A. and Ashgriz, N., "Improved Model for the Penetration of Liquid Jets in Subsonic Crossflows,” AIAA Journal, Vol. 46, No. 11, 2008, pp. 2674-2686.

16. Kamotani, Y. and Greber, I., "Experiments on Confined Turbulent Jets in Cross Flow," NASA CR-2392, March 1974.

17. Margason, R.J., "Fifty Years of Jet n Cross Flow Research," In AGARD, Computational and Experimental Assessment of Jets in Cross Flow, SEE N94-28003 07-34, 1993, pp. 1-41.

18. Broadwell, J.E. and Breidenthal, R.E., "Structure and mixing of a transverse jet in incompressible flow," Journal of Fluid Mechanics, Vol. 148, 1984, pp. 405-412.

19. New, T., Lim, T. and Luo, S., "Effects of Jet Velocity Profiles on a Round Jet in Cross-Flow," Experiments in Fluids, Vol. 40, No. 6, 2006, pp. 859-875.

20. Sroka, L.M. and Forney, L.J., "Fluid Mixing with a Pipeline Tee: Theory and Experiment," AIChE Journal, Vol. 35, No. 3, 1989, pp. 406-414.

21. Pan, G. and Meng, H., "Experimental Study of Turbulent Mixing in a Tee Mixer Using PIV and PLIF," AIChE Journal, Vol. 47, No. 12, 2001, pp. 2653-2665.

22. Holdeman, J.D., Walker, R.E. and Kors, D.L., "Mixing of Multiple Dilution Jets with a Hot Primary Airstream for Gas Turbine Combustors," NASA TM X-71426, Presented at the $9^{\text {th }}$ Propulsion Conference (AIAA/SAE), Las Vegas, NV, 5-7 Nov. 1973.

23. Holdeman, J.D., "Mixing of Multiple Jets with a Confined Subsonic Crossflow," Progress in Energy and Combustion Science, Vol. 19, 1993, pp. 31-70. 
24. Vranos, A., Liscinsky, D.S., True, B., and Holdeman, J.D., "Experimental Study of Cross-Stream Mixing in a Cylindrical Duct," NASA TM-105180, Presented at the $27^{\text {th }}$ Joint Propulsion Conference (AIAA, SAE, ASME, ASEE), Sacramento, CA, 24-27 June 1991.

25. Hatch, M.S., Sowa, W.A., Samuelsen, G.S. and Holdeman, J.D., "Jet Mixing Into a Heated Cross Flow in a Cylindrical Duct: Influence of Geometry and Flow Variations," NASA TM-105390, Presented at the $30^{\text {th }}$ Aerospace Sciences Meeting and Exhibit (AIAA), Reno, NV, 6-9 Jan. 1992.

26. Holdeman, J.D., Liscinsky D.S., Oechsle, V.L., Samuelsen, G.S. and Smith, C.E., "Mixing of Multiple Jets with a Confined Subsonic Crossflow: Part 1-Cylindrical Duct," Journal of Engineering for Gas Turbines and Power, Vol. 119, 1997, pp. 852-862.

27. Koochesfahani, M.M. and Dimotakis, P.E., "Laser Induced Fluorescence Measurements of Mixed Fluid Concentration in a Liquid Plane Shear Layer," AIAA Journal, Vol. 23, No. 11, 1985, pp. $1700-1707$.

28. Koochesfahani, M.M. and Dimotakis, P.E., "Mixing and chemical reactions in a turbulent liquid mixing layer," Journal of Fluid Mechanics, Vol. 170, 1986, pp. 83-112.

29. Walker, D.A., "A fluorescence technique for measurement of concentration in mixing liquids," Journal of Physics E: Scientific Instruments, Vol. 20, 1987, pp. 217-224.

30. Koochesfahani, M.M., Dimotakis, P.E. and Broadwell, J.E., “A 'Flip’ Experiment in a Chemically Reacting Turbulent Mixing Layer,” AIAA Journal, Vol. 23, No. 8, 1985, pp. 11911194.

31. Koochesfahani, M.M. and MacKinnon, C.G., "Influence of forcing on the composition of mixed fluid in a two-stream shear layer," Physics of Fluids A, Vol. 3, No. 5, 1991, pp 1135-1142.

32. van Cruyningen, I., Lozano, A. and Hanson, R.K., "Quantitative imaging of concentration by planar-laser-induced fluorescence," Experiments in Fluids, Vol. 10, 1990, pp. 41-49.

33. Shan, J.W. and Dimotakis, P.E., "Turbulent mixing in transverse jets," GALCIT Report CalechGalcitFM:2001.006, 2001. 
34. Karasso, P.S. and Mungal, M.G., "PLIF measurements in aqueous flows using the Nd:YAG laser,” Experiments in Fluids, Vol. 23, 1997, pp. 382-387.

35. Shan, J.W., Lang, D.B. and Dimotakis, P.E., "Scalar concentration measurements in liquid-phaseflows with pulsed lasers," Experiments in Fluids, Vol. 36, 2004, pp. 268-273.

36. Forliti, D.J., "Trajectory and Mixing Scaling Laws for Confined and Unconfined Transverse Jets," 42 ${ }^{\text {nd }}$ AIAA Fluid Dynamics Conference and Exhibit, 2012.

37. Ricou, F., and Spalding, D., "Measurements of entrainment by axisymmetrical turbulent jets," Journal of Fluid Mechanics Vol. 11, 1961, pp. 21-32.

38. Van Doorne, C.H.W. and Westerweel, J., "Measurement of laminar, transitional and turbulent pipe flow using Stereoscopic-PIV," Experiments in Fluids, Vol. 32, No. 2, 2007, pp. 259-279.

39. Den Toonder, J.M.J and Nieuwstadt, F.T.M., "Reynolds number effects in a turbulent pipe flow for low to moderate Re," Physics of Fluids, Vol. 9, No. 11, 1997 pp. 3398-3409.

40. Ajayi, K.T., Papadopulos, G. and Durst, F., "Influence of upstream development on the losses incurred by flow past an axisymmetric sudden contraction," AIAA 1998-0764, 36 ${ }^{\text {th }}$ Aerospace Sciences Meeting and Exhibit, Reno, NV, 1998. 


\section{Appendices}

\section{Appendix A: Laser Doppler Velocimetry}

Verification of the inflow profiles was necessary to ensure that the inflow condition was fullydeveloped pipe flow. This includes any misalignment of piping coming into the jet/crossflow intersection. The method chosen to document these profiles was Laser Doppler Velocimetry (LDV) which works on the principle of interference. Two laser beams are oriented at a converging angle, such that they create a volume in which their signals create alternating bands of constructive and destructive interference. As particles pass through these bright and dark fringes, they scatter light which is collected by a photodetector. A single light burst is analyzed to acquire a single velocity measurement that represents a single particle traveling through the probe volume. Ensembles of these instantaneous measurements are used to calculate time-averaged statistics for fluid velocity. Figure A1 illustrates the geometry of the interference region.

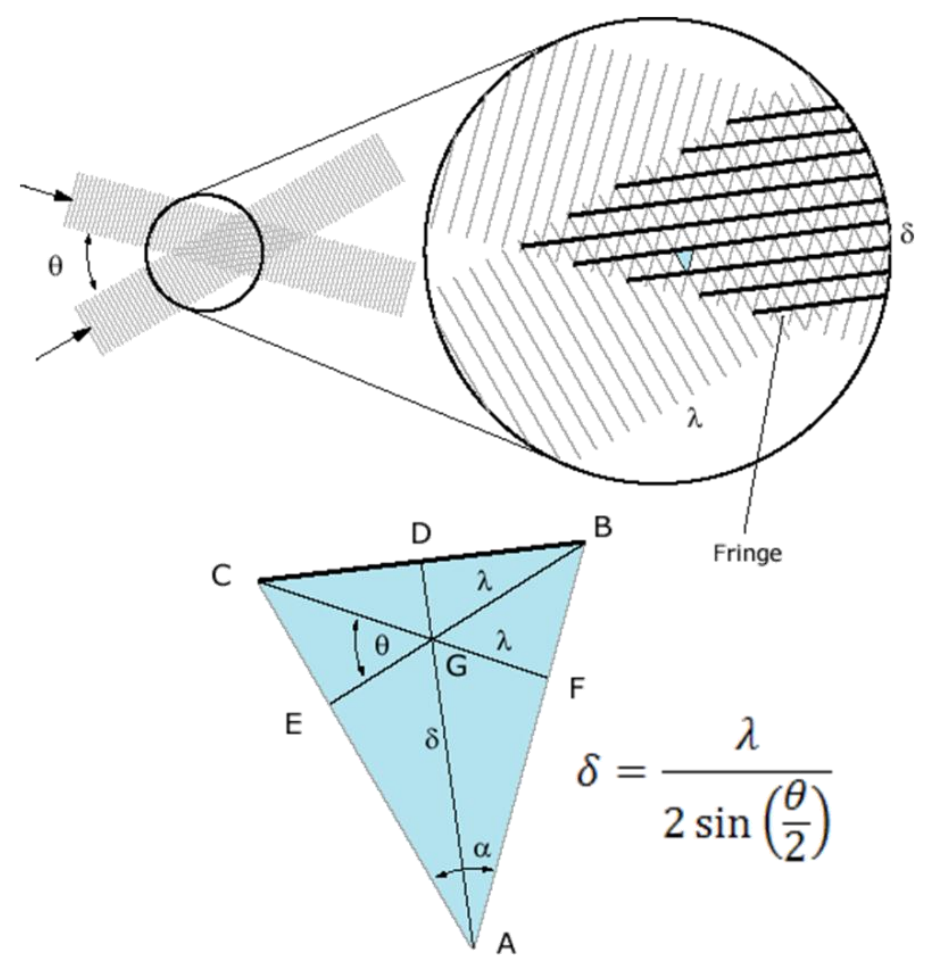

Figure A1: Interference pattern of an LDV setup. 
The LDV system used was a miniLDV by Measurement Sciences Inc. The system uses a $60 \mathrm{~mW}$ diode laser with all optics and a photodetector housed in a single unit, linked to the laser and signal processor via fiber optic cable. It is capable of measuring between $1 \mathrm{~mm} / \mathrm{s}$ to $300 \mathrm{~m} / \mathrm{s}$ at $99.7 \%$ accuracy. Output files report average velocity and turbulent fluctuations. Figures A2, A3 and A4 detail normalized streamwise velocity for three flow rates: 1.61 GPM, 2.34 GPM and 8.30 GPM. Data from van Doorne and Westerweel [38] and den Toonder and Nieuwstadt [39] at similar Reynolds numbers is plotted for comparison.

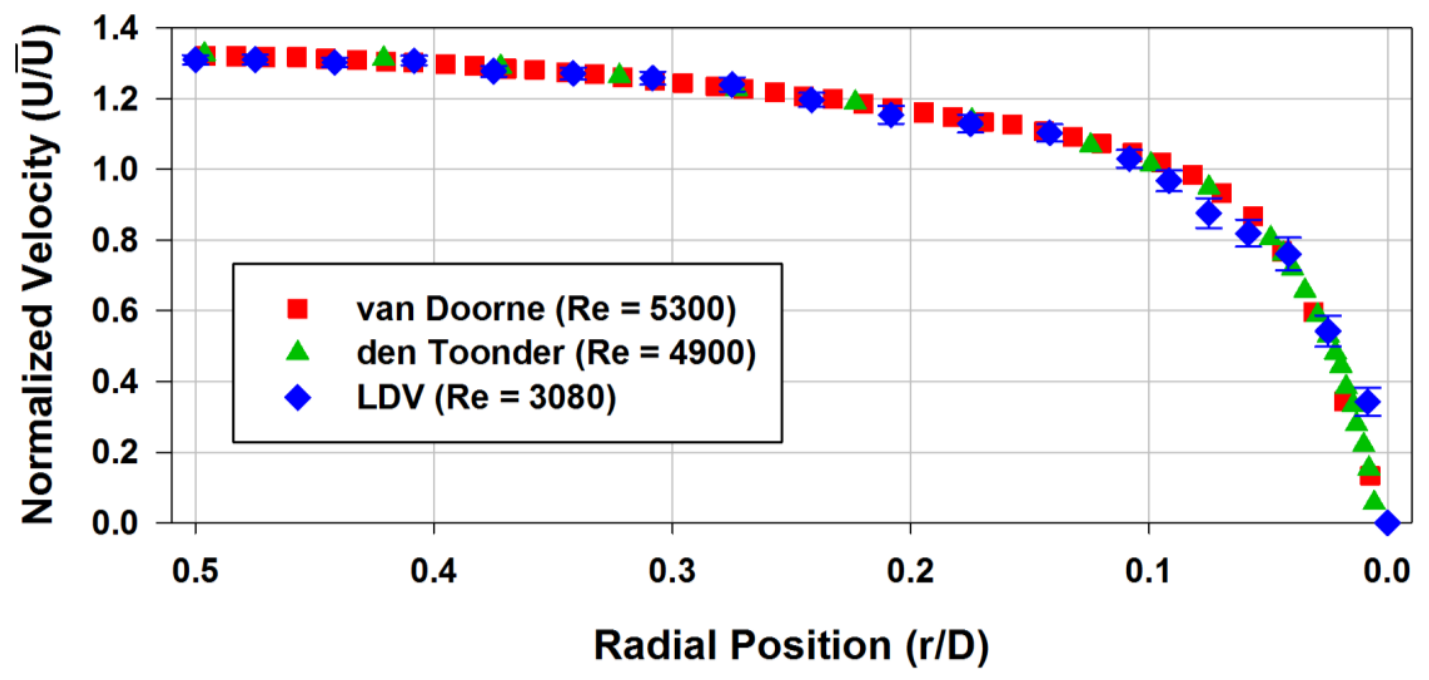

Figure A2: Normalized velocity profile for $Q=1.61$ GPM. Comparison data from van Doorne and Westerweel [38] and den Toonder and Nieuwstadt [39].

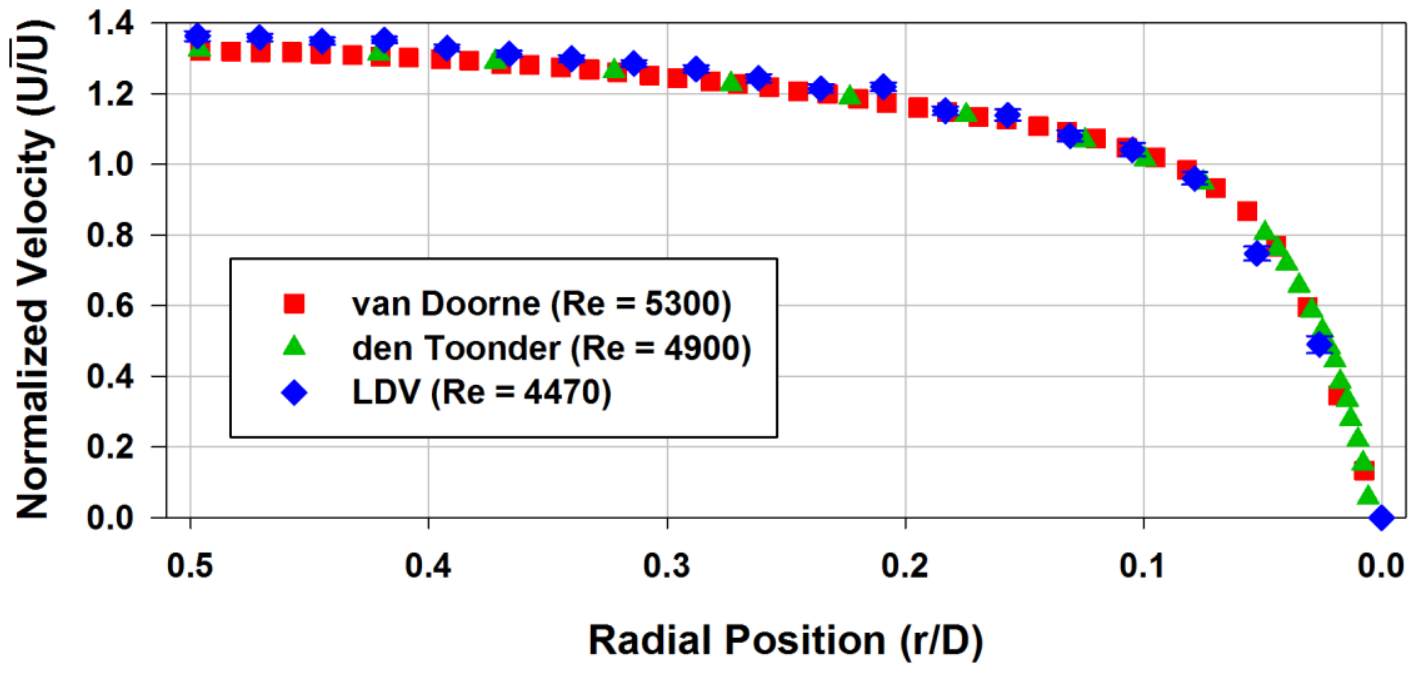

Figure A3: Normalized velocity profile for $Q=2.34$ GPM.

Comparison data from van Doorne and Westerweel [38] and den Toonder and Nieuwstadt [39]. 


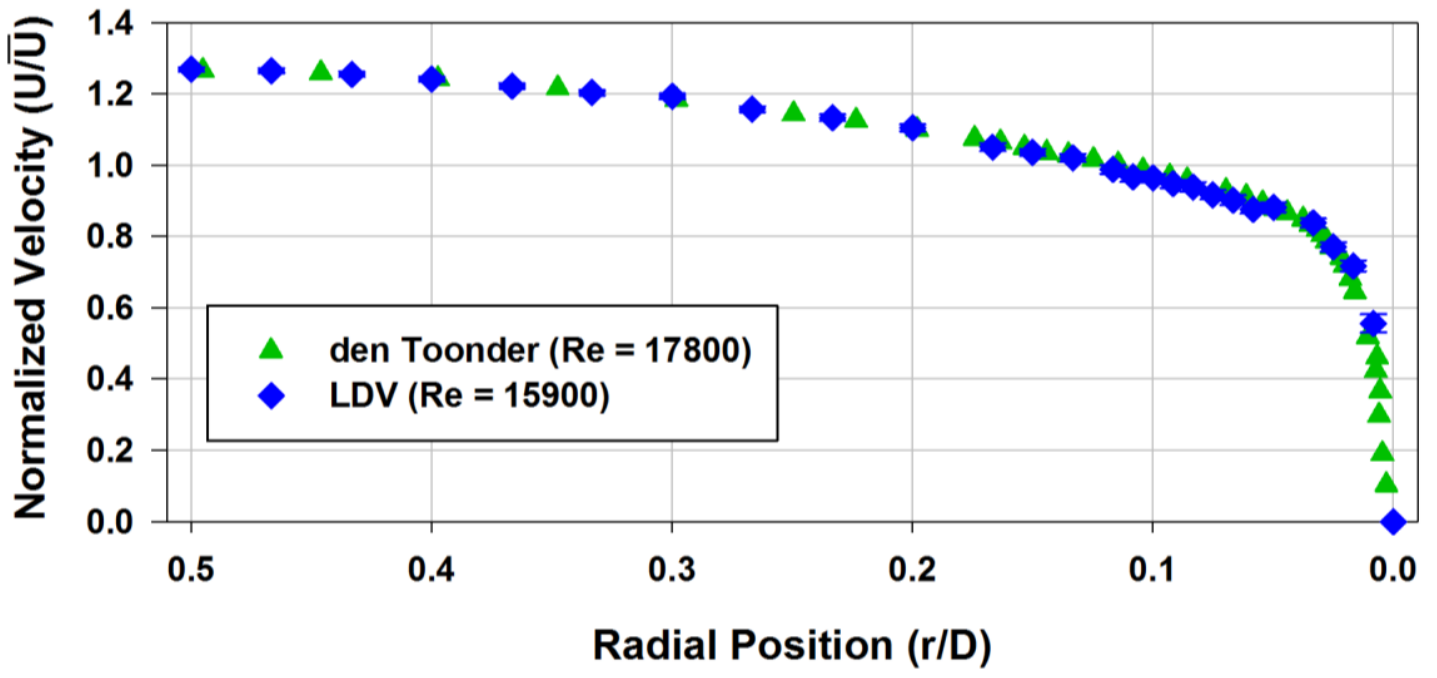

Figure A4: Normalized velocity profile for $Q=8.30$ GPM. Comparison data from den Toonder and Nieuwstadt [39].

The jet inflow profiles were estimated to be turbulent for all injector diameters based on the Reynolds number and geometry. A study by Ajayi, Papadopoulos and Durst [40] concluded that flow past a sudden axisymmetric contraction will re-establish fully-developed flow in approximately 10 diameters past the contraction. Figure A5 illustrates the normalization of the mean velocity as a function of axial distance downstream of a sudden contraction for three different Reynolds numbers. For this reason, the injectors were designed to have L/D values of 20 and greater and pipe Reynolds numbers between 6,000 and 10,000. Turbulent pipe flow for the jets was assumed for all test cases.

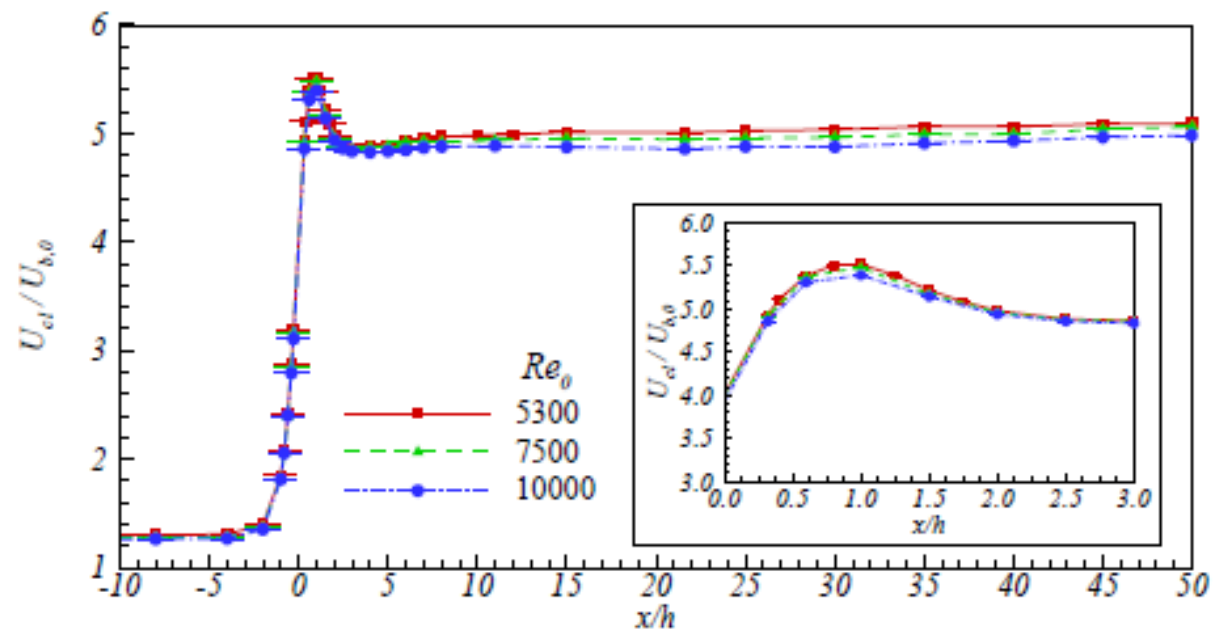

Figure A5: Velocity normalization at a sudden axial contraction. Ajayi, Papadopoulos and Durst [40] 


\section{Appendix B: Derivation of Expected Holdeman Scaling Deviation}

Consider two cases of a round jet issuing into a confined crossflow. The first has a diameter $d_{1}$ and velocity $v_{1}$. The second has a diameter $d_{2}$ and velocity $v_{2}$. Each jet issues into a crossflow with duct diameter $D$ and a velocity $v_{c}$. Each jet has the same density $\rho_{j}$. Figure B1 illustrates the respective cases axially.
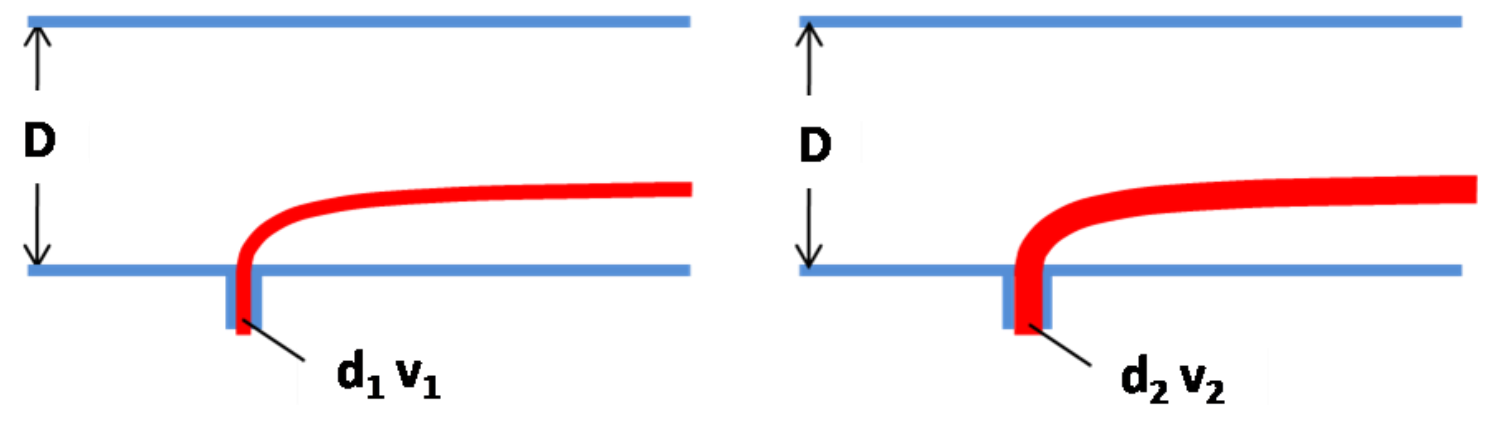

Figure B1: Comparative cases for jet injection.

In order to target a certain bulk mixing, it is desirable to predict the depth of penetration the jet achieves before aligning with the crossflow. An argument may be made that if the momentum ratio of each jet is equal, they will penetrate equally deeply into the crossflow. The definition of the momentum ratio is

$$
\beta=\frac{\rho_{j} v_{j}^{2}\left(\frac{\pi}{4} d^{2}\right)}{\rho_{c} v_{c}^{2}(d D)}
$$

where the area term for the crossflow is representative of the blockage area caused by the jet. Equating the momentum ratios for the two proposed cases yields

$$
\frac{\rho_{j} v_{1}^{2}\left(\frac{\pi}{4} d_{1}^{2}\right)}{\rho_{c} v_{c}^{2}\left(d_{1} D\right)}=\frac{\rho_{j} v_{2}^{2}\left(\frac{\pi}{4} d_{2}^{2}\right)}{\rho_{c} v_{c}^{2}\left(d_{2} D\right)}
$$


We may substitute the momentum flux ratio as $J=\rho_{j} v_{j}^{2} / \rho_{c} v_{c}{ }^{2}$ and cancel constant terms to yield

$$
J_{1}\left(\frac{d_{1}}{D}\right)=J_{2}\left(\frac{d_{2}}{D}\right)
$$

Numerous studies of jet trajectory (list citations) have used the product $r d$ as a scaling metric for strong jets, where $r$ is the blowing ratio (equivalent to the square root of the momentum flux ratio). The spreading of the jet has also been shown to be proportional to $r d$ for numerous cases (references [8] [9] [14] [15]). Consider two jets spaced a distance $S$ apart in a crossflow. When the jet spreads to half the spacing distance, the jets will begin to touch each other and the CVPs will interact to promote better mixing than either would alone. Assume this distance is equivalent to $r d$. If $r d<S$ then the jets will not interact and mixing performance will be reduced. If $r d>S$ then the jets will coalesce and destroy the forming CVP vortical structure, also reducing mixing performance. Assume that the spacing between jets is optimal given

$$
S=r d=J^{1 / 2} d
$$

Equation B3 may then be combined with equation B4 to form

$$
\sqrt{J_{1}}\left(\frac{S_{1}}{D}\right)=\sqrt{J_{2}}\left(\frac{S_{2}}{D}\right)
$$

Equation B5 is equivalent to the scaling law suggested by Holdeman [21]. Now consider the shape of the jets when viewed from the axial direction. Figure B2 illustrates how a jet spreads not only laterally but also radially within the pipe. Assume several jets were to penetrate to a distance $R_{1 / 2}$ that splits the flow area equally into a center circle and a torus. In a simplified case where the jet remains round, roughly half of the jet would be available to interact with crossflow fluid on the interior and the remainder would be available to interact with crossflow fluid on the periphery. 


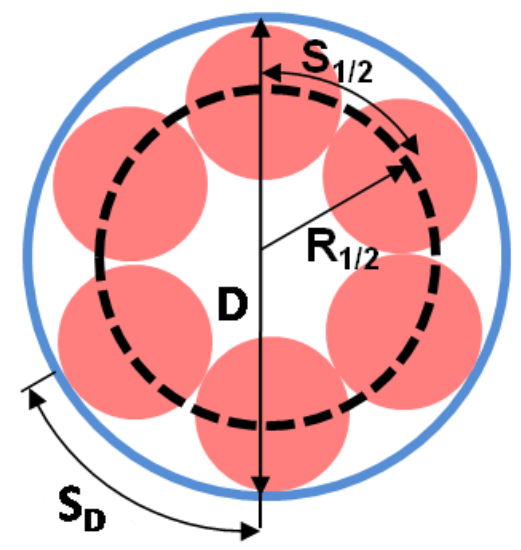

Figure B2: Jet distribution within a pipe.

Holdeman scaling comes from two arguments of developing jets. First, the size of the jet should be approximately $r d$ as they begin to touch. Second, the jets must have enough momentum relative to the crossflow to penetrate to this $R_{1 / 2}$ radius (corresponding to $r^{2} d \sim D$ ). This first condition may not be possible when the spacing $S$ is greater than $R_{1 / 2}$. This leads to the critical condition where $S=R_{1 / 2}$ and using the geometric identity $S=r \theta$, it follows that

$$
\frac{S_{1 / 2}}{R_{1 / 2}}=\frac{2 \pi}{n}=1 .
$$

This suggests that for values of $n<2 \pi$, we find that that too much space exists between the jets to merge effectively. Since jets are integer values, six jets most closely approximates this and should theoretically provide the best mixing irrespective of jet diameter and velocity.

\section{Appendix C: Complete Schematic for Flow Loop Facility}

Please refer to the following page for fold-out piping schematic. 


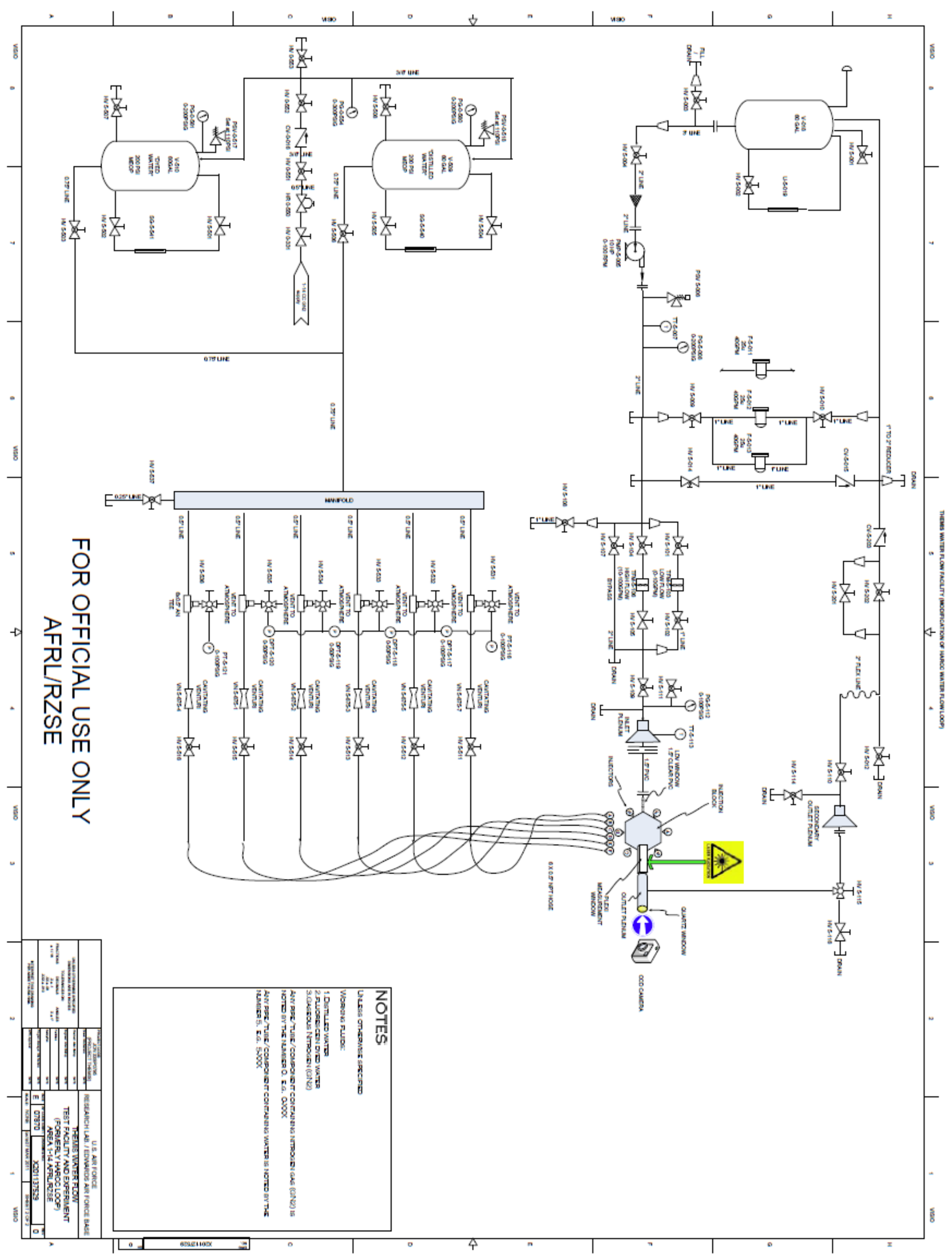




\section{Appendix D: Uncertainty Analysis}

The primary figure of merit is the unmixedness, $U$, is given by

$$
U=\frac{\sigma}{\bar{Z}(1-\bar{Z})}
$$

where $\sigma$ is the standard deviation of a spatial data field of time-averaged data and $\bar{Z}$ is the mean mixture fraction as a function of the relative flow rates. Sigma may be expressed as

$$
\sigma=\sqrt{\frac{\sum_{i=1}^{N}\left(C_{i}-\bar{C}\right)^{2}}{(N-1)}}
$$

where $N$ is the number of samples, $C_{i}$ is the mixture fraction at each pixel, $\bar{C}$ is the mean of the set $C_{i}$ expressed as

$$
\bar{C}=\sum_{i=1}^{N} C_{i} / N
$$

The mean mixture fraction $\bar{Z}$ is given by

$$
\bar{Z}=\frac{n Q_{j}}{n Q_{j}+Q_{c}}
$$

where $n$ is the number of jets, $Q_{j}$ is the flowrate of a single jet and $Q_{c}$ is the flowrate of the crossflow.

The equation for unmixedness may be differentiated to find the RMS error for a given test condition. This yields

$$
d U=\sqrt{\left[\left(\frac{\partial U}{\partial \sigma}\right) d \sigma\right]^{2}+\left[\left(\frac{\partial U}{\partial \bar{Z}}\right) d \bar{Z}\right]^{2}}
$$

By taking the partial differentials of $U$ with respect to $\sigma$ and $\bar{Z}$, one obtains respectively

$$
\left(\frac{\partial U}{\partial \sigma}\right)=\frac{1}{\bar{Z}(1-\bar{Z})}
$$

and

$$
\left(\frac{\partial U}{\partial \bar{Z}}\right)=\frac{(2 \bar{Z}-1) \sigma}{[\bar{Z}(1-\bar{Z})]^{2}}
$$


The differential $d \sigma$ may expressed as the total differential of its constituents such that

$$
d \sigma=\sqrt{\left[\left(\frac{\partial \sigma}{\partial C_{i}}\right) d C_{i}\right]^{2}+\left[\left(\frac{\partial \sigma}{\partial \bar{C}}\right) d \bar{C}\right]^{2}}
$$

In this experiment, the value $C_{i}$ was determined by calibrating each pixel intensity to a known bulk mixture fraction. Each pixel can be described by a simple linear equation such that

$$
C_{i}=m_{C_{i}} I+b_{C_{i}}
$$

where $m_{C_{i}}$ and $b_{C_{i}}$ are the calibration coefficients and $I$ is the measured pixel intensity. The differential value $d C_{i}$ can be described by the equation

$$
d C_{i}=\left|\left(\frac{\partial C_{i}}{\partial I}\right) d I\right|=\left|m_{C_{i}} d I\right|
$$

The value of the differential pixel intensity $d I$ is simply the smallest bin available for data collection (also known as the least scale reading or LSR). On a 16-bit camera, this is equivalent to $1 / 2^{16}$ or $1 / 65536$.

The equation for mean pixel intensity $\bar{C}$ can be differentiated to yield

$$
d \bar{C}=\left|\left(\frac{\partial \bar{C}}{\partial C_{i}}\right) d C_{i}\right|=\left|\left(\frac{\partial \bar{C}}{\partial C_{i}}\right) m_{C_{i}} d I\right|
$$

The partial differential of $\bar{C}$ with respect to $C_{i}$ is simply the inverse of the number of samples. This yields

$$
d \bar{C}=\left|\left(\frac{1}{N}\right) m_{C_{i}} d I\right|
$$

For a given data image, $\mathrm{N}$ is on the order of 100,000 useful pixels. This makes $(1 / N)$ on the order of $10^{-5}$. The calibration process to determine an $m_{C_{i}}$ value for each pixel indicates that the values of these coefficients are on the order of $10^{-4}$. Combining this with the LSR value for the data camera, the total order of magnitude for $d \bar{C}$ is on the order of $10^{-13}$. The term for $d C_{i}$ does not include the $(1 / N)$ 
term, yielding a differential on the order of $10^{-8}$. The total statement of $d \sigma$ may now be given including the partial derivatives of $\sigma$ with respect to $C_{i}$ and $\bar{C}$ as

$$
d \sigma=\sqrt{\left[\frac{2\left(C_{i}-\bar{C}\right)}{(N-1)} m_{C_{i}} d I\right]^{2}+\left[\frac{2\left(\bar{C}-C_{i}\right)}{(n-1)}\left(\frac{1}{N}\right) m_{C_{i}} d I\right]^{2}} .
$$

The term for $d \bar{Z}$ must now be addressed by expanding its differential terms such that

$$
d \bar{Z}=\sqrt{\left[\left(\frac{\partial \bar{Z}}{\partial n}\right) d n\right]^{2}+\left[\left(\frac{\partial \bar{Z}}{\partial Q_{j}}\right) d Q_{j}\right]^{2}+\left[\left(\frac{\partial \bar{Z}}{\partial Q_{j}}\right) d Q_{c}\right]^{2}}
$$

where the partial derivatives of $\bar{Z}$ are

$$
\begin{aligned}
\left(\frac{\partial \bar{Z}}{\partial n}\right) & =\frac{Q_{j} Q_{c}}{\left(n Q_{j}+Q_{c}\right)^{2}} \\
\left(\frac{\partial \bar{Z}}{\partial Q_{j}}\right) & =\frac{n Q_{c}}{\left(n Q_{j}+Q_{c}\right)^{2}}
\end{aligned}
$$

and

$$
\left(\frac{\partial \bar{Z}}{\partial Q_{c}}\right)=\frac{-n Q_{j}}{\left(n Q_{j}+Q_{c}\right)^{2}}
$$

Since $n$ is an integer value that is precisely controlled by the test conditions, its differential value $d n$ must be zero. This negates the first term.

The flow rate of a single jet is determined by a cavitating venturi. Although the theory behind a cavitating venturi will not be discussed here, after calibration the flow rate of a single jet may be expressed solely as a function of the inlet pressure, such that

$$
Q_{j}=m_{Q_{j}} P_{i n}+b_{Q_{j}}
$$

and the differential jet flow rate is

$$
d Q_{j}=\left|\left(\frac{\partial Q_{j}}{\partial P_{i n}}\right) d P_{i n}\right|=\left|m_{Q_{j}} d P_{i n}\right|
$$


The inlet pressure is measured by pressure transducers that output a measured voltage. The calibration equation for these transducers is

$$
P_{\text {in }}=m_{P_{\text {in }}} V+b_{P_{\text {in }}}
$$

and the corresponding differential pressure is

$$
d P_{\text {in }}=\left|\left(\frac{\partial P_{\text {in }}}{\partial V}\right) d V\right|=\left|m_{P_{\text {in }}} d V\right|
$$

The differential voltage $d V$ is the least scale reading of the output voltages, equal to $1 \mathrm{mV}$. For $d Q_{j}$ this yields

$$
d Q_{j}=\left|m_{Q_{j}} m_{P_{i n}} d V\right|
$$

The flowmeter for the primary flow is calculated in a different manner. The turbine flowmeter outputs an alternating current (and consequently voltage) with a frequency calibrated to a particular flowrate. The equation for this primary flow rate is

$$
Q_{c}=m_{Q_{c}} f+b_{Q_{c}}
$$

where $f$ is the frequency. The expression for the differential flowrate is consequently

$$
d Q_{c}=\left|\left(\frac{\partial Q_{c}}{\partial f}\right) d f\right|=\left|m_{Q_{c}} d f\right|
$$

In the calibration process, the frequency is transformed via fast Fourier transform from the measured voltages in the data acquisition system. This relationship is also linear, such that

$$
f=m_{f} V+b_{f}
$$

The differential $d f$ is then

$$
d f=\left|\left(\frac{\partial f}{\partial V}\right) d V\right|=\left|m_{f} d V\right|
$$


where $d V$ is again the LSR value for voltage, equal to $1 \mathrm{mV}$. The differential primary flowrate can then be expressed as

$$
d Q_{c}=\left|\left(\frac{\partial Q_{c}}{\partial f}\right) d f\right|=\left|m_{Q_{c}} m_{f} d V\right|
$$

All calibration coefficients were determined over a slightly larger range of operating conditions than those actually used in the test campaign, to identify any non-linearities in the behavior of the measurement devices. The linear $\mathrm{R}^{2}$ values were determined to be satisfactory for all devices $\left(\mathrm{R}^{2}>\right.$ $0.995)$.

The differential value $d \bar{Z}$ may now be expressed in terms of measureable quantities, such that

$$
d \bar{Z}=\sqrt{\left[\left(\frac{n Q_{c}}{\left(n Q_{j}+Q_{c}\right)^{2}}\right) m_{Q_{j}} m_{P_{i n}} d V\right]^{2}+\left[\left(\frac{-n Q_{j}}{\left(n Q_{j}+Q_{c}\right)^{2}}\right) m_{Q_{c}} m_{f} d V\right]^{2}} .
$$

A sample calculation is now presented for the condition expected to propagate the greatest relative error. The test conditions are:

- $n=6$

- $Q_{j}=0.7$

- $Q_{c}=17.057$

For reference, the total unmixedness error $d U$ is

$$
d U=\sqrt{\left[\left(\frac{\partial U}{\partial \sigma}\right) d \sigma\right]^{2}+\left[\left(\frac{\partial U}{\partial \bar{Z}}\right) d \bar{Z}\right]^{2}}
$$

Based on the flow rates, $\bar{Z}=0.1976$. The first partial differential is then

$$
\left(\frac{\partial U}{\partial \sigma}\right)=\frac{1}{\bar{Z}(1-\bar{Z})}=\frac{1}{0.1976(1-0.1976)}=6.3074
$$


The term $d \sigma$ is

$$
d \sigma=\sqrt{\left[\frac{2\left(C_{i}-\bar{C}\right)}{(N-1)} m_{C_{i}} d I\right]^{2}+\left[\frac{2\left(\bar{C}-C_{i}\right)}{(n-1)}\left(\frac{1}{N}\right) m_{C_{i}} d I\right]^{2}} .
$$

Given a normal distribution of a random set of data, a 95\% confidence interval incorporates data points within $\pm 2 \sigma$. Therefore, for this case it is appropriate to calculate the value for $\bar{C}$ within the analysis region and select a value for $C_{i}$ on the edge of this confidence interval. This value for sigma is approximately 0.0152 . The count $N$ for this data set is 147467 . The value for $m_{C_{i}}$ is pixel dependent, but for nearly the entire analysis region is approximately $4 \mathrm{E}-04$. The value of $d I$ for all cases is $1 / 65536$. This yields a value of $d \sigma$ to be approximately 1.26E-15. For the first $d U$ term, this then yields

$$
\left|\left(\frac{\partial U}{\partial \sigma}\right) d \sigma\right|=\left|(6.3074) 1.26 * 10^{-15}\right|=7.936 * 10^{-15}
$$

This value is sufficiently small that it may be neglected for the purpose of this thesis. The second partial derivative in the $d U$ equation is

$$
\left(\frac{\partial U}{\partial \bar{Z}}\right)=\frac{(2 \bar{Z}-1) \sigma}{[\bar{Z}(1-\bar{Z})]^{2}}
$$

Incorporating $\bar{Z}=0.1976$ and $\sigma=0.0076$, this partial derivative has a value of

$$
\left(\frac{\partial U}{\partial \bar{Z}}\right)=\frac{(2 * 0.1976-1) 0.0076}{[0.1976(1-0.1976)]^{2}}=-0.182839
$$

Recalling the equation for the differential $d \bar{Z}$ to be

$$
d \bar{Z}=\sqrt{\left[\left(\frac{n Q_{c}}{\left(n Q_{j}+Q_{c}\right)^{2}}\right) m_{Q_{j}} m_{P_{i n}} d V\right]^{2}+\left[\left(\frac{-n Q_{j}}{\left(n Q_{j}+Q_{c}\right)^{2}}\right) m_{Q_{c}} m_{f} d V\right]^{2}} .
$$

Plugging in test conditions and calibration coefficients yields 


$$
d \bar{Z}=\sqrt{\begin{array}{l}
{\left[\left(\frac{6 * 17.057}{(6 * 0.7+17.057)^{2}}\right) 0.00378 * 0.003368 * 1\right]^{2}} \\
+\left[\left(\frac{-6 * 0.7}{(6 * 0.7+17.057)^{2}}\right) 0.0277 * 0.43778 * 1\right]^{2}
\end{array}}=1.1275 * 10^{-4}
$$

Therefore, after negation of the $d \sigma$ term, the total differential of unmixedness is

$$
d U=\left|\left(\frac{\partial U}{\partial \bar{Z}}\right) d \bar{Z}\right|=\left|-0.182839 * 1.1275 * 10^{-4}\right|=2.0615 * 10^{-5}
$$

The total error in unmixedness can then be expressed as

$$
\frac{d U}{U}=\frac{2.1136 * 10^{-5}}{0.01921}=0.00107=0.107 \%
$$

This low value for $d U / U$ is based upon a zeroth order analysis and does not take several factors into consideration. The first of these is the error associated with the analysis code. Depending upon the sample size (see Section 5.1 for more detail), this error is approximately $4 \%$ for the size of images analyzed. Additionally, some random error does exist in the system, including but not limited to: unsteadiness of the pump, unsteadiness of the turbine flowmeter and the turbulent mixing processes of the jets themselves. When the three repeat points for the single jet case $(B(d / D) \cong 0.5,0.75,1.0)$ are analyzed heuristically such that their difference of each pair of data points is divided by their average, the values range from $4.65 \%$ to $0.89 \%$. These calculated values for unmixedness take all of these errors into account and suggest that the cap for error in any one unmixedness measurement is on the order of $5 \%$. 